\title{
Microalgal Heterotrophic and Mixotrophic Culturing for Bio-refining: From Metabolic Routes to Techno-economics
}

\author{
Octavio Perez-Garcia and Yoav Bashan
}

\begin{abstract}
In comparison with conventional photo-autotrophic cultivation, heterotrophic and mixotrophic cultivations of microalgae offers a feasible strategy to produce biomass and valuable chemicals through biorefinery processes. Supplementing microalgae cultures with organic carbon sources increase the biomass production and lipid/carbohydrate contents in cells. Consequently, this yields high productivity of biorefined products such as biodiesel, ethanol, starch and polyunsaturated fatty acids. Nevertheless, the addition of an organic carbon source imposes the necessity to pre-produce them and increase the cost and susceptibility of the cultures to microbial contamination. This chapter reviews the aspects related to the heterotrophic and mixotrophic cultivation of microalgae. These include advantages and limitations, metabolic routes of organic carbon assimilation, alternative carbon sources and main considerations for cultivation systems. It provides a comprehensive review of cultivated species, organic carbon sources and recently achieved productivity metrics. The chapter includes discussions regarding the main commercial products obtained using these cultivation modes and a simplified techno-economic analysis of the full biorefinery operation. The overarching aim of the chapter was to depict the main challenges for commercialization of chemical products using heterotrophic/mixotrophic cultivation of microalgae and identifies the promising research lines to achieve the same.
\end{abstract}

Keywords Heterotrophic microalgae - Mixotrophic microalgae - Metabolism • Microalgae cultivation $\bullet$ Bioreactor $\bullet$ Organic carbon source $\bullet$ Biorefinery $\bullet$ Technoeconomics $\bullet$ Biofuels $\bullet$ Valuable chemicals

\footnotetext{
O. Perez-Garcia

Department of Civil and Environmental Engineering, The University of Auckland, 20 Symonds Street, CBD, Auckland 1010, New Zealand

e-mail: rper079@aucklanduni.ac.nz; octavio.perez@auckland.ac.nz

Y. Bashan $(\bowtie)$

Environmental Microbiology Group, Northwestern Center for Biological Research (CIBNOR), Calle IPN 195, La Paz, B.C.S. 23096, Mexico
}

The Bashan Institute of Science, 1730 Post Oak Ct., Auburn, AL 36830, USA

Department of Entomology and Plant Pathology, Auburn University,

209 Life Sciences Building, Auburn, AL 36849, USA

e-mail: ybb0001@auburn.edu; ybashan@ cibnor.mx 


\section{Introduction}

Algae, in particular microalgae, are one of the most promising feedstock for sustainable production of biofuels and valuable chemicals. Nowadays, food supplements from microalgae comprise an important market in which compounds, such as $\beta$-carotene, astaxanthin, and polyunsaturated fatty acids (PUFAs), such as eicosapentaenoic acid (EPA), docosahexaenoic acid (DHA), and polysaccharides, such as $\beta$-glucan, dominate (Hudek et al. 2014). Research on algae is not only focusing on improving production of products, but also new algae products, such as biodiesel, bio-ethanol, and renewable chemicals, such as starch, sucrose and ethylene. In every case, the raw material for bio-refining these compounds is microalgae biomass, which is produced by culturing microalgae under controlled or semi-controlled conditions.

To achieve the largest possible microalgal productivity in a cost-effective way, selection of a microalgae mode of cultivation is of vital importance. Four major modes of microalgae cultivation can be adopted, namely photo-autotrophic, heterotrophic, photo-heterotrophic, and mixotrophic (Table 1; Wang et al. 2014). In general, microalgae are commonly grown by fixing dissolved, inorganic carbon $\left(\mathrm{CO}_{2}\right)$ and absorbing solar energy. Therefore, like most land-based plants, they perform photosynthesis and are photo-autotrophs. At the same time, some species of microalgae are also heterotrophic, using organic compounds in the growth medium as carbon and energy sources; therefore, they do not need light as an energy source (Chen 1996).

Heterotrophic growth is an aerobic process where assimilation of organic substrates generates energy through oxidative phosphorylation accompanied by oxygen consumption as the final electron acceptor. Mixotrophic cultivation is the growth mode where microalgae simultaneously uses inorganic $\mathrm{CO}_{2}$ and organic carbon sources in the presence of light (Kang et al. 2004); therefore, photo-autotrophy and heterotrophy occur simultaneously (Wang et al. 2014). $\mathrm{CO}_{2}$ is fixed through photosynthesis, which is influenced by illumination, while organic compounds are assimilated through aerobic respiration, which is affected by the availability of organic carbon. Several species are able to switch between photo-autotrophic and heterotrophic growth. This should not be confused with the mixotrophy regime, where both ways of uptake (organic and inorganic) occur at the same time. Mixotrophic microalgae use different sources of energy and carbon,

Table 1 Growth modes of algae (microalgae) cultivation

\begin{tabular}{l|l|l|l|l}
\hline Growth mode & $\begin{array}{l}\text { Energy } \\
\text { source }\end{array}$ & $\begin{array}{l}\text { Carbon } \\
\text { source }\end{array}$ & $\begin{array}{l}\text { Light availability } \\
\text { requirements }\end{array}$ & $\begin{array}{l}\text { Metabolism } \\
\text { variability }\end{array}$ \\
\hline Photo-autotrophic & Light & Inorganic & Obligatory & $\begin{array}{l}\text { No switch between } \\
\text { sources }\end{array}$ \\
\hline Heterotrophic & Organic & Organic & No requirements & $\begin{array}{l}\text { Switch between } \\
\text { sources }\end{array}$ \\
\hline Photoheterotrophic & Light & Organic & Obligatory & $\begin{array}{l}\text { Switch between } \\
\text { sources }\end{array}$ \\
\hline Mixotrophic & $\begin{array}{l}\text { Light and } \\
\text { organic }\end{array}$ & $\begin{array}{l}\text { Inorganic and } \\
\text { organic }\end{array}$ & No obligatory & $\begin{array}{l}\text { Simultaneous } \\
\text { utilization }\end{array}$ \\
\hline
\end{tabular}


they may use organic or inorganic sources and light in different combinations. Mixotrophy makes microalgae more flexible because it may gather both carbon and energy demand by organic or inorganic sources and light simultaneously (Chen et al. 2011). In photo-heterotrophy, growth cells use light for energy, fix fixation of nitrogen and organic matter as a carbon source without $\mathrm{CO}_{2}$ (Chen et al. 2011). Hence, because organic carbon and light are compulsory for photo-heterotrophic cultivation, it is rarely used as an approach to produce microalgal biomass to process valuable compounds (Wang et al. 2014). Therefore, photo-heterotrophic cultivation will not be discussed further.

Heterotrophic and mixotrophic cultivation of microalgae can be used in biorefinery processes. It is important to have a clear picture of where the cultivation regime fits in the overall process. Microalgae cultivation is the sum of procedures and techniques to produce the feedstock biomass that subsequently is refined to obtain valuable products. Therefore, prospecting, selecting, or developing a strain (by genetic and metabolic engineering) together with microalgae cultivation, and growth medium preparation belong to the "upstream" part of the entire process of biomass harvesting, de-watering, extraction of valuable compounds, transformation and purification belong to the "downstream" part of the process. Figure 1 presents an overview of the complete biorefinery process and highlights where the microalgae cultivation step fits in. Heterotrophic and mixotrophic cultivation of algae requires a supply of organic carbon that is provided from chemicals, such as glucose or acetate, crop flours, wastewater, food and milk industrial wastes, and lignocellulosic materials. Water supplemented with organic carbon and inorganic nutrients, such as nitrogen and phosphorus, are used to prepare the growth medium for cultivation. Medium preparation involves filtration, mixing, diluting, sterilizing, and hydrolysis of carbon sources. Appropriate strain selection supplies the inoculum (microalgae seed culture) for microalgae biomass production. Strain selection involves two main approaches: bio-prospecting and isolating strains with desirable metabolic and physiological capabilities and/or developing strains with capabilities by metabolic and genetic engineering. The cultivation step (biomass production) can be achieved by photo-autotrophic, heterotrophic, or mixotrophic growth of microalgae. The culture can be operated as batch, fed-batch, continuous, or semi-continuous modes and can be at a laboratory, pilot, or commercial scale. After cultivation, various downstream sub-processes follow to obtain the desired chemical products from the biomass. In general, these sub-processes include: (1) biomass harvesting, (2) extraction of the potential valuable chemicals, (3) separation of various microalgae raw chemicals extracted simultaneously, (4) transformation of raw materials to useful products, and (5) final purification for commercial products.

Compared with common photo-autotrophic microalgal growth, heterotrophic and mixotrophic approaches have the potential to provide larger biomass and yield of valuable organic compounds. These cultivation modes have their own technical challenges that impede large scale cultivation for producing valuable chemicals, including biofuels, in a cost-effective way (Bassi et al. 2014; Liang 2013; PerezGarcia et al. 2011a). This chapter discusses the advantages and challenges, as well as the following topics: key aspects of cell metabolism related to growth modes, 


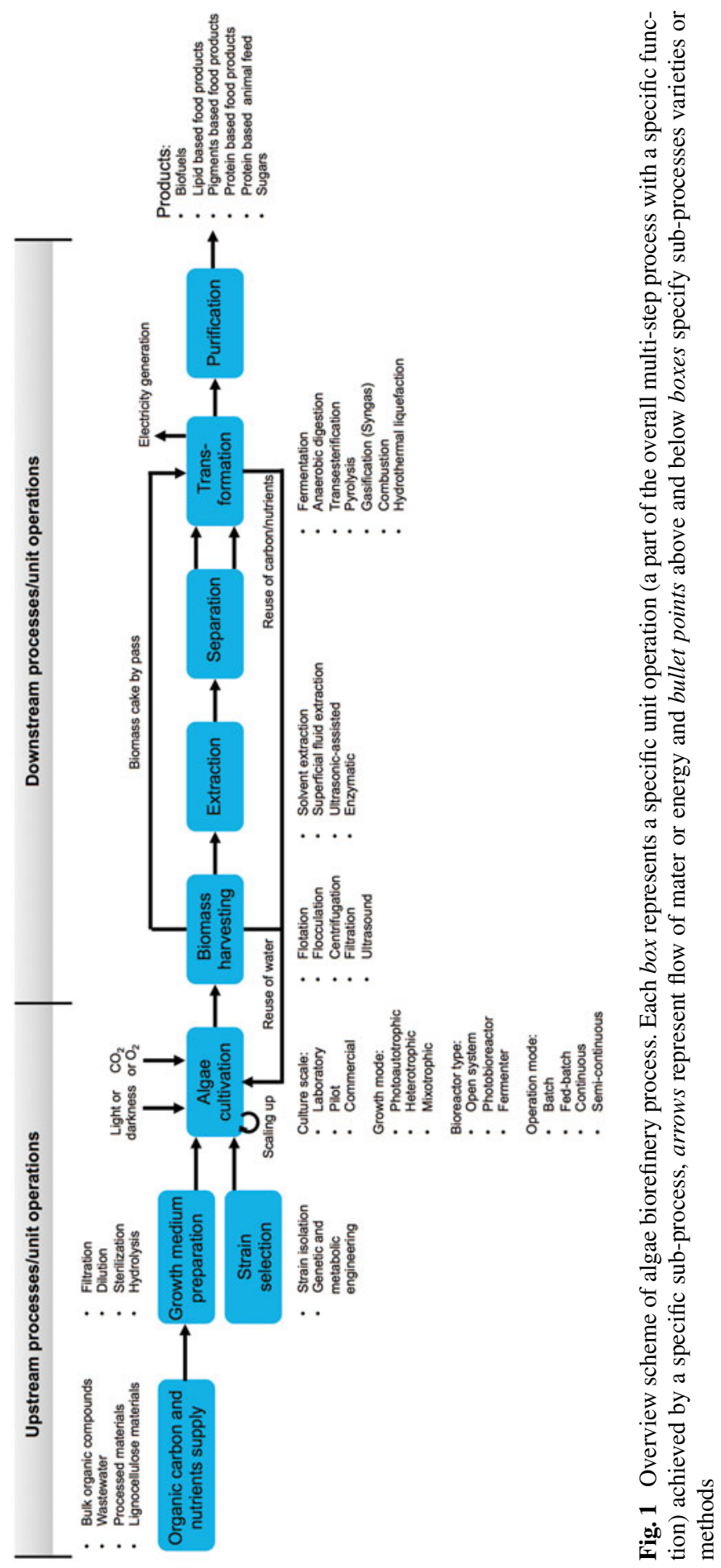


potential carbon sources, and important aspects of cultivation methods. Finally, we present an analysis of important techno-economic aspects regarding heterotrophic and mixotrophic cultivation of microalgae and highlight opportunities to increase economic feasibility and sustainability of these technologies.

\section{Advantages and Limitations of Heterotrophic and Mixotrophic Cultivation of Microalgae}

\subsection{Advantages}

The advantages of heterotrophic cultivation of microalgae, in comparison with photo-autotrophic cultivation are the following: (a) Higher growth rate and biomass density (also called biomass productivity); (b) Higher lipid content per dry weight of cells (lipid productivity); (c) Higher biomass productivity per area of culture; (d) Cheaper and simpler bio-reactor design; (e) Easier scaling-up process; (f) The possibility to manipulate biomass composition by changing the culture medium's organic substrate that stimulates specific metabolic and biosynthetic pathways; and (g) Potential to remove organic carbon and several types of nitrogen and phosphorus compounds from wastewater (Brennan and Owende 2010; Chen 1996; Li et al. 2007; Lu et al. 2010; Miao and Wu 2006; Ogbonna et al. 2000; Xiong et al. 2010; Perez-Garcia et al. 2010).

Mixotrophic growth offers several advantages: (a) Higher growth rates than either heterotrophic and photo-autotrophic regimes by shortening growth cycles and producing higher biomass; (b) Prolonged exponential growth phase; (c) Reduction of lost biomass from respiration during dark hours; (d) Reduction or stopping of photo-inhibitory effect; (e) Flexibility to switch the cultivation regime to heterotrophic or photo-autotrophic regimens at will; and (f) Protection from photo-oxidative damage stimulated by accumulating oxygen in enclosed photo-bioreactors (Chojnacka and Marquez-Rocha 2004; Chojnacka and Noworyta 2004; Kröger and Müller-Langer 2011; Vonshak et al. 2000; Wang et al. 2014).

Production of microalgae under heterotrophic cultivation is a very successful route to commercialization of high-value chemicals, such as pharmaceuticals and food supplements. Economic advantages of heterotrophic and mixotrophic growth over the photo-autotrophic growth when mass-producing microalgae was summarized over 15 years ago by Chen (1996) and Borowitzka (1999); they are still valid. These microalgae biotechnology pioneers recognized that high cell population and biomass densities (between 20 and $100 \mathrm{~g} \mathrm{~L}^{-1}$ ) can be achieved under heterotrophic cultivation in fermenters in complete darkness. Compared to the photo-autotrophic condition, heterotrophic conditions have enhanced concentrations of Chlorella protothecoides up to 3.4 times (Shi et al. 2002), of C. vulgaris up 
to 4.8 times (Liang et al. 2009; Perez-Garcia et al. 2010; Choix et al. 2012a;), and of $C$. sorokiniana up to 3.3 times (Zheng et al. 2012). A range of biomass between 4 and $20 \mathrm{~g} \mathrm{~L}^{-1}$ day $^{-1}$ of microalgae are commonly produced by using heterotrophic cultivation (Graverholt and Eriksen 2007; Li et al. 2007; Shen et al. 2010; Xiong et al. 2010). This compares with $0.06-0.1 \mathrm{~g} \mathrm{~L}^{-1} \mathrm{day}^{-1}$ in open cultivation ponds and $0.36 \mathrm{~g} \mathrm{~L}^{-1}$ day $^{-1}$ in closed photo-bioreactors (Pulz 2001). Using heterotrophic and mixotrophic cultivation, researchers successfully increased the lipid content, compared to autotrophic cultivation. For example, the lipid content in a heterotrophic culture of $C$. protothecoides is $55.2 \%$, whereas an autotrophic culture of the same microalgae provides about $15 \%$ (Xu et al. 2006). Cultivation of $C$. vulgaris under heterotrophic conditions, without nitrogen deprivation yielded faster growth and accumulated more lipids than under autotrophic conditions (Leyva et al. 2014).

Elimination of light in heterotrophic cultures reduces bioreactor construction costs and allows better control of growth of the microalgae and minimizes contamination by photosynthetic microorganisms. The design of photo-bioreactors for photo-autotrophic cultivation maximizes the area exposed to light irradiation to provide microalgae cells optimum photons for photosynthesis (Molina Grima et al. 1999; Rodolfi et al. 2009). While this is technologically feasible, it is difficult to reach a high biomass density of microalgae in photo-bioreactors. This happens because penetration of light in the medium is inversely proportional to cell concentration. Mutual shading of cells causes less light in the inner parts of the bioreactor. When this happens, production of biomass is very low; hence, there are very low yields of products (Borowitzka 1999; Chen et al. 2011; Molina Grima et al. 1999). Low biomass density in a reactor also increases the biomass harvesting cost. As a result, producing valuable products, specifically biofuels, from photo-autotrophic microalgae needs a long development time and huge investments before it becomes commercially viable (Liang 2013). For example, an insignificant amount of biodiesel is currently made from microalgae grown under photo-autotrophic conditions and this is not considered a commercial source. In shorter periods of time, developments to produce an abundant quantity of valuable products from microalgae should concentrate on cultivating microalgae in heterotrophic or mixotrophic growth modes, using cheap sugars or organic acids as carbon and energy sources.

Finally, cultivation of microalgae under heterotrophic and mixotrophic conditions provide environmental services. Efficiency of nutrient removal (N, P) from municipal, agricultural, or industrial wastewater by microalgae is higher under aerobic, dark heterotrophic conditions and mixotrophic conditions than under photo-autotrophic conditions (Andrade and Costa 2007; Li et al. 2011; Ogbonna et al. 2000; PerezGarcia et al. 2010, 2011b; Zhou et al. 2013). In addition, although it is not possible to feed heterotrophic microalgae with $\mathrm{CO}_{2}$ emissions, $\mathrm{CO}_{2}$ sequestration is included in the overall process cycle because the initial organic substrate is produced by photosynthetic plants (Brennan and Owende 2010). 


\subsection{Limitations}

While the metabolic and physiological features of heterotrophic and mixotrophic cultivation offer the possibility of greatly increasing cell density and productivity of microalgae, all these advantages notwithstanding, commercial production of cheap chemicals, mainly biofuel, using these cultivation regimes, are not presently cost effective (Kröger and Müller-Langer 2011; Liang 2013; Tabernero et al. 2012; Wang et al. 2014). This is caused by several major limitations: (a) Increasing energy expenses and general costs by adding an organic substrate; (b) Contamination and competition with other microorganism that grow faster than the microalgae; (c) Inability to produce light-induced metabolites into heterotrophic cultures; (d) Limited number of microalgal species that can grow heterotrophically and mixotrophycally; and (e) Indirect use of arable land for carbon source production reduces the main advantage that microalgal cultivation systems has over land-based crops (Borowitzka 1999; Chen 1996). Finally, it is important to acknowledge that heterotrophic or mixotrophic growth does not necessarily yield faster growth rates than photo-autotrophic cultivation.

Cultivation of heterotrophic and mixotrophic microalgal strains is based on highcost carbon sources. With the essential requirement of organic compounds, the long term operation cost of heterotrophic cultivation is higher than for photo-autotrophic cultivation, even after reducing the cost of illumination in the latter case (Tabernero et al. 2012). For example, lipids from heterotrophic microalgae can partly replace fossil oils in biodiesel production, but from an economic standpoint, this concept is restricted by the costs of nutrients. For example, $80 \%$ of the costs for producing biodiesel from Chlorella protothecoides comes from glucose in the growth medium (Li et al. 2007), Glucose as the carbon source yields the most lipids for many microalgae (Perez-Garcia et al. 2011a, b). Additionally, costs for nitrogen, phosphorus, vitamins, and trace metals are additional. The theoretical efficiency of heterotrophic microalgae feed with glucose is up to $75 \%$ in the conversion to biodiesel. However, since glucose or other organic nutrients also have to be produced, in most cases from land-based crops, the efficiency of this organic nutrient production has to be added to the overall efficiency of heterotrophic or mixotrophic cultivation (Kröger and Müller-Langer 2011).

Another major disadvantage of heterotrophic and mixotrophic cultivation is their vulnerability to contamination by other microorganisms, many of them are fast growers. This may reduce the quality and quantity of products of interest. Therefore, managing axenic monoalgal culture is of vital importance for cultivation. This increases the capital and operation costs of bioreactors (Wang et al. 2014; Yan et al. 2011). Heterotrophic microalgal growth requires an oxygen supply, mixing, and nutrients, all under sterile environment. For most studies, a stirred-tank bioreactor commonly known as a fermenter is adopted. At the laboratory or pilot scale, such bioreactors are suitable. However, these fermenters are extremely expensive and economically unrealistic for production of cheap biofuels and chemicals at an industrial scale because the required volume, sterilization devices, and power con- 
sumption for mixing and aeration considerably increase capital investment and operation costs (Liang 2013). Tabernero et al. (2012) comprehensively evaluated the techno-economic feasibility of biodiesel production at the industrial scale from heterotrophic microalgae by analyzing data related to production plant construction and operation costs and lipid productivities of $C$. protothecoides in heterotrophic cultures. A non-conservative analysis revealed that an industrial plant with production capacity of $10,000 \mathrm{t} \mathrm{year}^{-1}$ of biodiesel is not economically viable unless biomass residues were refined into proteins and carbohydrates and sold. A conservative estimate showed no viability of the plant even if the residues were sold. The main economic drawback comes from the large number and volume of bioreactors (up to 465 reactors, each with working volume of $150 \mathrm{~m}^{3}$ ) required to reach the targeted yearly production (Tabernero et al. 2012). Regardless of this recent grim assessment, mixotrophic cultivation should be further investigated for mass production of microalgae because it combines the features of photo-autotrophic growth, enhancing it by the partial use of organic nutrients for production of other high value chemicals (Kröger and Müller-Langer 2011).

Heterotrophic cultivation is inappropriate for most microalgae because most species are obligate autotrophs rather than facultative heterotrophs (Behrens 2005). Yet, some species are effectively grown in complete darkness and can be cultivated in conventional dark fermenters. The genera reported to grow heterotrophically include: Amphora, Ankistrodesmus, Chlamydomonas, Chlorella, Chlorococcum, Crypthecodinium, Cyclotella, Dunaliella, Euglena, Nannochloropsis, Nitzschia, Ochromonas, and Tetraselmis (Behrens 2005; Geider and Osborne 1989). Only a few microalgae species can grow mixotrophically. These include the freshwater Brachiomonas submarina, Chlorella spp., Chlorococcum sp., Cyclotella cryptica, Euglena gracilis, Haematococcus pluvialis, Nannochloropsis spp., Navicula saprophila, Nitzschia sp., Ochromonas minima, Phaeodactylum tricornutum, Rhodomonas reticulate and Scenedesmus obliquus (Bassi et al. 2014; Liang et al. 2009). Additionally, the cyanobacteria genera Anabaena, Spirulina and Synechococcus can be grown heterotrophically and mixotrophically (Chen et al. 1996; Kang et al. 2004). One strain of Chlorella protothecoides can grow on glucose, acetate, and other organic compounds in the dark, but it is unknown whether it can grow on organic carbon sources in the presence of light (Xu et al. 2006). It is important to consider that even though some species can grow on multiple carbon sources, not every microalgal species can be fed successfully with every organic nutrient for biomass production. For example, $C$. vulgaris yielded different biomass productivity in heterotrophic cultures from different carbon sources (Perez-Garcia et al. 2011a). Consequently a specific organic carbon source must fit with a specific microalga to obtain optimal growth or productivity of a compound.

To overcome the cost hurdle and make biorefineries of microalgae economically feasible, three main research areas need to be explored (Kröger and Müller-Langer 2011; Liang 2013; Tabernero et al. 2012): (a) Finding a low-value or, even better, a zero-value carbon source to support heterotrophic/mixotrophic microalgal growth; (b) Design economical bioreactors that are appropriate for industrial scale of heterotrophic/mixotrophic microalgae; and (c) Reduce downstream processing costs, especially those of harvesting and biomass transformation, for example, transesteri- 
Table 2 Challenges and opportunities for the heterotrophic/mixotrophic cultivation of microalgae

\begin{tabular}{|c|c|}
\hline Limitation & Opportunities \\
\hline \multirow[t]{4}{*}{ Carbon sources costs } & $\begin{array}{l}\text { Investigate new sources of cheap organic carbon, such as } \\
\text { wastewaters, lignocellulosic material, and industrial } \\
\text { processes waste }\end{array}$ \\
\hline & $\begin{array}{l}\text { Bio-prospection of strains able to assimilate cheap carbon } \\
\text { sources }\end{array}$ \\
\hline & $\begin{array}{l}\text { Metabolic engineering of strains able to assimilate cheap } \\
\text { carbon sources }\end{array}$ \\
\hline & Improve methods for breakdown of lignocellulose material \\
\hline \multirow{5}{*}{$\begin{array}{l}\text { Competition by fast-growing } \\
\text { bacteria }\end{array}$} & Development of mixotrophic cultivation strategies \\
\hline & $\begin{array}{l}\text { Establishing cultures of microalgae able to thrive under } \\
\text { bacteria-adverse environmental conditions }\end{array}$ \\
\hline & Bio-prospection of fast-growing strains \\
\hline & Metabolic engineering of fast-growing strains \\
\hline & Immobilization of microalgae in polymers \\
\hline \multirow{6}{*}{$\begin{array}{l}\text { Bioreactor implementation and } \\
\text { operation costs }\end{array}$} & Cheaper materials for bioreactor vessel \\
\hline & $\begin{array}{l}\text { Implement alternative mixing strategies powered by a } \\
\text { renewable energy source (hydraulic or wind) }\end{array}$ \\
\hline & Implement cheap sterilization strategies \\
\hline & $\begin{array}{l}\text { Establish non-axenic microalgae cultures, such as open } \\
\text { ponds }\end{array}$ \\
\hline & $\begin{array}{l}\text { Increase productivity of the metabolites of interest by } \\
\text { optimizing bioreactor's operation parameters }\end{array}$ \\
\hline & $\begin{array}{l}\text { Risk assessment studies and regulations of GMOs in } \\
\text { large-scale facilities }\end{array}$ \\
\hline \multirow{5}{*}{$\begin{array}{l}\text { Downstream processes costs } \\
\text { (biomass harvesting and raw } \\
\text { product transformation) }\end{array}$} & $\begin{array}{l}\text { Enhance exo-polysaccharides production to promote } \\
\text { biomass flocculation }\end{array}$ \\
\hline & $\begin{array}{l}\text { Develop immobilization technique for the algae in } \\
\text { polymeric beads/sheets }\end{array}$ \\
\hline & Promote spontaneous excretion of metabolite of interest \\
\hline & Selection or design of strains that excrete products \\
\hline & $\begin{array}{l}\text { Avoid compound extraction and separation by directly } \\
\text { transform the biomass to products by pyrolysis, anaerobic } \\
\text { digestion, gasification. }\end{array}$ \\
\hline
\end{tabular}

fication in biodiesel. Table 2 summarizes the limitations involved in heterotrophic and mixotrophic cultivation of microalgae, as well as promising research and development opportunities to address these limitations.

\section{Heterotrophic and Mixotrophic Metabolism}

The mode of cultivation significantly influences the metabolism and growth pattern of microalgae and determines the quality and quantity of biorefinery products. Heterotrophic or mixotrophic metabolism does not mean that every 
microalgae species can ingest every organic compound dissolved in water. In principle, it is impossible to generalize the properties of heterotrophically grown microalgae in relation to certain organic compounds. The review of PerezGarcia et al. (2011b) provides an overview of the biochemistry behind use of nutrients in heterotrophic microalgae covering metabolism of major sources of carbon and nitrogen in heterotrophic and mixotrophic cultivation. The review summarizes the enzymatic pathways for assimilation of glucose, glycerol, and acetate, and the metabolism of nitrogen, including ammonium, nitrate, nitrite, urea, and organic nitrogen. This chapter, therefore, summarizes the main patterns with specific examples.

Table 4 presents metabolic performance data, such as maximum biomass concentration, growth rate, biomass productivity, and product productivity observed in studies on heterotrophic and mixotrophic growth of microalgae during mass cultivation. It shows that maximum growth rates for heterotrophic cultures for many microalgae range from 0.2 to $0.7 \mathrm{day}^{-1}$. Higher values, of up to $4.7 \mathrm{day}^{-1}$, have been reported for Schizochytrium mangrovei growing on food waste (Pleissner et al. 2013). Maximum growth rates observed in mixotrophic cultures ranged from 0.25 to 1.0 day $^{-1}$ and are, in general, higher than those reported for heterotrophic cultivation. In every study where heterotrophic and mixotrophic growth were compared under identical conditions (except light exposure), mixotrophic cultures had higher growth rate and higher biomass concentration at the end of the experiment (Table 4) (Cheirsilp and Torpee 2012; Li et al. 2011; Zhou et al. 2013). For instance, Chlorella spp. and Nannochloropsis spp. grown mixotrophycally on glucose yielded higher biomass concentration than heterotrophic and photo-autotrophic cultures (Cheirsilp and Torpee 2012). An interesting observation is that the highest growth rate under mixotrophic regime $\left(\mu_{m i x o}^{\max }\right)$ corresponds approximately to the sum of the maximum growth rates obtained under the photo-autotrophic and heterotrophic modes $\left(\mu_{\text {mixo }}^{\max }=\mu_{\text {photo }}^{\max }+\mu_{\text {hetero }}^{\max }\right)$ (Girard et al. 2014). Similar observations are known for other microalgae and cyanobacteria species, such as Chlorella regularis, C. vulgaris, Euglena gracilis, Haematococcus pluvialis, and Spirulina platensis (Ogbonna et al. 2002). Nevertheless, this is only a general rule; experimental verification of growth rate is required in each case.

Apart from cell metabolism, cell structure changes, depending on growth regime. Under a photo-autotrophic regime, transmission electron microscopy showed that chloroplasts were clearly visible in photosynthetic cells (Lebsky et al. 2001). Membranes were abundantly accumulated in these chloroplasts and a number of starch granules could also be seen. In contrast, thylakoid membranes rapidly disappeared within $48 \mathrm{~h}$ after cells had undergone heterotrophic metabolism, suggesting degeneration of chloroplasts. Instead, the cytoplasm was almost totally filled with large lipid droplets (de-Bashan et al. 2002a). Biochemical and ultra-structural experiments suggested that chlorophyll breakdown and chloroplast degeneration was associated with lipogenesis during the heterotrophic growth of $C$. protothecoides. These cells were initially grown under photo-autotrophic conditions and were rich of chloroplasts (Xiong et al. 2010). 


\subsection{Assimilation of Organic Carbon During Heterotrophic and Mixotrophic Cultivation}

\subsubsection{Glucose}

Glucose is the most commonly used carbon source for heterotrophic cultivation of microalgae, as is the case for many other microbial species. Far higher rates of growth and respiration are obtained with glucose than with any other substrate, such as other simple sugars, sugar alcohols, sugar phosphates, organic acids, and monohydric alcohols (Griffiths et al. 1960). This happens because glucose possesses more energy content per mol, compared with other substrates. For example, glucose produces $2.8 \mathrm{~kJ} \mathrm{~mol}^{-1}$ of energy compared to $0.8 \mathrm{~kJ} \mathrm{~mol}^{-1}$ for acetate (Boyle and Morgan 2009). The yield of $460 \mathrm{mg}$ algal biomass per gram of glucose was achieved with the cyanobacteria Spirulina platensis (Marquez et al. 1993). This yield is comparable to yeasts and other aerobic heterotrophs. Similar results were obtained with Chlorella protothecoides ( $\mathrm{Li}$ et al. 2007; Xu et al. 2006). The mass specific conversion rate from glucose to microalgal biomass varies from 40 to $64 \%$ (Li et al. 2007; $\mathrm{Xu}$ et al. 2006); whereas, the lipid yield conversion has a variation ranging from 19 to $31 \%$ of glucose (Kröger and Müller-Langer 2011).

Oxidative assimilation of glucose begins with phosphorylation of hexose, yielding glucose-6-phosphate, which is readily available for storage, cell synthesis, and respiration. An equivalent of a single phosphate bond is required per mole of glucose assimilated into glucose-6-phosphate. In that process, an additional 30 equivalents of phosphate bonds are generated by aerobic oxidation of a mole of glucose (Droop 1974). Of the several pathways used by microorganisms for aerobic glycolysis (breakdown of glucose), apparently only two, the Embden-Meyerhof pathway (EM pathway) and the Pentose Phosphate pathway (PP pathway) have been found in algae (Neilson and Lewin 1974). Under complete darkness heterotrophic growth, glucose is mainly metabolized via the PP pathway (Fig. 2), while the EM pathway is the main glycolytic process of cells in mixotrophic growth with light (Hong and Lee 2007; Lloyd 1974; Neilson and Lewin 1974; Yang et al. 2000). Both pathways are carried out in the cytosol and are functional in microalgae cells; however, the PP pathway might have a higher flux rate than the EM pathway, depending on light and presence of glucose.

The glycolytic EM pathway, Tricarboxylic Acid cycle (TCA cycle) and mitochondrial oxidative phosphorylation maintain high activities during photoautotrophic, heterotrophic, and mixotrophic growth of C. pyrenoidosa (chlorophyta) and Synechocystis sp (cyanobacteria) with glucose. This indicates a minor effect of the growth mode on these pathways in this species (Hong and Lee 2007; Yang et al. 2000). Central metabolic gene expression patterns (at the mRNA and protein levels) were analyzed for Synechocystis sp. growing under photo-autotrophic, heterotrophic, and mixotrophic conditions. The comparison showed that the majority of genes of the EM pathway and TCA cycle are expressed at the same levels under every trophic condition. However, expression of genes related to the Calvin cycle 


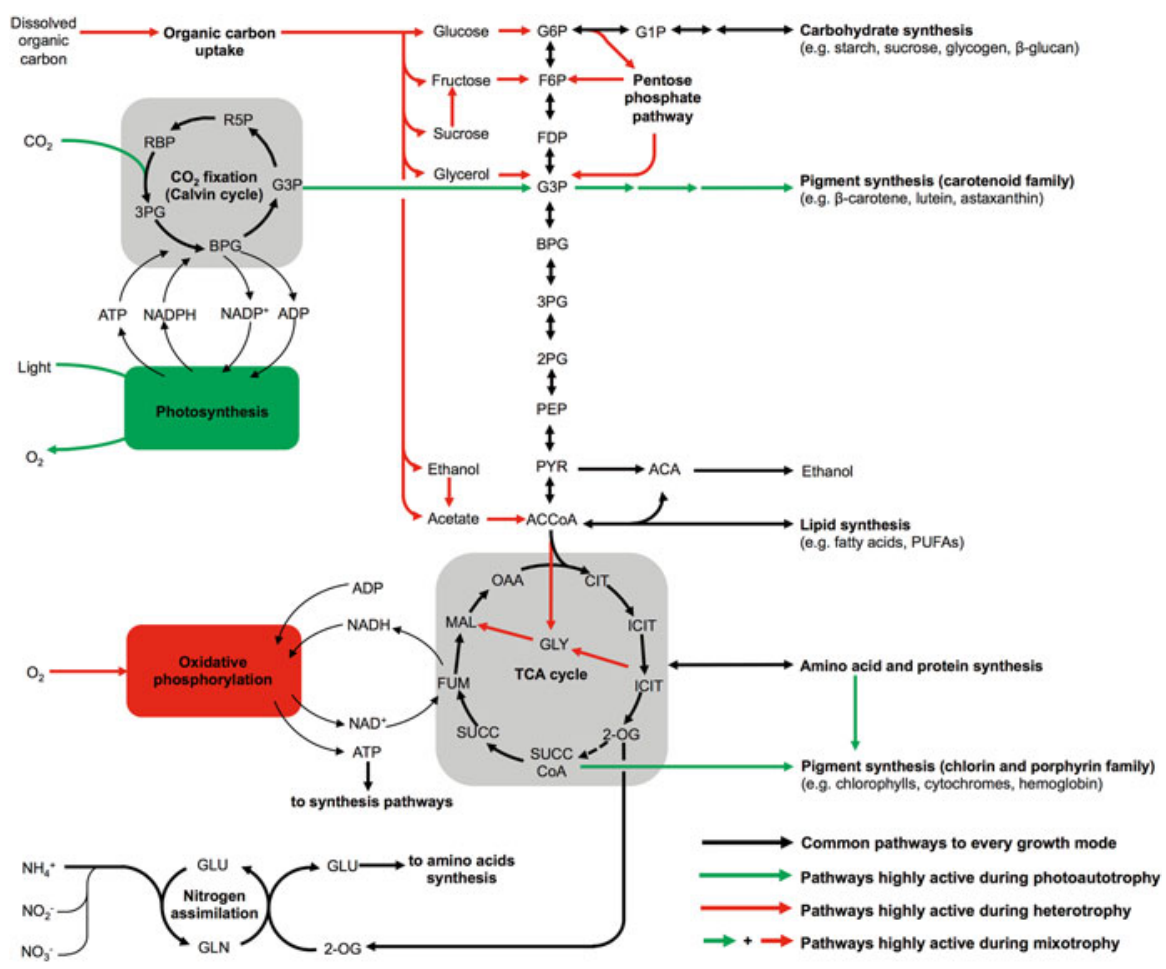

Fig. 2 Scheme of metabolic pathways for assimilation of carbon and production of energy in photoautotrophic, heterotrophic and mixotrophic microalgae. Compound abbreviations are following specified. 2-OG 2-oxoglutarate, $2 P G$ 2-phospho glycerate, $3 P G$ 3-phospho glycerate, $A C A$ acetaldehyde, $R 5 P$ ribulose-5 phosphate, $A C C O A$ acetyl-Coenzyme A, $A D P$ adenosinediphosphate, $\quad A T P$ adenosine-triphosphate, $B P G$ 1,3-biphospho glycerate, $B P G$ 1,3-bisphosphoglycerate, $C I T$ citrate, $F 6 P$ fructose-6 phosphate, $F D P$ Fructose 1,6-biphosphate, FUM fumarate, G1P glucose-1 phosphate, $G 3 P$ glyceraldehyde- 3 phosphate, $G 6 P$ glucose- 6 phosphate, $G L N$ glutamine, $G L U$ glutamate, ICIT isocitrate, $M A L$ malate, $N A D+$ nicotinamide adenine dinucleotide (oxidized), $N A D H$ nicotinamide adenine dinucleotide (reduced), $N A D P+$ nicotinamide adenine dinucleotide phosphate (oxidized), $N A D P H$ nicotinamide adenine dinucleotide phosphate (reduced), OAA oxaloacetate, $O X A$ oxalosuccinate, $P E P$ phosphoenolpyruvate, $P Y R$ pyruvate, $R B P$ ribulose-1,5 biphosphate, $S U C C$ succinate, $S U C C C O A$ succinyl-Coenzyme A

and glycogenesis (reductive EM pathway) was higher in photo-autotrophic and mixotrophic cultivation (Yang et al. 2002). The protein expression pattern under autotrophic conditions was very similar to that under mixotrophic conditions, suggesting that regulation of metabolism in these two modes occur with differences in metabolite concentrations and enzyme affinity to substrate and not at the genetic or transcriptional level (Yang et al. 2002).

Dark anaerobic cultivation of Chlamydomonas reinhardtii, Chlorella vulgaris, Chlorococcum littorale, Oscillatoria spp., Spirulina spp., and Microcystis spp. have 
been used for fermentative ethanol production (Hirano et al. 1997; John et al. 2011; Ueda et al. 1996; Ueno et al. 1998). Yet, algae cannot metabolize glucose under dark anaerobic conditions because insufficient energy is liberated during dissimilation of glucose, and low levels of the enzyme lactate dehydrogenase (EC 1.1.1.27), which is essential to complete the anaerobic fermentation process (Droop 1974; Neilson and Lewin 1974). Although these studies did not report growth under dark anaerobic conditions, they reported anaerobic endogenous respiration of storage compounds, such as starch and glycogen. Under dark anaerobic conditions, the oxidative reaction of starch is incomplete and, depending on microalgae species, $\mathrm{H}_{2}$ gas, $\mathrm{CO}_{2}$, ethanol, lactic acid, formic acid, and acetic acid are produced (John et al. 2011). Ueda et al. (1996) used microalgae biomass of the classes Prasinophyceae, Cryptophyceae, and Cyanophyceae as raw material for producing ethanol. The algal cells, containing a large amount of polysaccharides, were catabolized rapidly under dark anaerobic conditions to ethanol. Hirano et al. (1997) report that the intracellular conversion rate of starch to ethanol ranges from 0.2 to $0.15 \mathrm{~g}$ ethanol per gram of starch in Chlamydomonas reinhardtii under dark anaerobic conditions.

\subsubsection{Carboxylic Acids (Mainly Acetate)}

Uptake of dissolved carboxylic acids, such as acetic, citric, fumaric, glycolic, lactic, malic, pyruvic, and succinic, under microalgal heterotrophic cultivation, is well known for decades (Bollman and Robinson 1977). Acetate (or acetic acid) is one of the most common carbon sources for many microbial species, including microalgae (Droop 1974). The starting point for assimilating acetate is acetylation of coenzyme A by acetyl-CoA synthetase (EC 6.2.1.1). This forms acetyl coenzyme A (acetyl-CoA) in a single-step catalyzing reaction, using a single ATP molecule, (Fig. 2; Boyle and Morgan 2009; De Swaaf et al. 2003a; Droop 1974). Acetate (carried by acetyl-CoA) is generally oxidized metabolically through two pathways: the glyoxylate cycle to form malate in glyoxysomes (specialized plastids in the glyoxylate cycle) and the TCA cycle to citrate in the mitochondria, which provides carbon skeletons, energy as ATP, and energy for reduction as $\mathrm{NADH}$. By definition, microalgae that grow by assimilating acetate must possess a glyoxylate cycle pathway to efficiently incorporate acetyl groups of acetyl-CoA into carbon skeletons. The operation of the glyoxylate cycle requires synthesis of isocitrate lyase (EC 4.1.3.1) and malate synthetase (EC 2.3.3.9). Both enzymes are induced when cells are transferred to media containing acetate (Boyle and Morgan 2009; Neilson and Lewin 1974). However, acetate does not always promote growth. It is toxic for many microorganisms at high concentrations. Keeping the concentration of acetate at low levels is useful for the fed-batch configuration in cultures or $\mathrm{pH}$-auxostat, where $\mathrm{pH}$ is maintained constant (De Swaaf et al. 2003a; Zhang et al. 1999). 


\subsubsection{Alcohols (Mainly Glycerol)}

Heterotrophic growth using glycerol as a substrate has been demonstrated for microalgae species, such as Chlorella vulgaris, Nannochloropsis spp., and Schizochytrium limacinum (Table 4; Chi et al. 2007; Das et al. 2011; Liang et al. 2009). Glycerol is a source of carbon and energy for microalgae growth; it is a very compatible solute for enzymes and membranes, with almost no toxic effects even at high concentrations (Richmond 1986). Large quantities of glycerol are obtained as a by-product of the biodiesel industry; thereby it is a cheap carbon substrate for heterotrophic cultivation of microalgae (Chi et al. 2007). In microalgae cells, glycerol is first phosphorylated using ATP and the glycerophosphate is then oxidized to triose phosphate. Microalgae genomes contain genes encoding for glycerol kinase (EC 2.7.1.30), sn-glycerol-3-phosphate NAD oxidoreductase (EC 1.1.1.8), and triose-phosphate (EC 5.3.1.1) (KEGG database; see Kanehisa and Goto 2000). These enzymes convert glycerol into glyceraldehyde-3-phosphate and glycerate, which are intermediates in the EM pathway of glycolysis to form pyruvate that enters the TCA cycle. In plant cells, sn-glycerol 3-phosphate, an intermediate formed during glycerol assimilation, inhibits the glycolytic activity of the EM pathway, thereby favoring the accumulation of carbohydrates from gluconeogenesis activity (Neilson and Lewin 1974). Aubert et al. (1994) suggest that the PP pathway is also inhibited when glycerol is the only carbon source (Fig. 2). Depending on the species, microalgae heterotrophic cultivation with glycerol can achieve as high a biomass concentration, growth rate, and PUFAs production as cultures using glucose as the substrate (Chi et al. 2007). Glycerol concentration in growth medium affects the biomass composition of the cultivated microalgae. For example, carbohydrate and lipid content in C. vulgaris cells was significantly higher in heterotrophic cultures on $2 \% \mathrm{v} / \mathrm{v}$ glycerol than in cultures with $1 \% \mathrm{v} / \mathrm{v}$ (Liang et al. 2009). Glycerol can also be used to cultivate microalgae mixotrophically. Mixotrophic cultures of Nannochloropsis spp. with glycerol reached higher biomass density and lipid production than phototrophic cultures (Das et al. 2011).

\subsection{Respiration Process}

In a broad sense, all organisms, including microalgae, use the same metabolic pathways for respiration. Cellular respiration is the metabolic process to produce ATP by oxidative phosphorylation. This process involves the consumption of electron donors (reduced organic or inorganic compounds) and electron acceptors (oxidized inorganic compounds). During heterotrophic and mixotrophic growth, microalgae perform aerobic dark respiration where organic compounds are used as electron donor while oxygen molecules are consumed as final electron acceptors. In microalgae, respiration plays two major roles: It serves as the exclusive source of energy for maintenance and biosynthesis under dark conditions, and it provides essential carbon skeletons for biosynthesis under any growth condition. In microalgae, dark 
Table 3 Biomass composition ranges obtained in photo-autotrophic, heterotrophic, and mixotrophic cultivation of microalgae

\begin{tabular}{l|l|l|l}
\hline Compound (\%) & Photo-autotrophic & Heterotrophic & Mixotrophic \\
\hline \multirow{2}{*}{ Protein } & $29-60$ & $10-40$ & $32-40$ \\
\cline { 2 - 4 } & $(52.64 \pm 0.26)$ & $(10.28 \pm 0.10)$ & $(30 \pm 4.5)$ \\
\hline Carbohydrates & $10-20$ & $15-45$ & $10-30$ \\
\cline { 2 - 4 } & $(10.62 \pm 0.14)$ & $(15.43 \pm 0.17)$ & $(20 \pm 5.0)$ \\
\hline \multirow{2}{*}{ Lipids (total) } & $3-28$ & $25-60$ & $11-58$ \\
\hline Ash & $(14.57 \pm 0.16)$ & $(55.20 \pm 0.20)$ & $(33 \pm 3)$. \\
\hline Others (nucleic acids and pigments) & $(6.36 \pm 0.05)$ & $(5.93 \pm 0.04)$ & $(7.00 \pm 0.26)$ \\
\hline
\end{tabular}

Numbers in parenthesis are averages reported for Chlorella protothecoides growing on glucose (Xu et al. 2006; Heredia-Arroyo et al. 2010)

respiration of an organic substrate assimilated from the medium has rates varying from 0.01 to 0.6 day $^{-1}$ (Perez-Garcia et al. 2011b). Respiration rates associated with assimilation of organic substrates vary through the cell cycle and complement $\mathrm{O}_{2}$ consumption rates associated with endogenous respiration, that is, oxidation of an intracellular storage compound, such as starch (Lloyd 1974).

The respiration rate of any organic substrate is intimately geared to growth and cell division. Therefore, dark respiration rates increase with growth rates. Under optimal conditions, respiration rates are about $20-30 \%$ of growth rates (Geider and Osborne 1989). Physiological regulation of respiration is assumed to be controlled by demand for the products of respiration metabolism, such as energy in the form of ATP and NADH and carbon skeletons provided by the organic substrate (Geider and Osborne 1989). With heterotrophic growth conditions, respiration rates equal or exceed the theoretical minimum cost of biomass synthesis. Values for $\mathrm{CO}_{2}$ generated per carbon incorporated into new biomass $\left(\mathrm{CO}_{2} / \mathrm{C}\right)$ ranged from 0.4 to 1.4 for several Chlorella species and diatoms. This indicates that biomass synthesis during heterotrophic growth conditions can proceed at nearly maximum theoretical efficiency. This happens since $\mathrm{CO}_{2} / \mathrm{C}$ ratios for autotrophic growth are much lower than in heterotrophic growth (Raven 1976). Cellular assimilation of organic compounds is influenced by the amount of $\mathrm{CO}_{2}$ dissolved in the culture's growth medium; a decline in organic matter per cell weight produced was greater when the supply of $\mathrm{CO}_{2}$ was low (Pipes and Gotaas 1960).

\subsection{Biomass Composition}

The growth regime (photo-autotrophic, heterotrophic, or mixotrophic), together with the microalga strain and supplied organic substrate, greatly influences the molecular composition of microalgae biomass. The composition of biomass is usually reported as the percentage of biomass per dry weight of protein, lipids, or carbohydrates or any other specific molecule. Table 3 presents percentage ranges of dry 
weight biomass for the main cellular components reported under photo-autotrophic, heterotrophic, or mixotrophic cultivation of various species (Cheirsilp and Torpee 2012; Cheng et al. 2009a; Lu et al. 2010; Park et al. 2012; Xu et al. 2006). In general, energy storage molecules, such as lipids and carbohydrates (starch and glycogen) are accumulated under heterotrophic and mixotrophic conditions; therefore, the content in biomass of these compounds is higher than under photo-autotrophic conditions (Choix et al. 2012a, 2014). For example, lipid content in Chlorella protothecoides cultured under heterotrophic conditions with different carbon sources always reached from 50 to $60 \%$ of cells dry weight in comparison to $15 \%$ reached under photo-autotrophic conditions (Table 3) (Cheng et al. 2009a; Xiong et al. 2008; Lu et al. 2010; Xu et al. 2006; Yan et al. 2011; Leyva et al. 2014, 2015).

Mixotrophic cultivation of various microalgae species promotes a higher percentage of lipids in biomass in comparison to photo-autotrophic cultivation. Several examples show this phenomenon in various species of microalgae. Park et al. (2012) isolated 14 Chlorophyta strains from a cold northern Canadian province and compared their biomass composition. All newly isolated strains produced higher lipid content under mixotrophic cultivation than under photo-autotrophic cultivation. Similarly, lipid production in mixotrophic cultures of Chlorella sp. and Nannochloropsis sp. was notably higher than under photo-autotrophic and heterotrophic cultivation (Cheirsilp and Torpee 2012). C. sorokiniana was found to be well suited for lipid production. This was based on its high biomass production rate and lipid content, reaching $51 \%$ under mixotrophic conditions. In this study, expression levels of $a c c \mathrm{D}$ (heteromeric acetyl-CoA carboxylase beta subunit), acc1 (homomeric acetyl-CoA carboxylase), and $r b c \mathrm{~L}$ (ribulose 1, 5-bisphosphate carboxylase/oxygenase large subunit) genes were studied with real-time PCR. Increased expression levels of $a c c \mathrm{D}$ reflect the increased lipid content in the stationary phase of mixotrophic growth, but expression of the acc1 gene remained low. This result suggests that this gene may not be critical for accumulating lipids. Additionally, reduction of expression of the $r b c \mathrm{~L}$ gene during mixotrophic conditions on glucose indicated that using glucose reduces the importance of this gene during photosynthesis (Wan et al. 2011). Compared with photo-autotrophic cultivation, lipid productivity of Nannochloropsis sp. under mixotrophic cultivation with glycerol was improved by 40 to $100 \%$ (Das et al. 2011). Supplemented with glucose, lipid productivity of Nannochloropsis oculata, Dunalliela salina, and Chlorella sorokiniana under mixotrophic cultivation were 1.1-1.6 times, 1.8-2.4 times, and 4.1-8.0 times more productive than under photo-autotrophic cultivation, respectively (Chojnacka and Noworyta 2004). Production of lipids by Scenedesmus obliquus, under mixotrophic cultivation with a supply of glucose, could be as high as $270 \mathrm{mg} \mathrm{L}^{-1} \mathrm{day}^{-1}$, which is about 50 times greater than production under photo-autotrophic cultivation (Wang et al. 2014). Cheng et al. (2009a) compared lipid production of Chlorella vulgaris under photo-autotrophic, mixotrophic, and heterotrophic cultivation. Experimental results found that, by adding $1 \%(\mathrm{w} / \mathrm{v})$ glucose, production of lipids by $C$. vulgaris under mixotrophic cultivation was, respectively, 1.5 times and 13.5 times greater than under heterotrophic and photo-autotrophic cultivation.

Even though biomass production and lipid contents in biomass are affected by the microalgae's growth regime, the composition of lipids is not greatly affected. 
Fatty acid (reported as fatty acids methyl esters, FAMEs) composition profiles in Chlorella spp. in studies of photo-autotrophic, heterotrophic, and mixotrophic cultivation is dominated by three fatty acid methyl esters: palmitic acid methyl ester $\left(\mathrm{C}_{17} \mathrm{H}_{34} \mathrm{O}_{2}\right)$, linoleic acid methyl ester $\left(\mathrm{C}_{19} \mathrm{H}_{34} \mathrm{O}_{2}\right)$, and oleic acid methyl ester $\left(\mathrm{C}_{19} \mathrm{H}_{36} \mathrm{O}_{2}\right)$. These fatty acids make up $70-90 \%$ of the FAMEs under all tested conditions (Cheirsilp and Torpee 2012; de-Bashan et al. 2002a; Gao et al. 2010; Leyva et al. 2015; Lu et al. 2010; Rosenberg et al. 2014; Xu et al. 2006; Zhang et al. 2014). Not all fatty acids in the lipids can be transferred to FAMEs (biodiesel), since the percentage of lipids suitable for biodiesel production, namely saponifiable lipids, varies with strains and cultivating conditions. Thus, the productivity of saponifiable lipids should be regarded as a more precise measurement of microalgal biodiesel productivity potential (Wang et al. 2014).

Nitrogen depletion generally leads to lipid and carbohydrates accumulation (Brányiková et al. 2011; Choix et al. 2012a, b, 2014; Dragone et al. 2011; Illman et al. 2000; Khozin-Goldberg and Cohen 2011; Leyva et al. 2015; Li et al. 2008). For example, the lack of a nitrogen source (urea) increases the lipid content in Chlorella spp. (Hsieh and Wu 2009). Lack of a nitrogen source is the main reason why $C$. sorokiniana cells shifted from growth phase to stationary phase with high lipid content in mixotrophic cultures (Wan et al. 2011). When nitrogen is not present, accumulation of lipids is attributed to mobilization of lipids from chloroplast membranes by the enzyme 1,5-biphosphate carboxylase/oxygenase (EC 4.1.1.39, Rubisco) (García-Ferris et al. 1996). This is supported by the fact that development of chloroplasts and their light harvesting chlorophyll-protein complexes is dependent on nitrogen. Breakdown of chloroplasts for internal supply of nitrogen under nutrient reduction and dark conditions leads to cell survival and growth in the face of prolonged nutrient shortage if an external carbon source is not supplied. Batch cultivation experiments with $C$. vulgaris showed that acetyl-CoA carboxylase (EC 6.4.1.2) activity is directly related to fatty acid accumulation, especially in the final days of cultivation, when nitrogen levels in the medium are depleted (Leyva et al. 2014, 2015). Limiting $\mathrm{N}$ or $\mathrm{P}$ also influences the accumulation of carbohydrates in microalgae cells (Dragone et al. 2011). Brányiková et al. (2011) states that to produce biomass with high starch content, it is necessary to suppress cell division by limiting $\mathrm{N}$ or $\mathrm{P}$, but not disturbing synthesis of starch in chloroplasts. These examples refer to work with Chlorella spp. Nutrient starvation has similar effects on lipid and carbohydrate accumulation in other microalgae, such as Monodus subterraneus, Nannochloropsis sp., and Parietochloris incisa (Khozin-Goldberg and Cohen 2006; Merzlyak et al. 2007; Pal et al. 2011; Solovchenko et al. 2008). Carbohydrate composition in Chlorella, specifically starch content, is modified when it is immobilized with the plant growth-promoting bacteria Azospirillum brasilense (Choix et al. 2012a, b, 2014). Heterotrophic cultivation with glucose or acetate of this coimmobilized cell system had higher total carbohydrate and starch content per culture and per cell than cultures with Chlorella spp. immobilized alone. The presence of $A$. brasilense cells in the immobilized system significantly prolonged production of starch in comparison to cultures with Chlorella spp. immobilized alone. These results demonstrate that microalgae immobilized with specific beneficial bacteria is a promising strategy to enhance productivity of desirable compounds in microalgae cultures. 


\subsection{Response to Light in Mixotrophic Metabolism}

During mixotrophic cultivation, microalgae can grow both photo-autotrophically and heterotrophically and can use inorganic and organic carbon sources. Inorganic carbon is fixed through photosynthesis, which is influenced by illumination conditions, while organic compounds are assimilated through aerobic respiration, which is affected by the availability of organic carbon and oxygen (Wang et al. 2014). Since organic compounds can be assimilated under mixotrophic cultivation, growth of microalgae does not strictly depend on photosynthesis; hence light is not an absolute limiting factor for growth. Growth limitation by low illumination and growth photo-inhibition by high intensity of light can be significantly reduced in mixotrophic cultures (Wang et al. 2014). Photo-oxidative damages (especially in closed photobioreactors, where oxygen accumulation occurs) may also be reduced because of oxygen consumption during the heterotrophic metabolism. This compensates for increased oxygen production of the photo-autotrophic metabolism; thereby, oxygen cannot reach toxic levels (Chojnacka and Marquez-Rocha 2004; Vonshak et al. 2000).

Mixotrophic cultures have a $20-40 \%$ higher growth rate at any given light intensity in comparison with photo-autotrophic cultures (Vonshak et al. 2000). Cells of the cyanobacteria Spirulina platensis grown under mixotrophic conditions exhibit a modified metabolic response to light in comparison to the one exhibited by cells growing under photo-autotrophic conditions (Vonshak et al. 2000). For example, its maximum photosynthetic rate and light saturation value in mixotrophic cultures were higher than those of the photo-autotrophic cultures. Dark respiration and light compensation point were also significantly higher in these cells. As expected, the mixotrophic cultures grew faster and achieved a higher concentration of biomass than the photo-autotrophic cultures. In contrast, the growth rate of the photoautotrophic cultures was more sensitive to light. The differences between the two cultures were also apparent in their responses to exposure to very high photon flux density of $3000 \mu \mathrm{mol} \mathrm{m} \mathrm{m}^{-2} \mathrm{~s}^{-1}$. The light-dependent $\mathrm{O}_{2}$ production rate and the maximal efficiency of photosystem II declined more rapidly in photo-autotrophically grown cells than in mixotrophically grown cells, as a result of exposure to high photon flux density. Although both cultures recovered from the high photon flux density stress, the mixotrophic culture recovered faster and reached a higher concentration of biomass. Based on these results, growth of $S$. platensis with a fixed carbon source has a significant effect on photosynthetic activity (Vonshak et al. 2000).

Another interesting aspect is the reduction, or even complete stopping, of photoinhibitory effects while microalgae are growing mixotrophically. This is explained either by the protective influence of the organic nutrient on cell metabolism or the increase of light intensity required to photo-inhibit cell growth (Chojnacka and Noworyta 2004). Presence of light, however, can also photo-inhibit uptake of organic carbon by affecting the balance between reduced and oxidized energycarrying molecules (ATP and NADH), as a consequence of photosynthetic activity. For example, in C. kessleri cultures, high light intensities (above $120 \mu \mathrm{mol} \mathrm{m}^{-2} \mathrm{~s}^{-1}$ ) inhibited consumption of organic soluble compounds in a growth medium based on wastewater. However, consumption of soluble compounds was independent of light intensity in C. protothecoides (Li et al. 2012). If large-scale cultivation is the ulti- 
mate goal, only those microalgae strains which are not sensitive to photo-inhibition of organic carbon uptake are suitable for mixotrophic cultivation (Li et al. 2012).

The effect of light intensity on accumulating biomass, removing wastewater nutrients and producing biodiesel through microalgae mixotrophic cultivation, was investigated using $C$. kessleri and $C$. protothecoides (Li et al. 2012). The experimental light intensities were $0,15,30,60,120$, and $200 \mu \mathrm{mol} \mathrm{m} \mathrm{m}^{-2} \mathrm{~s}^{-1}$. The results show that light intensity had a profound impact on responses in both species. The dependence of these responses on light intensity varied. For C. kessleri, optimum light intensity was $120 \mu \mathrm{mol} \mathrm{m}{ }^{-2} \mathrm{~s}^{-1}$ for biomass productivity and removal of $\mathrm{NH}_{3}-\mathrm{N}$ and $\mathrm{PO}_{4}$-P, except for removal of organic carbon measured as chemical oxygen demand (COD). For C. protothecoides, optimum light intensity was $30 \mu \mathrm{mol} \mathrm{m} \mathrm{m}^{-2} \mathrm{~s}^{-1}$. Light intensity also greatly affects the composition and quantity of biodiesel derived from algae cultivated under different lighting conditions. The major components of biodiesel produced from microalgae biomass were 16-C and 18-C fatty acids methyl esters (FAME). The highest biodiesel contents were $24.19 \%$ and $19.48 \%$ of dried biomass for $C$. kessleri and C. protothecoide, respectively. Both species were capable of removing wastewater nutrients with high removal efficiencies under all lighting conditions ( $\mathrm{Li}$ et al. 2012).

Mixotrophic cultivation is also associated with lower emission of $\mathrm{CO}_{2}$ than heterotrophic cultivation on the basis of per unit biomass/lipid production. This happens because part of the $\mathrm{CO}_{2}$ release can be compensated by photosynthesis (Chen et al. 2011; Xiong et al. 2010). Compared with heterotrophic cultivation, mixotrophic cultivation of $C$. protothecoides released $61.5 \%$ less $\mathrm{CO}_{2}$ with production of the same yield of lipid (Xiong et al. 2010). Nevertheless, the enzyme Rubisco, which is responsible for $\mathrm{CO}_{2}$ fixation, remains functional in cells at the heterotrophic phase (Xiong et al. 2010). During mixotrophy, growth is influenced by the medium supplement of glucose during both the light and dark phases; hence, there is less loss of biomass during the dark phase characterized by $\mathrm{CO}_{2}$ emission (Wang et al. 2014).

\subsection{Advances of Metabolic and Physiological Characteristics of a Strain}

One aspect often mentioned to overcome bottlenecks in microalgal cultivation is to produce biomass using a high-performance strain. Ideally, microalgae strains used for bio-refining of valuable compounds should present the following metabolic and physiological characteristic (Brennan and Owende 2010; Wijffels et al. 2010):

- High production of biomass (fast growth)

- High production of a valuable compound (high percentage of compound weight per unit of biomass)

- Able to growth heterotrophically, using cheap carbon substrates

- High biomass or valuable compound yield per unit of light and/or organic substrate 
- Robust growth (able to maintain productivity under changing conditions)

- Good ecological dominance (able to maintain high productivity under non axenic conditions)

- Growth at a wide range of $\mathrm{pH}$

- High $\mathrm{CO}_{2}$ sinking capacity

- Insensitivity to oxygen (resist photo-oxidative stress)

- Self-flocculation characteristics

- Excretion from the cells of valuable compounds (to facilitate product extraction)

- Large cells with thin cell walls (to facilitate product extraction)

- Resistant to possible inhibitory effect of organic compounds

Currently, none of the known microalgal strains is capable of meeting all these requirements with high efficiency. Optimization of these characteristics can lead to a robust and efficient process of scaling-up and eventually to successful commercialization of products. In general, there are two possible approaches to improve the metabolic and physiological characteristics of biorefinery microalgae cultures: isolation of strains with desirable characteristics (bio-prospecting) and, designing improved strains by genetic/metabolic engineering.

\subsection{Bio-prospecting of Species for Valuable Compound Production}

Each microalgal species and strain has different capacities to assimilate different organic compounds, and these characteristics must be considered when determining its use in producing desirable compounds (Kröger and Müller-Langer 2011). Only a few microalgal strains are cultivated commercially for various high-value products. These include: Chlorella prothotecoides, Crypthecodinium cohnii, Dunaliella salina, Haematococcus pluvialis, and Nannochloropsis gaditana. These are not necessarily the best strains for the production of cheap chemicals, such as biodiesel. Consequently, it is necessary to search for new strains or modify the strains so that production of valuable compounds becomes economically viable. In this context, efforts at bio-prospecting of heterotrophic microalgae should focus on: testing strains under several carbon sources at different concentrations (like those tests depicted on Fig. 3), including testing under mixotrophic conditions and testing for growth on cheap organic substrates, such as food waste, industrial by-products, or industrial waste.

Barclay and Apt (2013) and Charles et al. (2002) described approaches and protocols for bio-prospecting and isolating microalgae from natural habitats. These include bio-prospecting strategies focused on: (a) broad collections of algae; (b) collections on specific habitat types; (c) isolating extremophilic microalgae; (d) a target chemical composition or product; (e) heterotrophic growth potential; (f) wastewater tolerance; $(\mathrm{g})$ pesticide tolerance, and $(\mathrm{h})$ targeting various strain capa- 

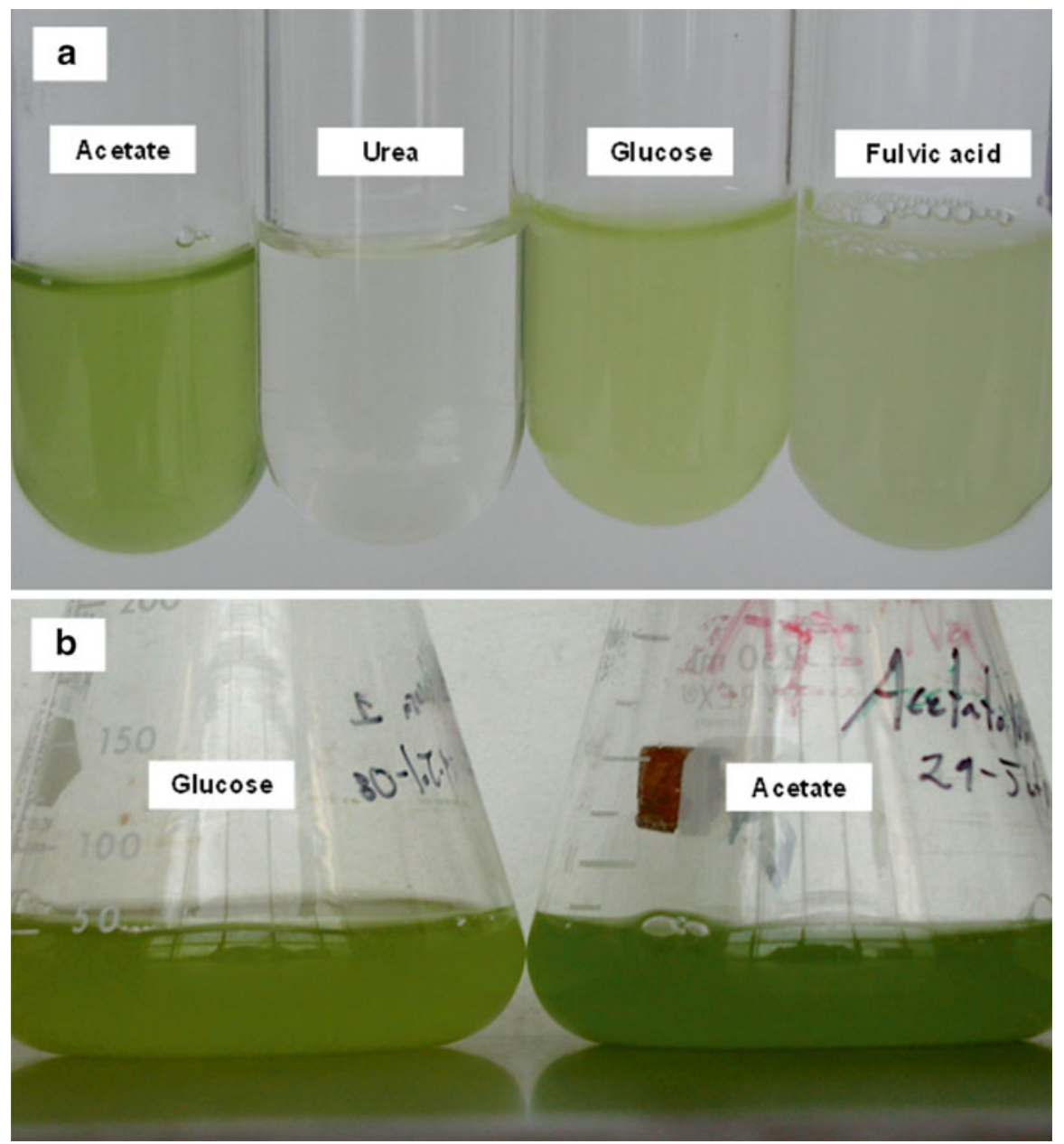

Fig. 3 Bio-prospecting heterotrophic capabilities of Chlorella vulgaris UTEX 2714 under completely dark conditions: (a) $50 \mathrm{~mL}$ tube-cultures after 4 days of cultivation on different organic substrates, initial concentration of substrate was constant in all cultures and (b) $250 \mathrm{~mL}$ flask cultures

bilities. Serial plating (Park et al. 2012) and serial dilution (Bhatnagar et al. 2011) are two common isolation methods; however, more innovative approaches have been successfully implemented. For example, selection of "xylose-enhanced" strains was carried out by cyclic exposure to UV radiation and fluorescent light of microplates containing Chlorella spp. (Hawkins 1999).

Bio-prospection can lead to the discovery of microalgae with novel heterotrophic growth capabilities. Girard et al. (2014) tested the capability of Scenedesmus acutus, Scenedesmus obliquus, Chlorella vulgaris, and Chlorella protothecoides to grow on lactose and cheese whey as carbon sources under heterotrophic and mixotrophic conditions. They found that only Scenedesmus obliquus can use 
these substrates for growth. Wang et al. (2010) isolated a Chlorella sp. strain tolerant of high nitrogen loads (81-178 $\mathrm{NH}_{4}-\mathrm{N} \mathrm{mg} \mathrm{L}^{-1}$ ) in dairy manure. Chlorella sp. was able to remove all the nitrogen under heterotrophic cultivation within 21 days. Three mixotrophic microalgae strains of Chlamydomonas globosa, Chlorella minutissima, and Scenedesmus bijuga were isolated after long-term enrichment of cultures containing industrial wastewater (Bhatnagar et al. 2011). In another example, native microalgal strains were isolated from carpet industry wastewater. Some of the isolated strains had heterotrophic growth capability and also reduced the nutrient content on medium (Chinnasamy et al. 2010). Park et al. (2012) analyzed biomass and lipid production of 14 microalgae isolates (in the Chlorophyta family). Each isolated strain has a distinctive linear correlation between biomass and lipid productivity under photoautotrophic and mixotrophic conditions, but only one strain (Chlamydomonas debaryana-AMLs1B) held promise for biorefinery purposes. So far, bio-prospecting for strains for heterotrophic and mixotrophic growth has potential, but few strains were isolated to show the usefulness of this approach.

\subsection{Genetic and Metabolic Engineering for Improving Existing Strains}

Genetic and metabolic engineering can be used to expand heterotrophic and mixotrophic capabilities of strains by enabling a strain to growth on a specific organic substrate or developing a metabolic pathway for a novel product synthesis by inserting the genes for these pathways. Genetic and metabolic engineering are likely to improve the metabolic performance of microalgal strains producing valuable compounds during the coming decades (Brennan and Owende 2010; León-Bañares et al. 2004; Wang et al. 2009). There is an increasing interest in use transgenic microalgae as green cell factories capable of producing biofuels and valuable proteins and carbohydrates (León-Bañares et al. 2004). Nowadays few microalgae, including the green algae Chlamydomonas reinhardtii, Nannochloropsis gaditana, and Ostreococcus tauri or the diatom Phaeodactylum tricornutum are currently established as platforms for genetic and metabolic engineering (Wijffels et al. 2013). Cellular transformation techniques have been much better developed in cyanobacteria and transformation systems need to be further developed for microalgae (Wijffels et al. 2013). A promising strategy is transformation of heterologous (prokaryotic and eukaryotic) genes in established eukaryotic hosts, such as Chlamydomonas reinhardtii (Wijffels et al. 2013).

Successful metabolic engineering of strains involves the sequencing of a wild type strain, a systematic description of the organism genotype and phenotype using 'omics' techniques, then the application of molecular and genetic techniques for metabolic engineering. Metabolic engineering of a specific strain has one of the following goals: (a) expand metabolic capabilities by adding new genes to an organism; (b) add genes encoding for transporters of products and intracellular toxic compounds so cells can excrete them faster; (c) increase formation of a pre- 
cursor of a valuable compound; (d) remove precursor catabolism; (e) promote synthesis and storage of compounds in intracellular compartments; and (f) promote efficient product-secretion signaling to avoid product feedback inhibition (Lee et al. 2008; Wang et al. 2009; Wijffels et al. 2013). Metabolic engineering tools to achieve these goals include: insertion of heterologous genes in the genome; insertion of heterologous genes extra-chromosomally via conjugation with replicable plasmids; resistance markers; and random mutagenesis procedures (León-Bañares et al. 2004). Using these methods, various de novo metabolic pathways have been introduced into cyanobacteria species, such as Synechocystis spp. and Synechococcus spp. (Ducat et al. 2012; Gao et al. 2012) and the microalgae Nannochloropsis gaditana (Radakovits et al. 2012). To gain the best possible insight into metabolic pathways leading to the product of interest, systems biology, a bioengineering research field based on computational and mathematical modeling of complex biological systems, is used to integrate data from culture's performance measurements and diverse state-of-the-art technologies. These include genome sequencing, transcriptomics, metabolomics, proteomics, metabolic modeling (fluxomics), and bioinformatics.

Two key aspects for successful application of metabolic engineering are the availability of well-annotated genomes and the availability of quantitative tools for metabolic modeling that permit understanding and manipulation of the genome. Metabolic models, but most specifically stoichiometric metabolic network (SMN) models, are at the core of the systems biology approach by providing a computational platform for omics data analysis and phenotype prediction. Figure 4 presents the metabolic network formed by a model of Chlamydomonas reinhardtii metabolism developed by Chang et al. (2011). This network accounts for 1080 genes associated with 2190 reactions and 1068 unique metabolites. SMN models have mainly focused on the derivation of flux-balance analysis or FBA (Orth et al. 2010), which can be used either for designing the bioreactor's operation conditions or targeting metabolic reactions and genes for metabolic engineering (Oberhardt et al. 2009). For example, Perez-Garcia et al. (2014) used a metabolic network model of the ammonia-oxidizing bacteria Nitrosomonas europaea to find bioreactor operation conditions to avoid the emission of greenhouse gases from biological nitrogen-removal processes.

Legal regulations for large-scale outdoor production of genetically modified microalgae are not yet in place, but efforts to establish regulations are in progress. As a starting point, it is necessary to provide risk assessment studies before outdoor industrial applications of genetically modified cyanobacteria and microalgae (Wijffels et al. 2013). An argument against genetic engineering of strains is that many microorganisms suitable for outdoor mass culture are yet to be discovered; therefore, development of new strains is unnecessary (Rodolfi et al. 2009). Nevertheless, it may be prudent to limit projections to what can be achieved with natural strains. For example, the Aquatic Species Program of the US Department of Energy collected over 3000 strains of oil-producing photosynthetic microorganisms, which after screening, isolation, and characterization efforts, narrowed the collection of promising organisms to 300 species, mostly green algae and diatoms (Sheehan et al. 1998). 


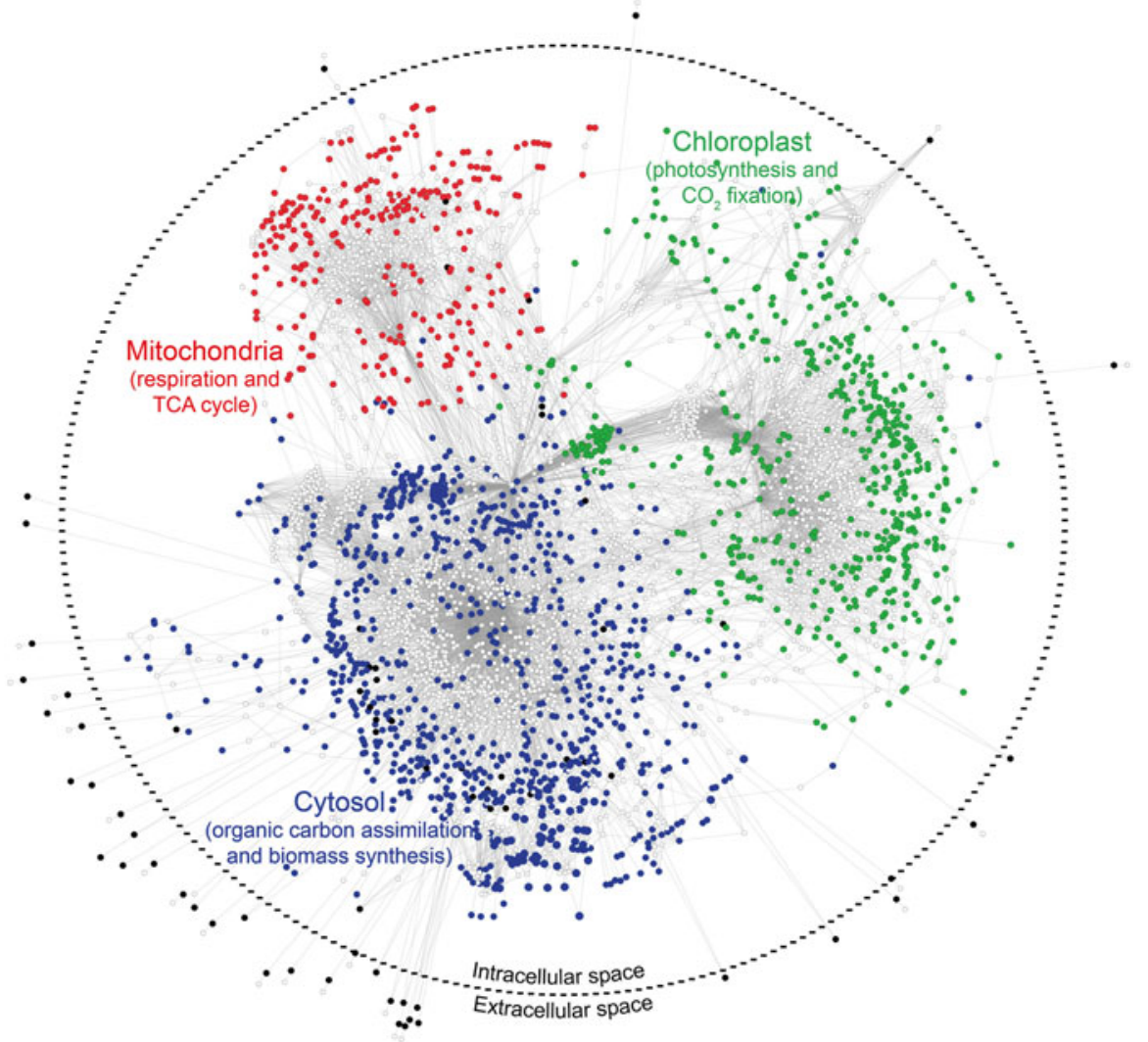

Fig. 4 Genome scale metabolic network of Clamydomonas reinhardtii used for systems biology and metabolic engineering studies. The big circle formed by a slashed line represent the boundary of cellular metabolism. Intracellular blue nodes represent metabolites occurring in cytoplasm, Golgi apparatus, nucleus and glyoxysome; green nodes represent metabolites occurring in chloroplast and thylakoid lumen; red nodes represent metabolites occulting in mitochondria; black nodes on the "extracellular space" represent metabolites able to be assimilated and/or secreted from the growth medium, white small nodes represent metabolic reactions, grey edges connect metabolic compounds with reactions; the metabolic network was created for the $C$. reinhardtii metabolic model published by Chang et al. (2011) using the software Cytoscape 3.0.1 (Cytoscape consortium, San Diego, USA)

\section{Organic Carbon Substrates}

\subsection{Bulk Organic Compounds}

Although heterotrophic and mixotrophic microalgae can use a wide range of carbon and nitrogen sources, from a commercial perspective, the most economic organic substrates for heterotrophic cultivation are glucose, glycerol, and acetate (Barclay et al. 2013; Perez-Garcia et al. 2011b). However many carbon sources, such as 
arabinose, citrate, fructose, malate, lactic acid, lactose, peptone, urea, fulvic acids, ethanol, methanol, and sucrose, have been tested for heterotrophic cultivation of microalgae. Chlorella vulgaris cultures on the above mentioned carbon sources reached significantly lower biomass concentration in comparison to cultures on acetate or glucose (Perez-Garcia et al. 2011a). Microalgal cells growing on "less preferred" substrates require a lag time (acclimation period) to develop the specific transport systems necessary for compound uptake. For example, consumption of "less preferred" substrates is aborted because the enzymes that catalyze their uptake and assimilation cannot be synthesized in the presence of the preferred substrate in Crypthecodinium cohnii (Ratledge and Wynn 2002). Lag time is also dependent on the strain, bioreactor configuration, and environmental growth conditions.

Although using glucose, acetate, or glycerol as organic substrate is pertinent for research, these compounds are too expensive for an economically viable bio-refinery production process. For example, glucose is about $80 \%$ of the costs of the medium, when cultivating heterotrophic Chlorella spp. for biodiesel production (Kröger and Müller-Langer 2011). Alternative, low-cost substitutions to glucose, such as starch and cellulose-hydrolyzed solutions are ideal carbon sources for heterotrophic microalgae cultivation because similar biomass production can be achieved as using glucose, but at a lower cost (Li et al. 2007). According to two rigorous techno-economic analyses (Liang 2013; Tabernero et al. 2012), production of biofuel and valuable chemicals using heterotrophic and mixotrophic microalgae is not viable if the carbon source is a pure chemical or is it based on land crops, such as sugarcane, established to supply microalgae media. Production of valuable compounds by heterotrophic and mixotrophic microalgae are only viable if organic substrates are supplied from wastes or by-products of other processes. Consequently, studies have investigated microalgae growth on several alternative organic substrates, such as wastewater, industrial by-products, and lignocellulosic materials. The following sections discuss the main findings of those studies.

\subsection{Wastewater}

Most domestic and industrial wastewaters contain organic carbon, nitrogen, phosphorus, and other minor compounds. This composition makes wastewater suitable for growing microalgae. Besides growing microalgal biomass for valuable compound production, the wastewater is simultaneously treated (Christenson and Sims 2011; Rawat et al. 2011). This double benefit has attracted extensive attention in recent years. Yet, this approach has its drawbacks: (a) some wastewater may be too toxic to support microalgal growth; (b) with outdoor treatment, microalgal growth and wastewater treatment efficiency is significantly affected by seasonal changes; and (c) competition among the microbial community in the wastewater may lead to very slow algal growth. Hence, using microalgae to treat wastewater is problematic for high biomass productivity (Liang 2013; Valderrama et al. 2002). 
Soluble organic compounds in wastewater can be assimilated by heterotrophic and mixotrophic microalgae. Organic carbon in wastewater is usually reported as chemical oxygen demand (COD). Heterotrophic and mixotrophic activity in wastewater is usually inferred from the decrease of COD concentration during experimental cultivation ( $\mathrm{Hu}$ et al. 2012; Li et al. 2011). Hu et al. (2012) observed a decrease in COD concentration from 2.5 to $0.5 \mathrm{~g} \mathrm{~L}^{-1}$ in mixotrophic cultures of Auxenochlorella protothecoides in municipal wastewater. This cultivation presented a COD removal efficiency of $78.9 \%$. Probably the remaining $0.5 \mathrm{~g} \mathrm{~L}^{-1}$ of COD belonged to the non-biodegradable COD fraction. Chlorella pyrenoidosa, growing in sterilized sewage, was able to use some of the organic matter, as indicated by a decrease in soluble biological oxygen demand (BOD) and dissolved volatile solids (VSS). However, not all organic chemical substances in the polluted or unpolluted environments can be used as a carbon source for microalgae since some are known to inhibit microalgal growth (Chen 1996; Perez-Garcia et al. 2011a). Various studies show that supplementing readily-biodegradable carbon sources, such as glucose or acetate, is necessary to achieve the dual goal of high nutrient removal efficiency and high biomass production (Perez-Garcia et al. 2011a; Rawat et al. 2011). Naturally, adding extra nutrients for the purpose of increasing biomass production can lead to a wastewater even more polluted than the original one, even after cultivation of the microalgae. Nitrogen removal from wastewater can be achieved by heterotrophic and mixotrophic cultivation of microalgae (Hu et al. 2012; Perez-Garcia et al. 2010, 2011a). Hu et al. (2012, 2013) observed ammonium removal efficiencies from 52 to $72 \%$ in mixotrophic cultures of Auxenochlorella protothecoides and Chlorella sp. Similarly, total nitrogen removal efficiencies ranged from 64 to $74 \%$. Removing phosphorus from wastewater can also be achieved with heterotrophic and mixotrophic cultivation of algae. For example, mixotrophic cultures of $A$. protothecoides removed $75 \%$ of the initial phosphorus concentration of $125 \mathrm{mg}-\mathrm{PO}_{4} \mathrm{~L}^{-1}$ in municipal wastewater (Hu et al. 2012). In their study, removing phosphorus from mixotrophic cultures was a consequence of microalgae uptake, together with chemical precipitation of dissolved phosphorus (orthophosphate).

Under realistic conditions of cultivation, to increase the algae competitiveness against fast-growing native bacteria in the wastewater and, at the same time, produce high quantities of biomass and lipids is still a major research challenge. The use of a microalgae consortium may increase the robustness of a microalgae population in wastewater under any cultivation regime. Photo-autotrophic mixed cultures, dominated by $C$. vulgaris populations, showed higher biomass and lipid production than photo-autotrophic pure $C$. vulgaris cultures. In addition, lipid production in photo-autotrophic mixed cultures was higher than that in heterotrophic culture (Table 4, Zhang et al. 2014). A microalgal consortium, mostly formed by Chlorella, Chlamydomonas, Scenedesmus, and Gloeocystis species cultivated in wastewater from the carpet industry, using raceway ponds, showed higher biomass production and removal of nitrogen and phosphorus than 13 pure strains (Chinnasamy et al. 2010).

Using competitive local strains from wastewater environments is another option. For example, native microalgae isolated from carpet industry wastewaters, such as 


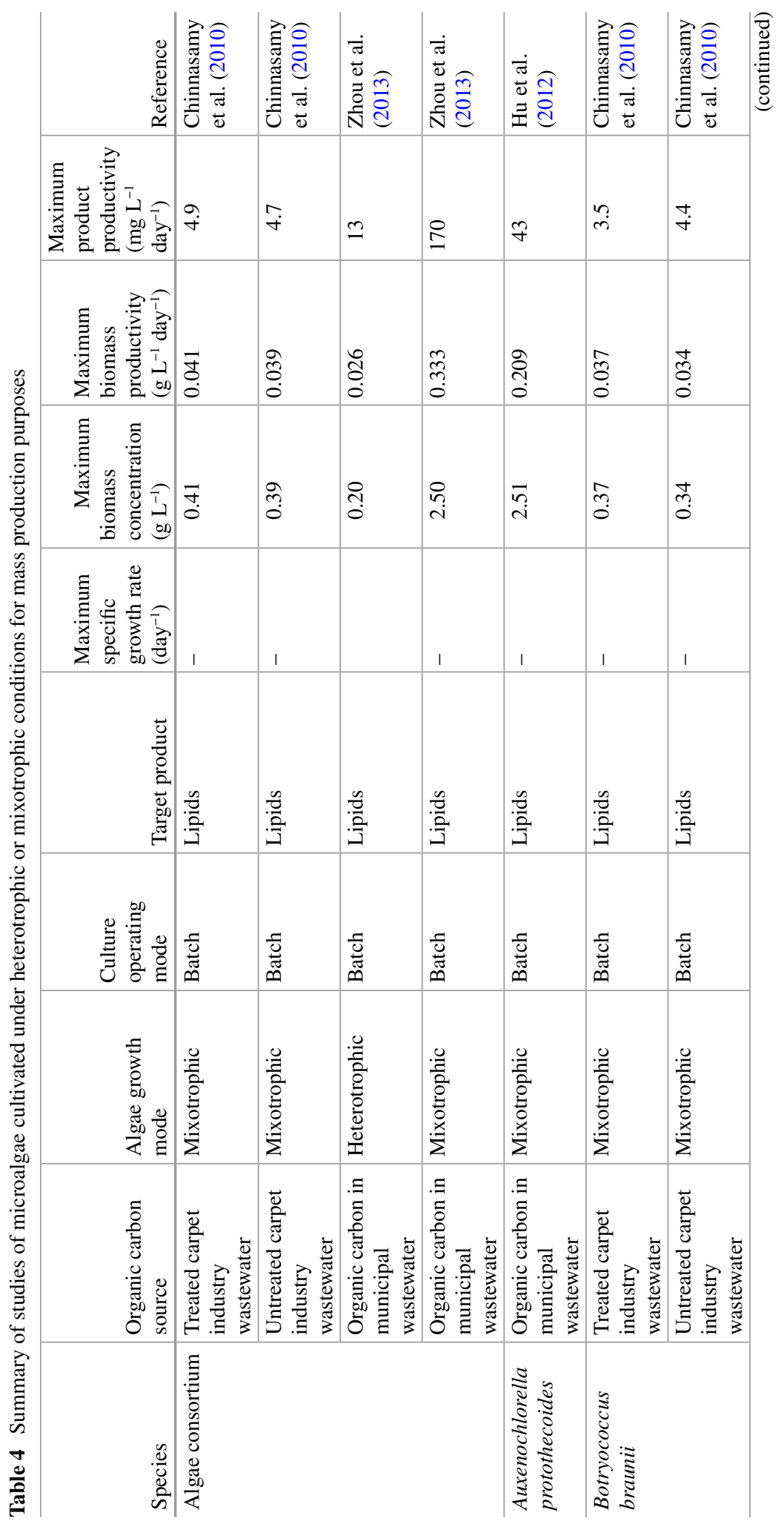




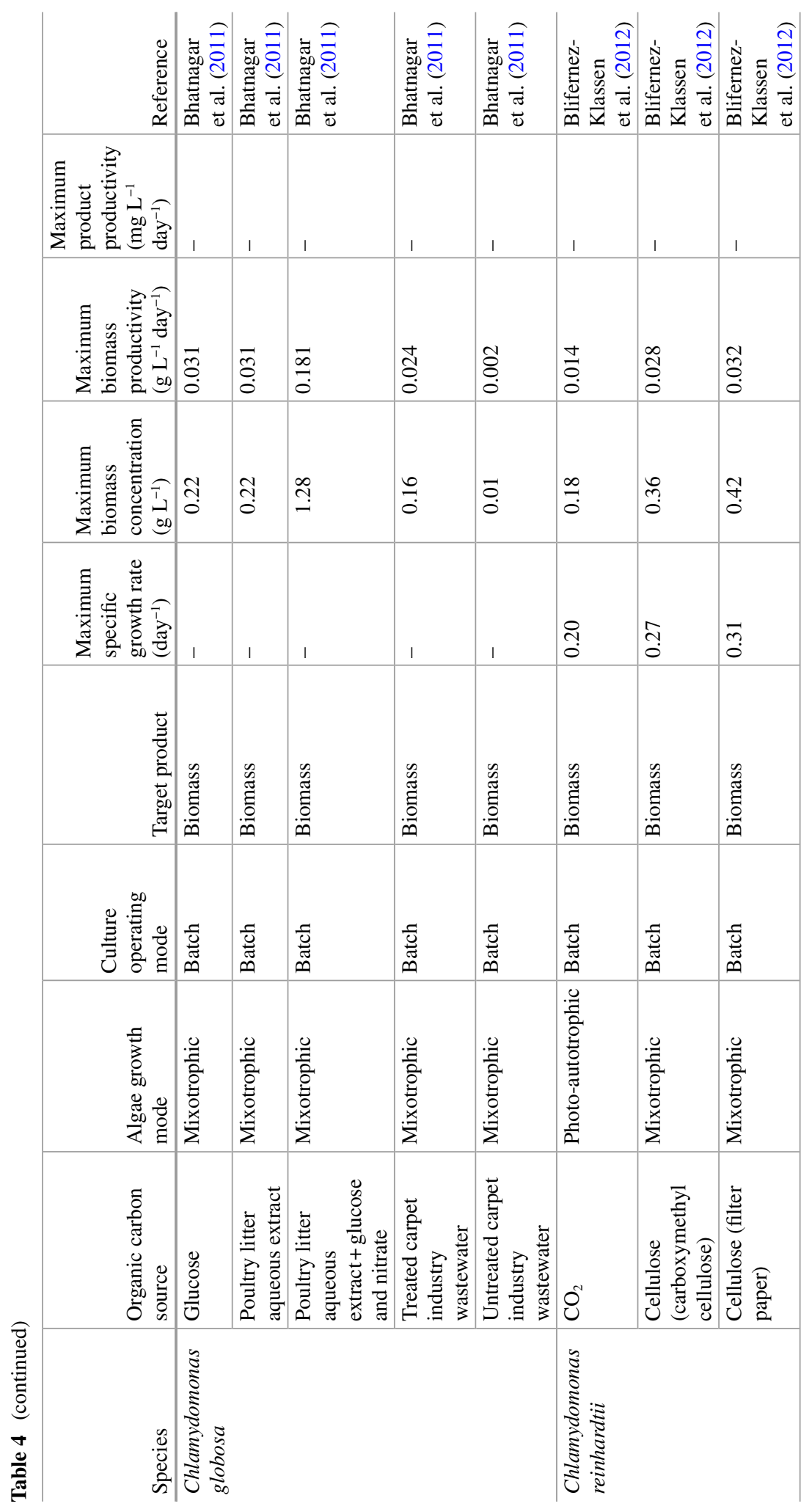




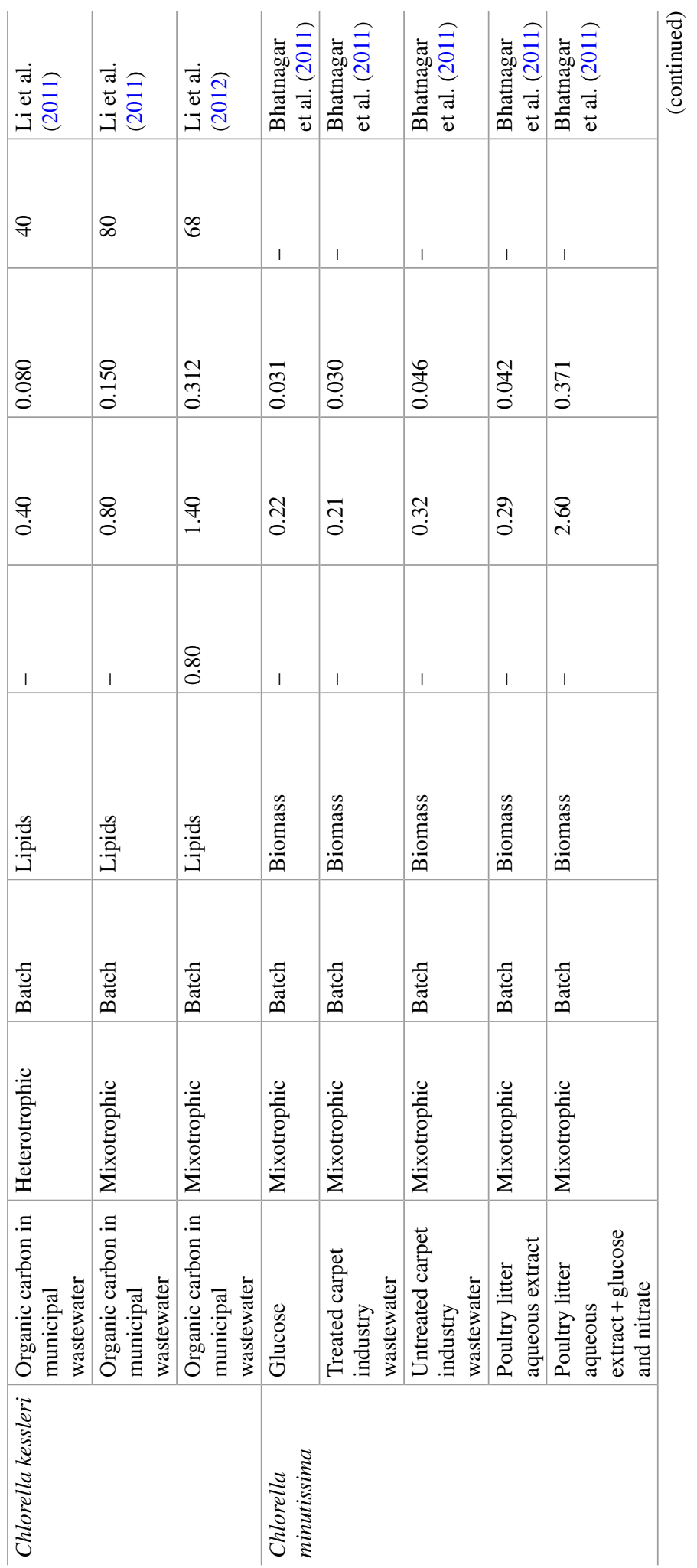




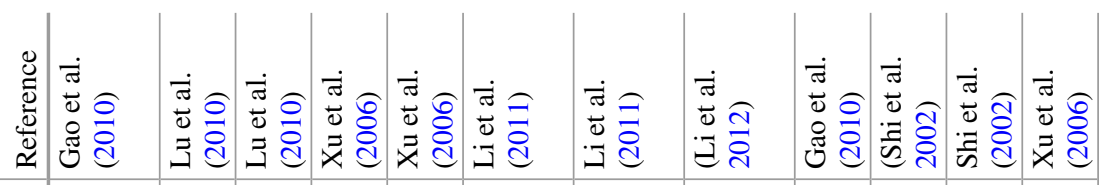

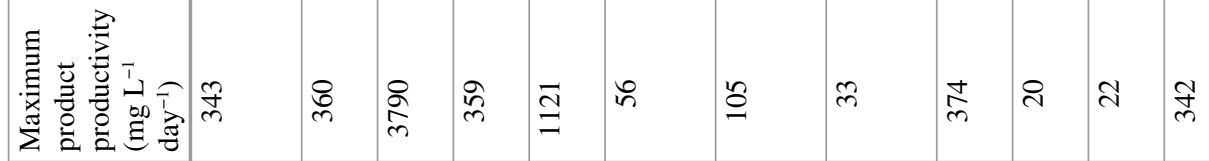

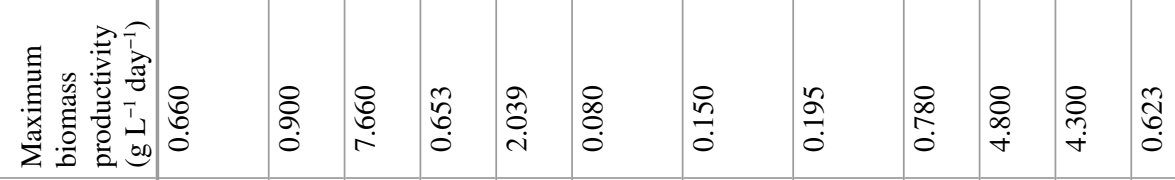

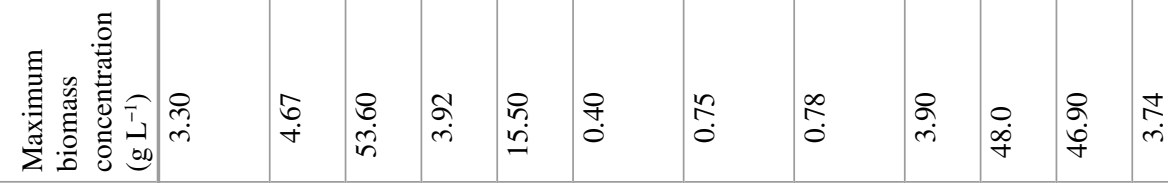

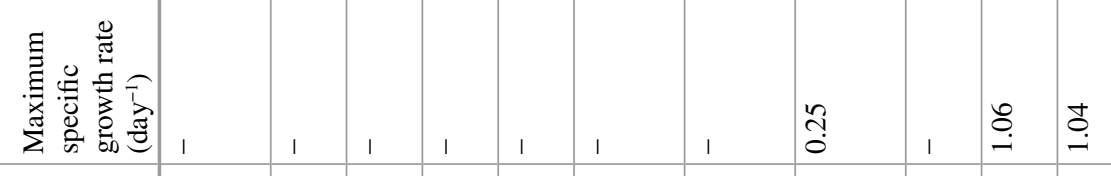

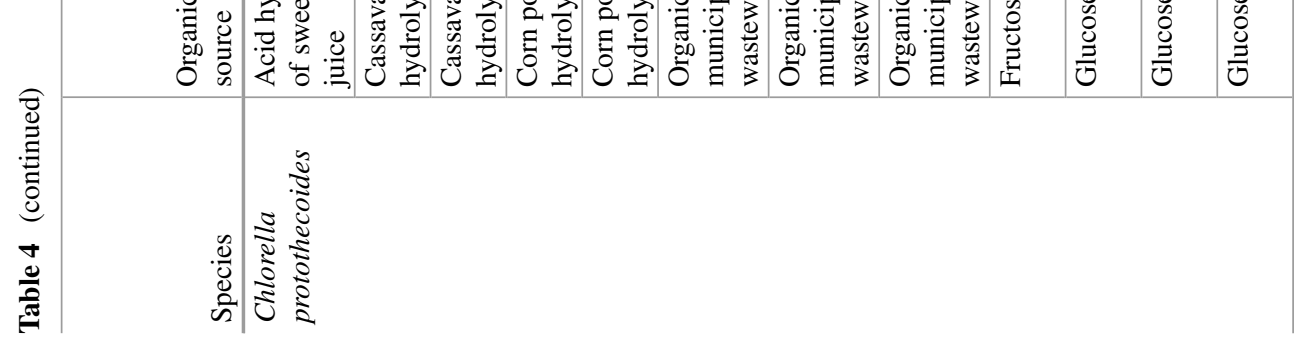




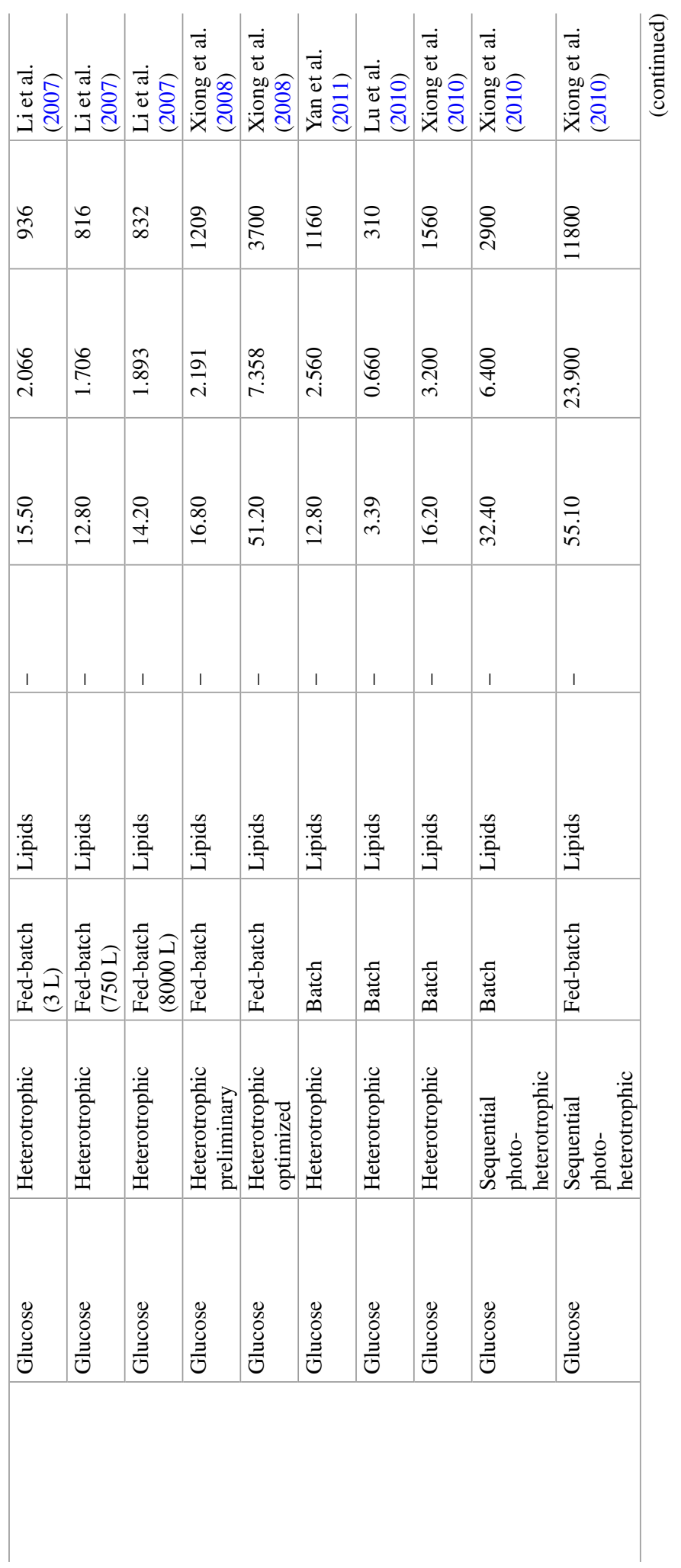




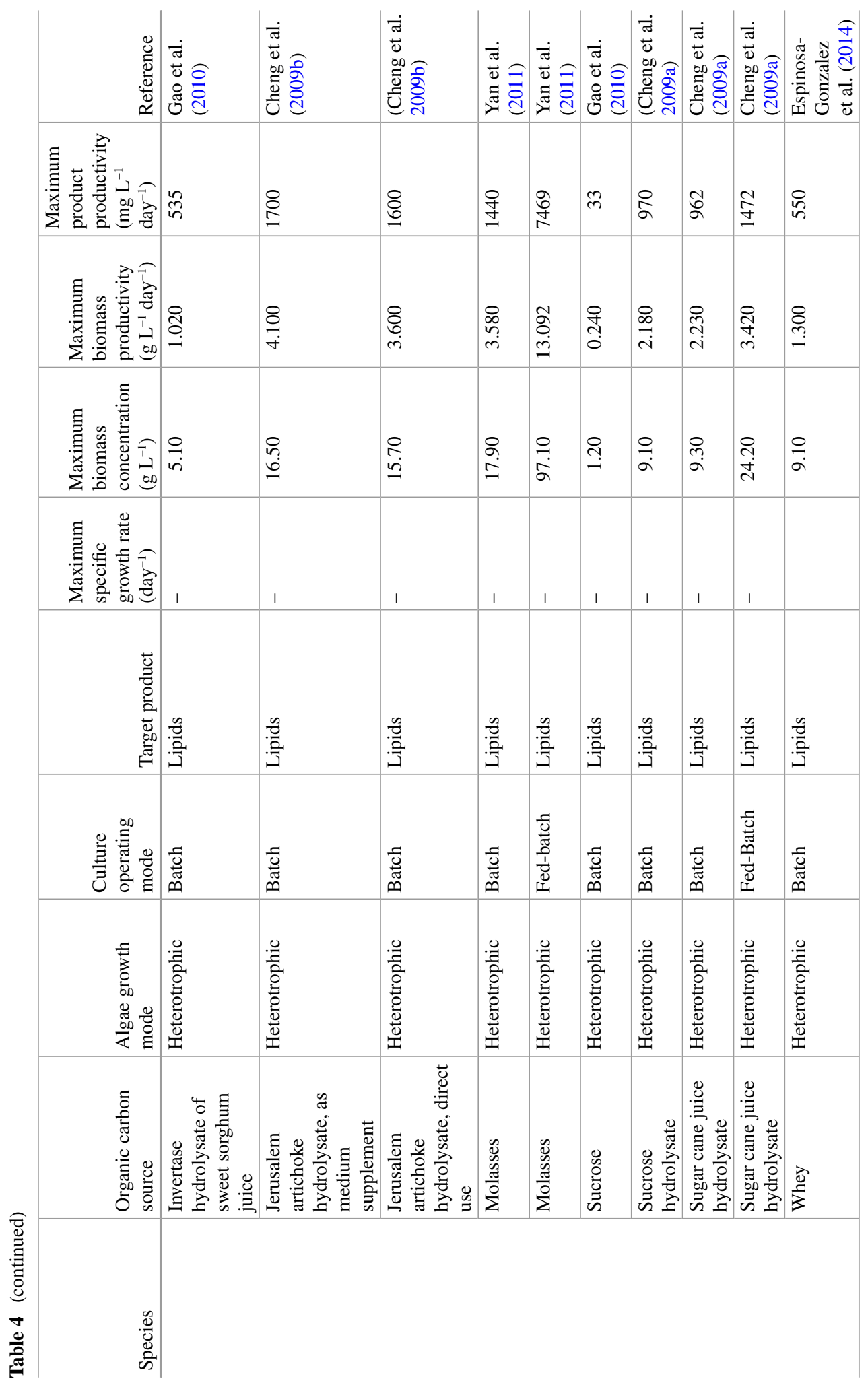




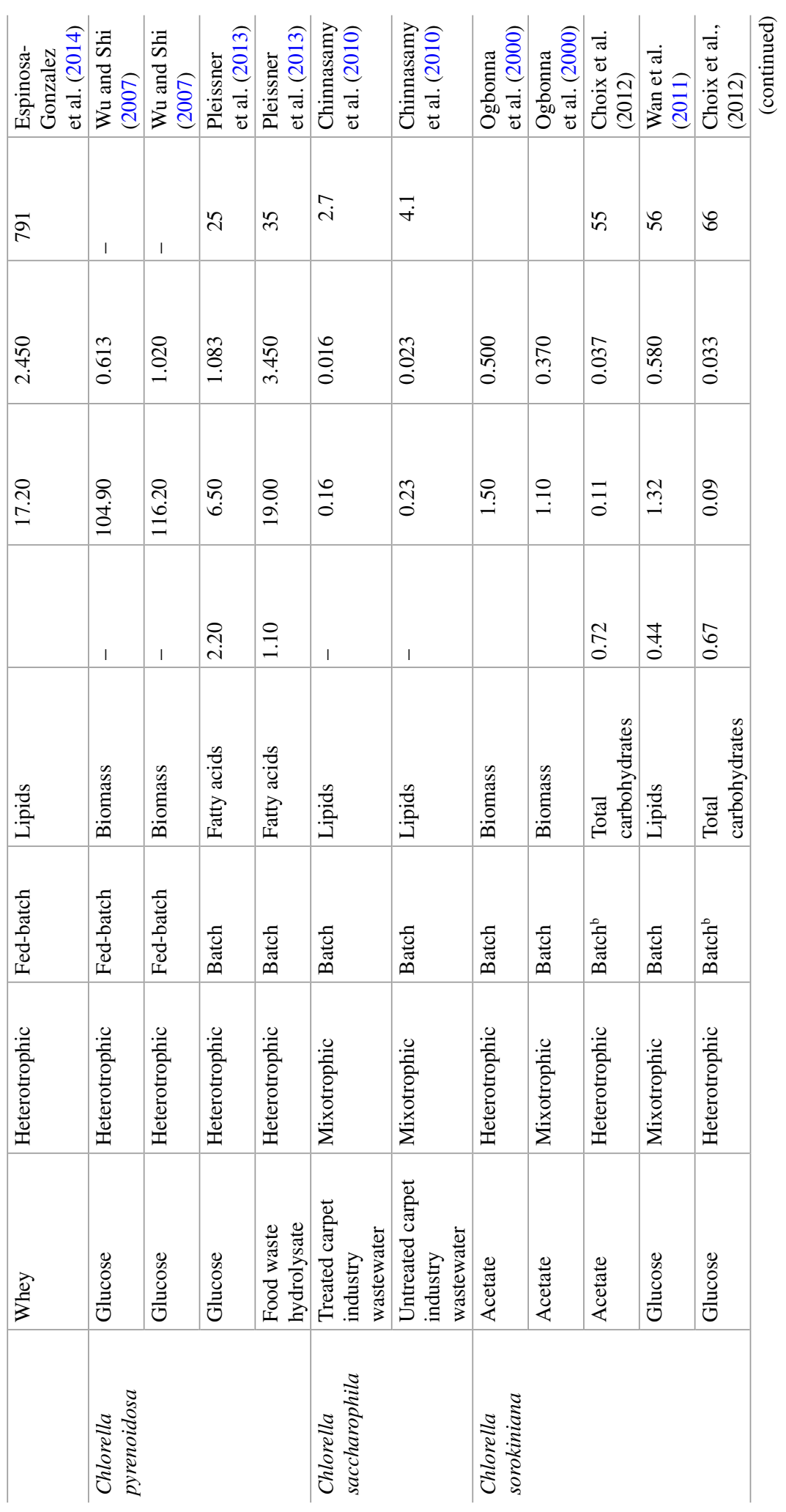




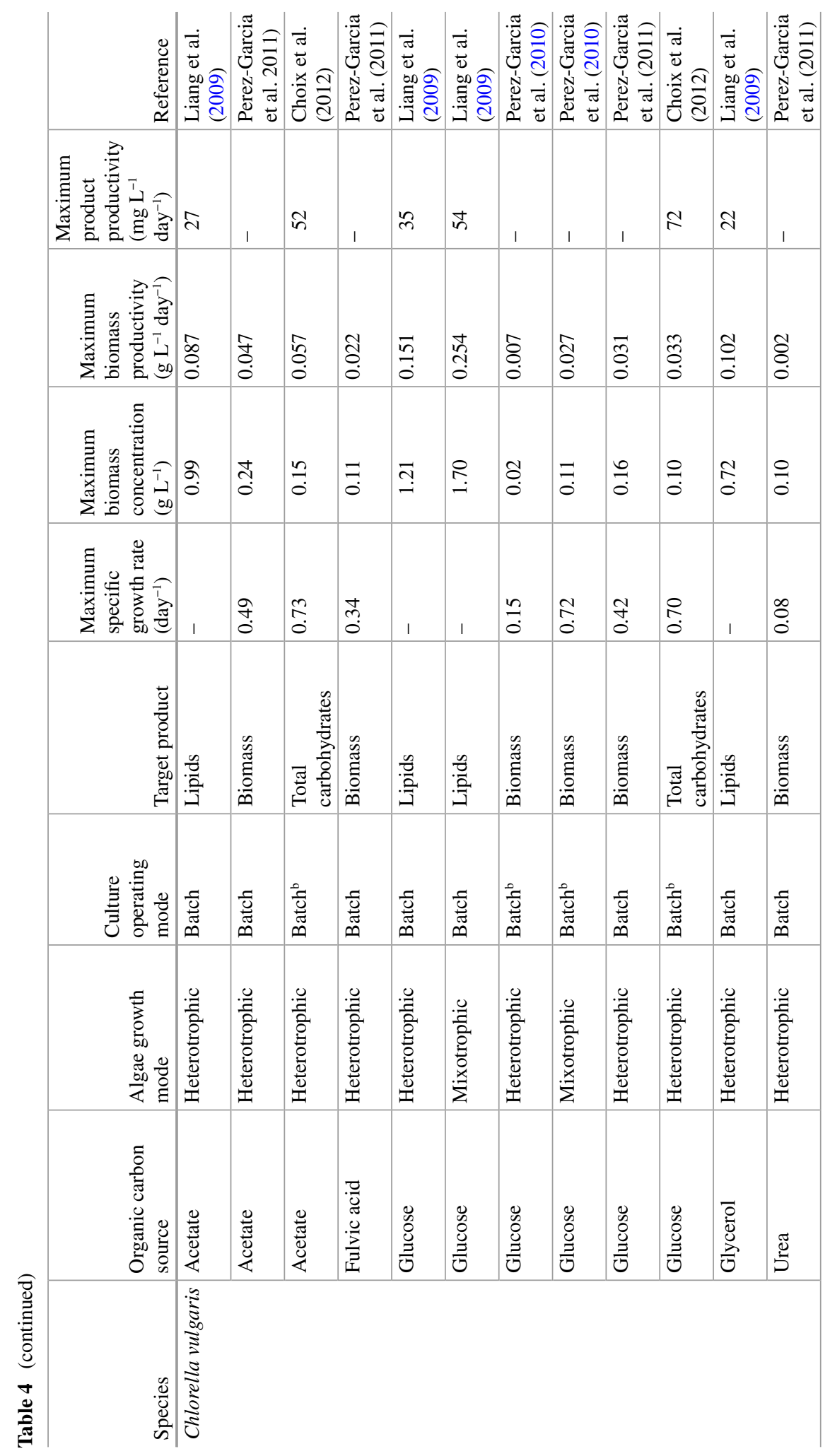




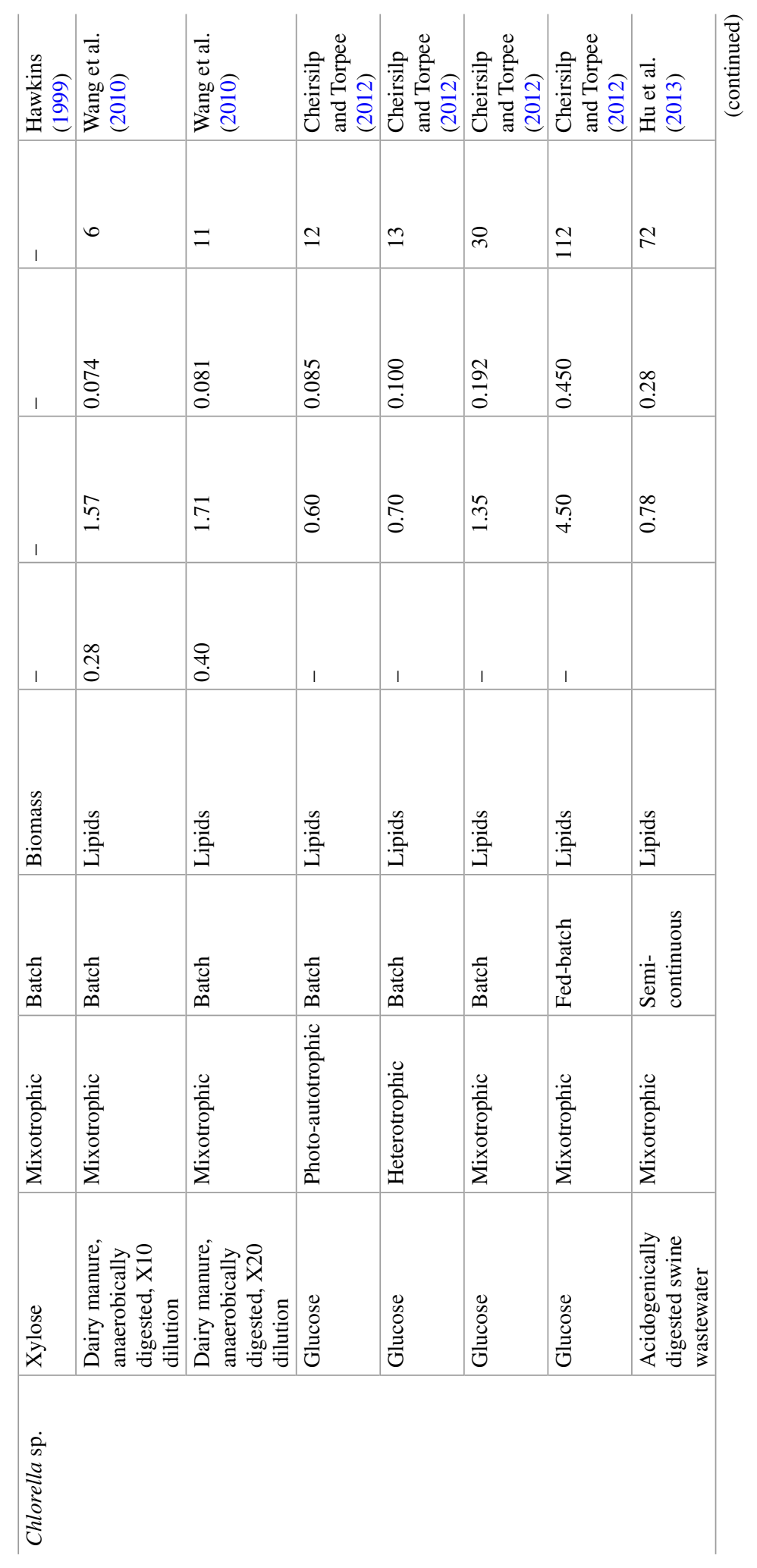




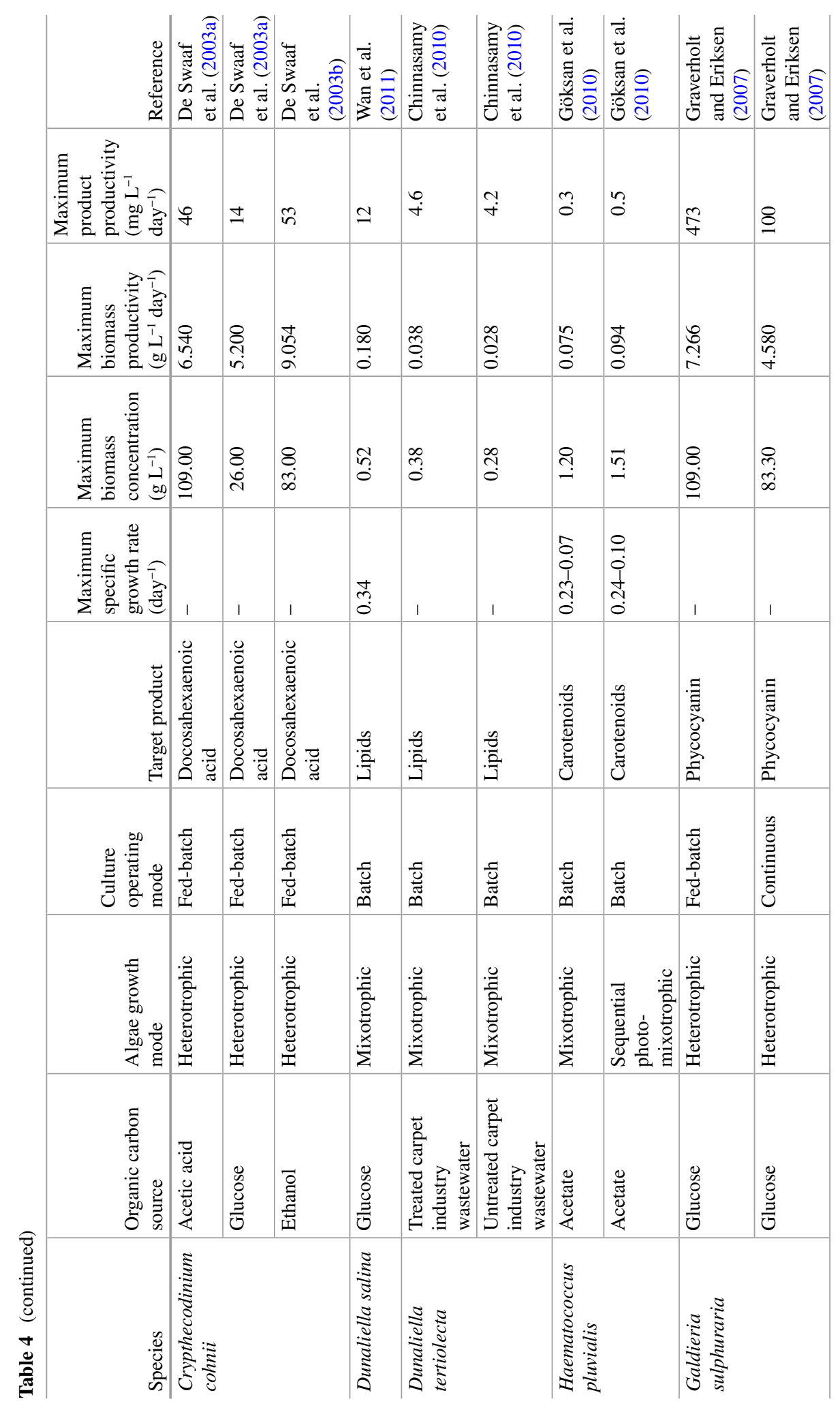




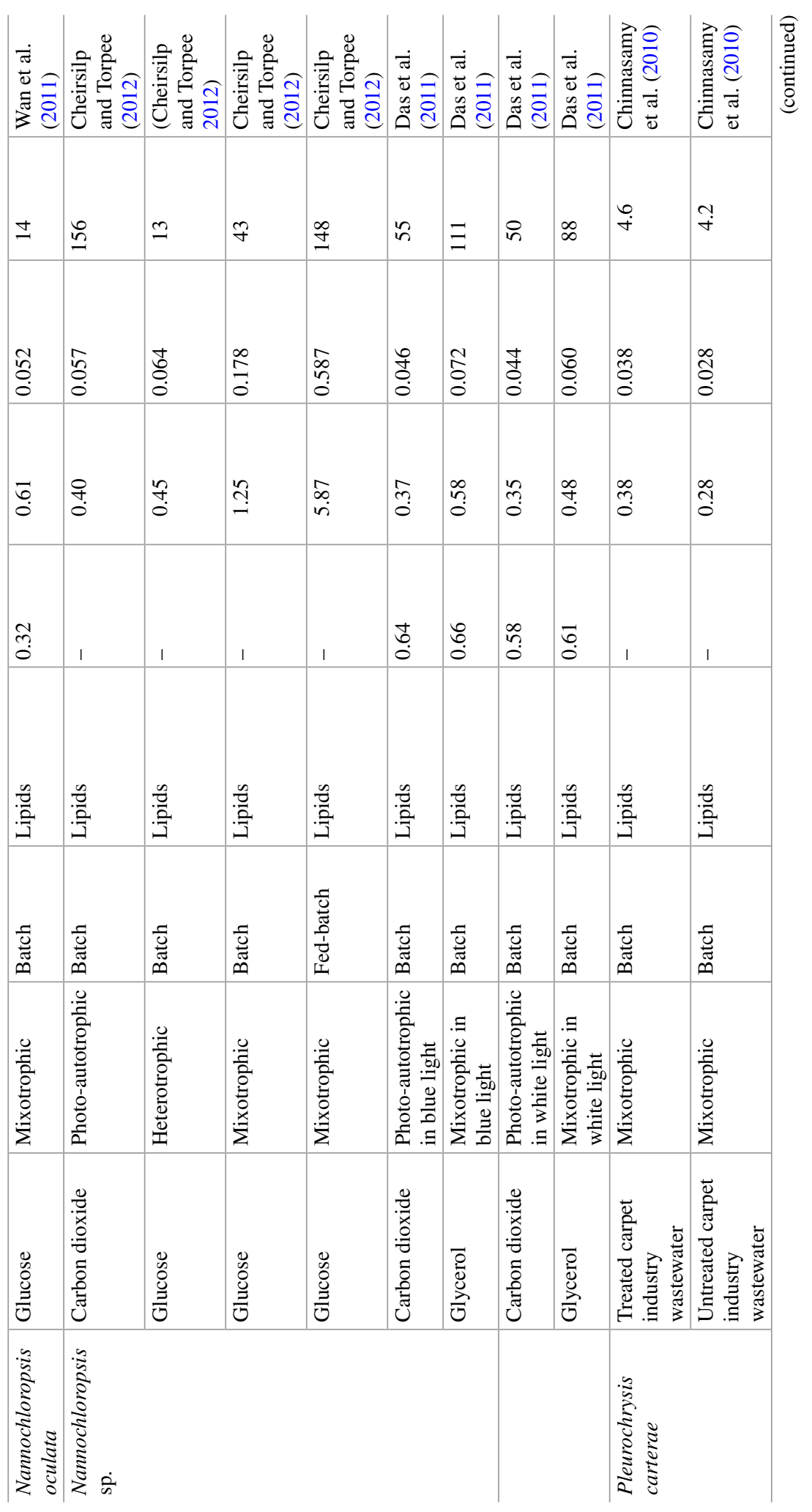




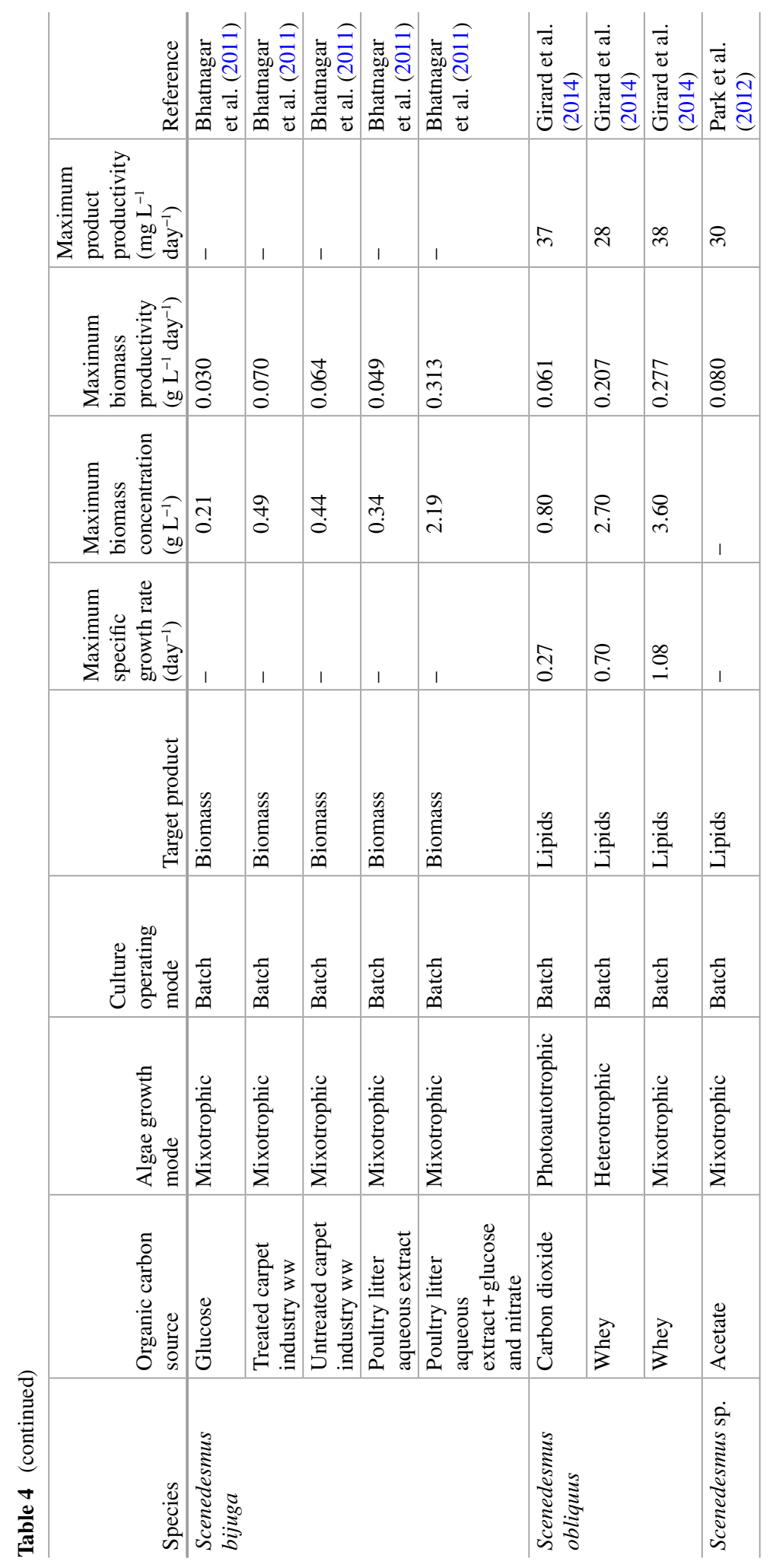




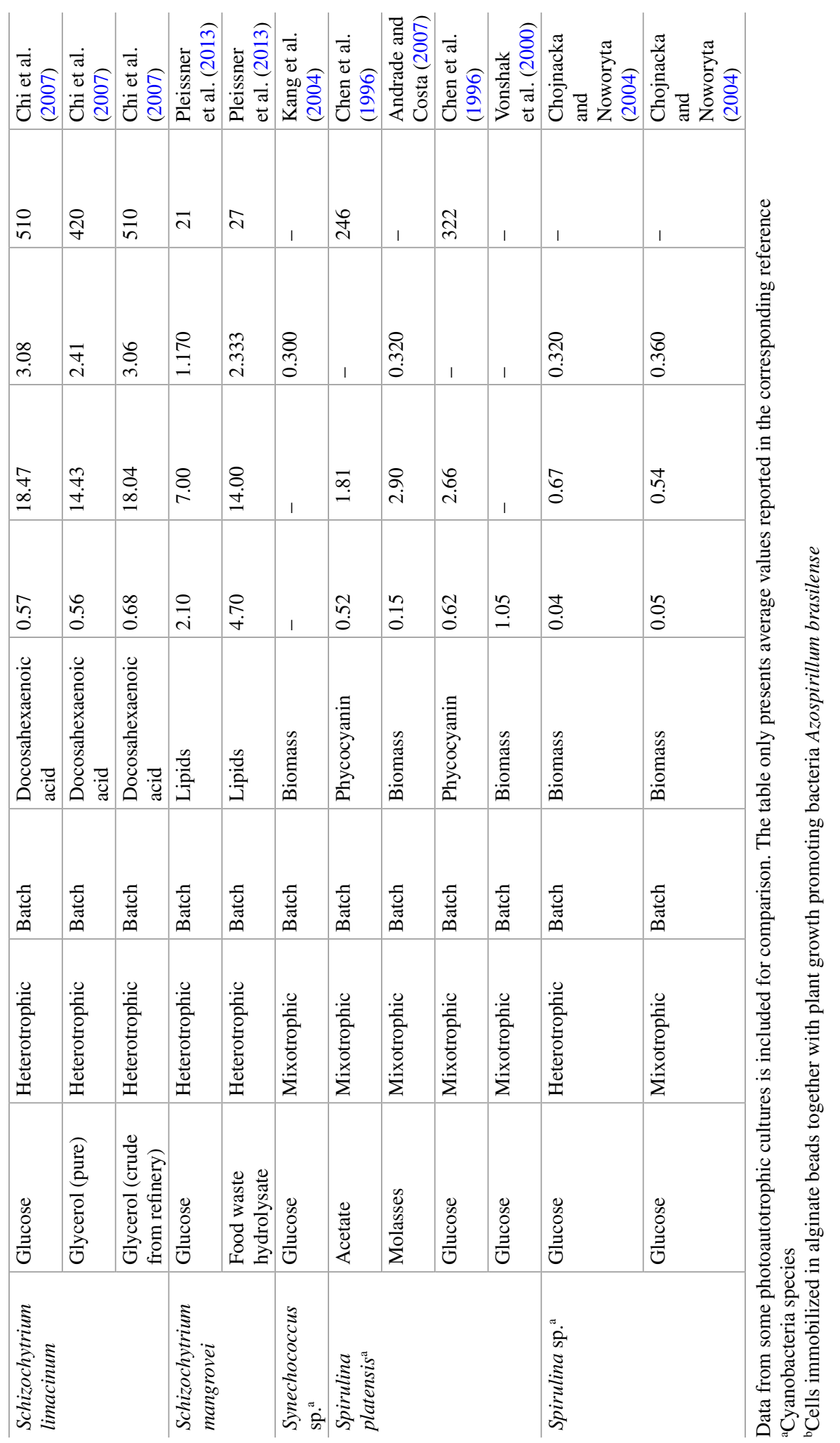


Chlamydomonas globosa, Scenedesmus bijuga, and Chlorella minutissima were able to grow mixotrophically and removed nitrogen and phosphorus far better in dark, colored, and opaque wastewaters rich in organic and inorganic nutrients than in the standard laboratory medium (Bhatnagar et al. 2011). Three strains of microalgae (Scenedesmus sp. ZTY2, Scenedesmus sp. ZTY3, and Chlorella sp. ZTY4) isolated from a domestic wastewater treatment plant were heterotrophically cultivated in real domestic wastewater with no illumination. The isolated strains contained good lipid content, from 55 to $80 \%$ of cell dry weight. Efficiencies of removal of dissolved organic carbon ranged from 55 to $65 \%$ (Zhang et al. 2013a). It is important to emphasize that under mixotrophic cultivations, microalgal consortia achieved higher biomass production than heterotrophic cultivations (Table 4).

New kinds of wastewater are being tested as potential sources of organic substrate for heterotrophic microalgal growth. For example, dairy manure contains levels of acetic and propionic acid that is readily available for microalgae cultivation (Wang et al. 2010). Also food waste hydrolysate has been used as culture medium and nutrient source for heterotrophic microalgae cultivation (Pleissner et al. 2013). Some wastewaters need a pre-treatment to make them available as carbon sources for microalgae cultivation. The pre-treatment eliminates side effects of solid particles and indigenous bacteria that may compete and outgrow the microalgae population. Large amounts of glucose, free amino nitrogen, and phosphate were recovered from food waste by fungal hydrolysis using Aspergillus awamori and A. oryzae. Schizochytrium mangrovei and Chlorella pyrenoidosa grew well on the recovered nutrients. Growth rate, biomass, and lipid production were higher in cultures cultivated on food waste hydrolysate than in control cultures growing on conventional medium with glucose. Impressively, at the end of the cultivation, 10-20 g of biomass, out of the original $100 \mathrm{~g}$ food waste (as dry weight) were produced and were rich in carbohydrates, lipids, proteins, and saturated and polyunsaturated fatty acids (Pleissner et al. 2013) (Table 4). In another example, cultivation of microalgae on swine manure proved to be a practical and economical organic substrate for production of algae feedstock (Hu et al. 2013). In their study, acidogenic-anaerobic digestion was used as a pre-treatment of fresh swine manure before it was used as the substrate in heterotrophic cultures of Chlorella sp. This yielded nutrient removal rates of $751.33 \mathrm{mg}$ COD L ${ }^{-1}$ day $^{-1}, 20.21 \mathrm{mg} \mathrm{PO}_{4}-\mathrm{P} \mathrm{L}^{-1}$ day $^{-1}$ and $60.39 \mathrm{mg}$ $\mathrm{NH}_{3}-\mathrm{N} \mathrm{L}^{-1}$ day $^{-1}$. At the same time, the experiment achieved lipid productivity of $3.63 \mathrm{~g} \mathrm{~m}^{2}$ day $^{-1}$ or $10 \times 10^{3} \mathrm{~L} \mathrm{ha}^{-1}$ year $^{-1}$ (Hu et al. 2013). In another experiment, wastewater produced from hydrothermal liquefaction of biomass was mixotrophically grown in mixed cultures of microalgae and bacteria. This wastewater had concentrations of COD from 50 to $130 \mathrm{~g} \mathrm{~L}^{-1}$, total nitrogen from 5 to $20 \mathrm{~g} \mathrm{~L}^{-1}$, ammonia from 3 to $12 \mathrm{~g} \mathrm{~L}^{-1}$ and phosphorus from 0.6 to $2 \mathrm{~g} \mathrm{~L}^{-1}$. Consequently, this wastewater needed to be diluted ( 100 times) to be used for microalgae heterotrophic/mixotrophic cultivation (Zhou et al. 2013).

Microalgae and cyanobacteria respond to many organic pollutants in various ways, such as growth inhibition, bioaccumulation, and biodegradation. Because of their versatile metabolism and their capacity to switch rapidly from one growth mode to another, mixotrophic microalgae can be successfully employed for remedi- 
ating polluted environments (Subashchandrabose et al. 2013). The ecological advantage of employing mixotrophic cyanobacteria and microalgae for bioremediation is that decreasing concentrations of organic pollutants will have no adverse effect on their growth. In contrast, if a heterotrophic regime is employed, no further biomass production can be expected after degradation of the organic pollutant. The dual-purpose process of chemical production-wastewater treatment, as a general strategy for many microalgal systems, though it looks very attractive, has many challenges, as discussed above. Therefore, with current knowledge, expectations should be cautious. With better strains and better technologies, it may be possible that we can achieve the microalgal potential and make the process efficient and cost effective (Liang 2013).

\subsection{Processed Materials}

To reduce costs for organic carbon sources, various processed materials (mostly wastes) have been examined. These feedstock include: Jerusalem artichoke hydrolyzed by inulinase (Cheng et al. 2009b), sweet sorghum hydrolyzed liquid by acid or invertase (Gao et al. 2010), cassava starch hydrolyzed by amylase and glucoamylase (Lu et al. 2010), molasses hydrolyzed by invertase (Yan et al. 2011), Na-acetate (Perez-Garcia et al. 2011a, b), crude glycerol (Das et al. 2011; Liang et al. 2009), corn powder hydrolysate (Xu et al. 2006), sugar cane juice hydrolysate (Cheng et al. 2009a), and cheese whey (Girard et al. 2014). A summary of these studies is presented in Table 4.

Food industry by-products and wastes are a promising feedstock for production of chemical compounds by heterotrophic microalgae. Molasses, a by-product of sugar refinery, is a cheap carbon source proven to be an excellent alternative to glucose in promoting microalgal lipid accumulation because it contains about $36 \%$ sucrose (Yan et al. 2011). Yet, pre-enzymatic hydrolysis by invertase was required for molasses to be suitable for growing microalgae. Microalgal biomass derived from medium with molasses hydrolysates was higher than a glucose medium. This may happen because molasses contains fructose, which is also a source of carbon. The biomass of $C$. protothecoides was $97.1 \mathrm{~g} \mathrm{~L}^{-1}$ and lipid production was as high as $5.5 \mathrm{~g} \mathrm{~L}^{-1} \mathrm{day}^{-1}$ in fed-batch cultures (Table 4). Yan et al. (2011) estimated that the cost of producing lipids from molasses was $50 \%$ of the cost of using a glucosebased medium.

Espinosa-Gonzalez et al. (2014) and Girard et al. (2014) proposed using cheese whey in mixotrophic microalgae cultures. Whey is generated in large quantities as a by-product of cheese production. Its main constituent is lactose $(>80 \% \mathrm{w} / \mathrm{w}$ of the total dissolved solids). Whey has the advantage that it is free of contaminants and can readily be used as a carbon source for microalgae cultivation (Girard et al. 2014). Substituting $40 \%$ (v/v) of microalgae culture medium with whey significantly stimulated growth of Scenedesmus obliquus. Consequently, yields of biomass under mixotrophic $\left(3.6 \mathrm{~g} \mathrm{~L}^{-1}\right)$ and heterotrophic $\left(2.7 \mathrm{~g} \mathrm{~L}^{-1}\right)$ conditions were 
significantly greater than under photo-autotrophic conditions $\left(1.2 \mathrm{~g} \mathrm{~L}^{-1}\right)$. Crude glycerol, a large volume by-product of the biodiesel industry, has been successfully used as an alternative substrate for growing Schizochytrium limacinum (Chi et al. 2007) and Chlorella vulgaris (Liang et al. 2009). Using glycerol, heterotrophic cultivation of $S$. limacinum reached a biomass concentration of $18 \mathrm{~g} \mathrm{~L}^{-1}$ and production of docosahexaenoic acid of $510 \mathrm{mg} \mathrm{L}^{-1}$ day $^{-1}$ (Table 4; Chi et al. 2007).

Microalgae cells do not assimilate complex sugars, such as polysaccharides; therefore, carbon sources, such as vegetable powders rich in polysaccharides, need to be hydrolyzed to break the compounds into di- and mono-saccharides, which can be assimilated by microalgae cells. Xu et al. (2006) optimized microalgae growth on hydrolyzed corn powder, using $\alpha$-amylase and glucoamylase. This hydrolysate led to high yields of $C$. protothecoides biomass $\left(15.5 \mathrm{~g} \mathrm{~L}^{-1}\right)$ in batch culture (Table 4). C. protothecoides also grew well on medium supplemented with sugar cane juice hydrolysate but not on raw sugar cane juice (Cheng et al. 2009a). The sugar cane juice hydrolysate contained $18.5 \%$ sucrose and $1.7 \%$ other fermentable sugars, such as glucose and fructose, and led to a biomass concentration of $25.4 \mathrm{~g} \mathrm{~L}^{-1}$ (Cheng et al. 2009a). Sweet sorghum hydrolysate liquid also promoted heterotrophic growth of $C$. protothecoides, leading to a biomass concentration of $5.1 \mathrm{~g} \mathrm{~L}^{-1}$ (Gao et al. 2010). Raw sweet sorghum juice contains sucrose, fructose, and glucose (101.7, 33.1, and $25.0 \mathrm{~g} \mathrm{~L}^{-1}$, respectively). However, sucrose cannot be used by heterotrophic $C$. protothecoides; it must be hydrolyzed to glucose and fructose prior to use as a feedstock. Finally, Lu et al. (2010) cultivated $C$. protothecoides heterotrophically, using cassava hydrolysate powder, which led to an impressive biomass concentration of $53 \mathrm{~g} \mathrm{~L}^{-1}$ in fed-batch cultures (Table 4).

\subsection{Lignocellulose Material}

Lignocellulose biomass is a carbon-neutral $\left(\mathrm{CO}_{2}\right.$ is not emitted during its production), renewable, and sustainable source of organic carbon. Sugars released from this feedstock are inexpensive carbon sources for heterotrophic cultivation of microalgae. While this sounds promising, this carbon source has the following limitations: (1) Pretreatment to break down lignocellulose, such as wood chips or plant fibers, to simple sugars is expensive; (2) By-products from the breakdown are numerous, roughly classified as aliphatic acids, aromatic acids, and aldehydes/ketones. Some of those products may cause inhibition or toxicity to microalgae. Though the options of lignocellulosic material are many, the detoxification processes increase costs; and (3) The major monomeric sugars in cellulosic feedstock are glucose and xylose. Most microalgal species thrive on glucose, but species that can use xylose are few and perhaps limited only to the genus Chlorella (Liang 2013). So far, only one Chlorella strain used xylose mixotrophycally or when glucose was present in the media (Hawkins 1999). In the dark or with the absence of glucose, this strain did not prosper on xylose. He speculates that a cofactor or other metabolic pathways is necessary 
to metabolize xylose and that energy or cofactors derived from glucose or light metabolism can initiate the xylose utilization pathway.

Recently, cellulose as a carbon source was used by the microalgae Chlamydomonas reinhardtii (Blifernez-Klassen et al. 2012), an ability which had previously been known for degrading cellulose by non-photosynthetic organisms. This opens new prospects for using cellulose waste in microalgae cultivation. In the presence of light, $C$. reinhardtii used cellulose for growth in the absence of other carbon sources by secreting cellulolytic enzymes (endo- $\beta$-1,4-glucanases), when the medium was supplemented with carboxymethyl cellulose or filter paper (Table 4). During this mixotrophic regime, it was impossible to discriminate between the contributions of photosynthesis and heterotrophic assimilation of cellulose on cell growth. This cellulose degrading and assimilation phenotype was not observed in the closely related microalga C. kessleri (Blifernez-Klassen et al. 2012).

Research in this line should focus on developing cheap, effective, and inexpensive pretreatment techniques, eliminate inhibitory by-products or decrease their concentrations. Considering the many publications and enormous efforts dedicated to biomass pretreatment in recent times, it is reasonable to expect that pretreatment methods will be developed (Liang 2013). For example, removal of degraded byproducts from biomass hydrolysates can be achieved by physical (vacuum evaporation), chemical (ion exchange or over-liming) and biological (enzymes or microorganisms that modify the composition of toxic compounds) methods. Finally, future research efforts should also focus on: (a) bio-prospecting of species that grow on xylose; (b) producing strains that use xylose by genetic and metabolic engineering; and (c) couple microalgae growth with other xylose, using microbial species in the same reactor or sequentially,

\section{Cultivation Methods}

\subsection{The Bioreactor}

Heterotrophic microalgae are grown in stirred tank bioreactors (STRs), commonly known as fermenters. Because heterotrophic microalgae growth is independent of light energy, much simpler scale-up possibilities are available since a smaller reactor surface-to-volume ratio is used compared with photo-bioreactors (Brennan and Owende 2010). Table 5 summarizes the main technical aspects of cultivation systems for each microalgae growth regime. Fermenters provide a high degree of growth control and also lower harvesting costs because higher cell densities are possible, compared with photo-bioreactors and open ponds. Also, bioreactor set-up costs are lower than for closed photo-bioreactors. Heterotrophic algal growth requires oxygen, mixing, temperature control, and nutrients in a sterile environment. Therefore additional expenses associated to facilities for preparation of growth medium and reactor sterilization must be considered. The 
Table 5 Technical aspects for cultivation at different microalgae growth modes

\begin{tabular}{|c|c|c|c|}
\hline Technical aspect & Photo-autotrophic & Heterotrophic & Mixotrophic \\
\hline Energy source & Light & Organic carbon & $\begin{array}{l}\text { Light and organic } \\
\text { carbon }\end{array}$ \\
\hline Carbon source & Inorganic carbon & Organic carbon & Inorganic and organic \\
\hline $\begin{array}{l}\text { Use of renewable energy } \\
\text { source }\end{array}$ & Yes & Not in principle & Partially \\
\hline $\begin{array}{l}\text { Biomass/compounds } \\
\text { productivity }\end{array}$ & Low & Medium & High \\
\hline $\begin{array}{l}\text { Light availability } \\
\text { requirement }\end{array}$ & Obligatory & Not required & Not obligatory \\
\hline Limiting factor for growth & Light & Oxygen & Light and oxygen \\
\hline $\mathrm{CO}_{2}$ emission & $\begin{array}{l}\text { Negative emission, } \\
\text { sink }\end{array}$ & $\begin{array}{l}\text { Positive } \\
\text { emission }\end{array}$ & $\begin{array}{l}\text { Neutral, } \mathrm{CO}_{2} \text { produced } \\
\text { and consumed }\end{array}$ \\
\hline Bioreactor type & $\begin{array}{l}\text { Photobioreactor. } \\
\text { Open or close in } \\
\text { panels or tubes }\end{array}$ & Fermenters & $\begin{array}{l}\text { Photo-bioreactor. Open } \\
\text { or close in panels or } \\
\text { tubes }\end{array}$ \\
\hline $\begin{array}{l}\text { Bioreactor's vessels } \\
\text { availability }\end{array}$ & In house crafted & $\begin{array}{l}\text { Commercially } \\
\text { available }\end{array}$ & In house crafted \\
\hline $\begin{array}{l}\text { Bioreactor's surface to } \\
\text { volume ratio }\left(\mathrm{m}^{2} / \mathrm{m}^{3}\right)\end{array}$ & High & Low & High \\
\hline $\begin{array}{l}\text { Control of operation } \\
\text { parameters in bioreactors }\end{array}$ & High & High & High \\
\hline Sterility & Usually sanitized & Sterility required & $\begin{array}{l}\text { Sterility preferred but } \\
\text { not required }\end{array}$ \\
\hline Contamination risk & Low & High & Medium \\
\hline Harvesting difficulty & $\begin{array}{l}\text { High due diluted } \\
\text { biomass } \\
\text { concentration }\end{array}$ & $\begin{array}{l}\text { Low due dense } \\
\text { biomass } \\
\text { concentration }\end{array}$ & $\begin{array}{l}\text { Low due dense } \\
\text { biomass concentration }\end{array}$ \\
\hline Bioreactor set up cost & $\begin{array}{l}\text { High per unit of } \\
\text { volume }\end{array}$ & $\begin{array}{l}\text { Low per unit of } \\
\text { volume }\end{array}$ & $\begin{array}{l}\text { High per unit of } \\
\text { volume }\end{array}$ \\
\hline Bioreactor operation $\cos ^{\mathrm{a}}$ & $\begin{array}{l}\text { Low per } \mathrm{kg} \text { of } \\
\text { biomass }\end{array}$ & $\begin{array}{l}\text { Medium per kg } \\
\text { of biomass }\end{array}$ & High per kg of biomass \\
\hline
\end{tabular}

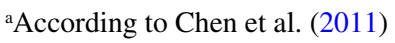

growth medium and pipelines for inoculation, feeding substrate, and sampling are commonly sterilized by autoclave. With heterotrophic growth, oxygen for respiration is the main limiting factor; therefore, the bioreactor's oxygen supply and good medium mixing are essential.

A significant advantage of heterotrophic cultivation is that STRs are easily scalable up to commercial size (with working volumes of $\sim 10,000 \mathrm{~L}$ ) and that vessels are available commercially for cultivation of other microorganisms (Barclay et al. 2013; Li et al. 2007). Producing lipids by heterotrophic $C$. protothecoides were successfully scaled up from $250 \mathrm{~mL}$ flasks to STRs with net volume of 5, 750, and 11,000 L ( $\mathrm{Li}$ et al. 2007). In pilot (750 L) and commercial-scale (11,000 L) bioreactors, real-time respiration-rate monitoring used a dissolved oxygen online detector, and the consumption of substrates was monitored by manual sampling. Inoculation 

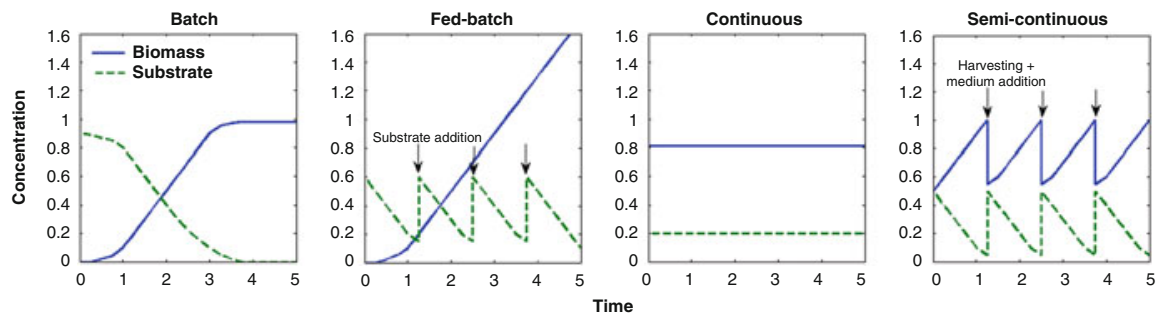

Fig. 5 Typical concentration profiles observed in microalgae cultures under different operation modes. Concentration profiles are similar to those observed in bacteria and fungi fermentations

was performed in three steps: the pre-inoculum $C$. protothecoides cells were first grown in $100 \mathrm{~L}$ of medium, then in two $750 \mathrm{~L}$ STRs with $400 \mathrm{~L}$ of medium each, and finally inoculated in 7,200 L of sterilized medium, with the final work volume reaching about $8,000 \mathrm{~L}$ in a $11,000 \mathrm{~L}$ STR. While the scaling up did not change the relative fatty acid composition of the biomass as cultivation was scaled up, total lipid content was slightly different $(46.1 \%, 48.7 \%$, and $43 \%$ of the cell dry weight) in samples from $5 \mathrm{~L}, 750 \mathrm{~L}$, and 11,000 L bioreactors, respectively ( $\mathrm{Li}$ et al. 2007).

The key practical issues required for large-scale heterotrophic cultivation of microalgae are: (a) Good survival of the strain during cultivation; (b) The strains need to be genetically robust and stable under production circumstances and resistant to mutations and infections by other microorganisms; (c) Ideally, strains should be able to grow under extreme conditions, such as high or low $\mathrm{pH}$, high temperatures, or high salinity; (d) Overall low cultivation costs based on the strain to efficiently use inexpensive, common carbon sources, tolerate environmental changes, and generate economic value by generating large quantities of the metabolite(s); and, (e) At the industrial level, the strains must be easy to handle; its cell walls must withstand hydrodynamic and mechanical shear occurring in large bioreactors and it should produce high density biomass, all in minimally modified fermenters used for other microorganisms (Chen and Chen 2006; Day et al. 1991; Wijffels et al. 2013).

\subsection{Mode of Culture Operation}

Heterotrophic and mixotrophic cultures in fermenters or photo-bioreactors can be operated in batch, fed-batch, continuous, and semi-continuous modes (Table 4). Figure 5 illustrates ideal profiles for biomass and substrate concentration observed in fermenters operated in these modes. Batch mode fermentation is commonly adopted for growing heterotrophic microalgae. It achieves biomass concentrations from 0.5 to $20 \mathrm{~g} \mathrm{~L}^{-1}$. For example, heterotrophic cultures of Chlorella pyrenoidosa on food waste hydrolysate reached $19 \mathrm{~g} \mathrm{~L}^{-1}$ (Pleissner et al. 2013). Batch mixotrophic cultures reached biomass concentrations of $3.6 \mathrm{~g} \mathrm{~L}^{-1}$, using Scenedesmus obliquus growing on whey (Girard et al. 2014). Fed-batch operation can reach 
higher biomass than batch mode. Heterotrophic fed-batch cultures of Chlorella pyrenoidosa and Crypthecodinium cohnii have reached the remarkable concentrations of $116 \mathrm{~g} \mathrm{~L}^{-1}$ and $109 \mathrm{~g} \mathrm{~L}^{-1}$ biomass growing on glucose and acetate, respectively (De Swaaf et al. 2003a; Wu and Shi 2007). Mixotrophic fed-batch cultures of Nannochloropsis sp. reached concentrations of $5.8 \mathrm{~g} \mathrm{~L}^{-1}$ (Cheirsilp and Torpee 2012). The advantage of the fed-batch mode lies in minimal restrictions by maintaining continuous feeding of the nutrient stream. Hence, microbial cells undergo log-phase growth during almost the whole cultivation cycle (Xiong et al. 2008). It appears that continuous (chemostate) and semi-continuous cultivation of heterotrophic and mixotrophic microalgae have been used in only a few studies (Graverholt and Eriksen 2007; Hu et al. 2013). During "chemostat" operations, the fermenter maintains steady chemical conditions. In continuous operation, fresh medium is continuously added to the bioreactor and old culture medium is continuously removed, both at a specific rate $\left(Q ;\right.$ in $\left.\mathrm{L} \mathrm{s}^{-1}\right)$. By changing the $Q$ value, the growth rate of microalgae is controlled. Continuous cultures can maintain a constant low substrate concentration and avoid, in practical and easy ways, adverse effects on growth and yield of end products (Graverholt and Eriksen 2007). In semi-continuous mode, a proportion of a batch culture is replaced with fresh medium when the majority of microalgae reach the late logarithmic growth phase. Then, the culture is maintained under batch operation for additional time to increase cell density before the next replacement of medium. The repeated harvesting-regrowing process can be maintained for weeks or several months without apparent decline in growth. In a semi-continuous process, the ratio between the daily-replaced and the total culture volumes defines the hydraulic retention time (HRT), which is a key parameter to control the rate of algae growth and nutrient uptake (Hu et al. 2013).

Sequential regime (two-stage regime) involves first exposing the culture to a photo-autotrophic period, followed by a heterotrophic or mixotrophic period. This strategy increased biomass and product concentrations of microalgal cultures. Xiong et al. (2010) separated the cultivation process into two independent but sequential phases, the "green" stage (nitrogen-sufficient and photosynthetic cultivation) and the "yellow" stage (nitrogen-deficient under heterotrophic growth for accumulating lipids). This regime was more efficient than mixotrophic cultivation alone. $C$. protothecoides cultures under sequential regime reached an impressive biomass concentration above $120 \mathrm{~g} \mathrm{~L}^{-1}$ and a lipid concentration above $40 \mathrm{~g} \mathrm{~L}^{-1}$ (Table 4). The average biomass and lipid productivities during the mixotrophic phase alone were 23.9 and $11.8 \mathrm{~g} \mathrm{~L}^{-1}$ day $^{-1}$, respectively. In another study, production of carotenoids by Haematococcus pluvialis increased $\sim 60 \%$ in cultures using a sequential regime of photo-autotrophic growth period (11 days), followed by a mixotrophic period (5 days), using supplements of acetate. This is in contrast with traditional mixotrophic cultivation, where acetate was added to the medium before inoculation (Göksan et al. 2010; Table 4). Yen and Chang (2013) studied growth of C. vulgaris under sequential photo-mixotrophic regimes. The results of the sequential regime reached a biomass of $\sim 64 \%$ over simple photo-autotrophic cultivation. During the mixotrophic stage, the low light intensity limited mixotrophic growth. This indicated that photosynthesis still plays an important role during mixotrophic 
cultivation. Finally, Zheng et al. (2012) proposed that high density heterotrophic cultivation of $C$. sorokiniana could be used as seed culture for subsequent photoautotrophic cultivation on a large scale.

\subsection{Culture Medium}

The composition of the basic culture medium for heterotrophic cultivation is similar to autotrophic cultivation with the sole exception of adding an organic carbon source. The culture medium BG11 has been widely used in photo-autotrophic, heterotrophic, and mixotrophic cultivation of microalgae (Bhatnagar et al. 2011; Cheirsilp and Torpee 2012; Chinnasamy et al. 2010; Göksan et al. 2010; Li et al. 2012; Wan et al. 2011). Also, the medium base supplemented with carbon sources and reduced glycine concentration has been widely used for heterotrophic growth of C. protothecoides (Miao and Wu 2006; Shi et al. 2002; Xu et al. 2006). The composition of the commonly used medium base is: $\left(\mathrm{g} \mathrm{L}^{-1}\right) 0.7 \mathrm{KH}_{2} \mathrm{PO}_{4}, 0.3 \mathrm{~K}_{2} \mathrm{HPO}_{4}, 0.3$ $\mathrm{MgSO}_{4} \bullet 7 \mathrm{H}_{2} \mathrm{O},\left(\mathrm{mg} \mathrm{L}^{-1}\right) 3 \mathrm{FeSO}_{4} \cdot 7 \mathrm{H}_{2} \mathrm{O}, 0.01$ vitamin $\mathrm{B} 1,1 \mathrm{~mL} \mathrm{~L}^{-1} \mathrm{~A} 5$ trace mineral solution; $1-2 \mathrm{~g} \mathrm{~L}^{-1}$ glycine or 1-7 $\mathrm{g} \mathrm{L}^{-1}$ yeast extract can be added as a nitrogen source (Cheng et al. 2009a; Hillebrand and Sommer 1999; Xiong et al. 2008). As a general rule, microalgae growing in the dark should have a C:N:P mass ratio of 46.1:7.7:1 (Hillebrand and Sommer 1999).

An optimal concentration of organic carbon in a medium depends on the carbon source, the microalgae strain, and cultivation conditions. An optimal concentration of carbon in the medium for heterotrophic or mixotrophic growth ranges from 10 to $30 \mathrm{~g} \mathrm{~L}^{-1}$, depending on the type of supplied carbon compound. When glucose is used, highest growth rates of diverse microalgal species occur at concentrations from 7 to $15 \mathrm{~g} \mathrm{~L}^{-1}$ (Bhatnagar et al. 2011; Cheirsilp and Torpee 2012; Liang et al. 2009; Wan et al. 2011). Greater concentrations of biomass can be achieved at glucose concentration above $30 \mathrm{~g} \mathrm{~L}^{-1}$. However, the biomass produced per unit of sugar consumed significantly decreases (Xiong et al. 2008). In the case of molasses, a concentration of $30 \mathrm{~g} \mathrm{~L}^{-1}$ of reduced sugar in hydrolyzed molasses provided the optimal production of biomass and lipids in cultivation of $C$. protothecoides (Yan et al. 2011). It is important to consider that in batch cultures, when cell density reaches its maximum concentration in the stationary phase, a higher carbon supply does not lead to continuous growth and accumulation of product (Cheng et al. 2009a). Inhibition of growth occurs in some microalgae even when the carbon source was applied at a low concentration, as occurred in C. reinhardtii. Inhibition occurred when acetate was $>0.4 \mathrm{~g} \mathrm{~L}^{-1}$ (Cheirsilp and Torpee 2012). Under mixotrophic growth conditions, 10 and $20 \mathrm{~g} \mathrm{~L}^{-1}$ of glucose improved cell growth significantly compared with medium containing 50 and $100 \mathrm{~g} \mathrm{~L}^{-1}$ of glucose. At $100 \mathrm{~g} \mathrm{~L}^{-1}$ (glucose solution at $10 \% \mathrm{w} / \mathrm{v}$ ), the glucose inhibited the microalga. Similarly, in Crypthecodinium cohnii cultures, glucose concentrations above $25 \mathrm{~g} \mathrm{~L}^{-1}$ inhibited growth (De Swaaf et al. 2003a). Hence, inhibition is strain-dependent and needs to be determined for each strain and set of culturing conditions (Liang et al. 2009). 
According to Xiong et al. (2008), among three inorganic nitrogen sources (urea, potassium nitrate, and ammonium nitrate) and two organic nitrogen sources (glycine and yeast extract), yeast extract at a concentration of $1-4 \mathrm{~g} \mathrm{~L}^{-1}$ is the most suitable to provide high biomass and lipid yield in heterotrophic Chlorella spp. However, this nitrogen source is more expensive than the inorganic sources. In general, accumulation of lipids and carotenoids (astaxanthin and lutein) in microorganisms is stimulated by an excess of carbon and a limitation in one or more of the other nutrients, especially nitrogen (De Swaaf et al. 2003a; Leyva et al. 2014; Shi et al. 2002). For example, accumulation of microbial lipids is often a two-phase process. In the first phase, cells undergo exponential division. In the second phase, the growth rate decreases from a nutritional limitation and lipids accumulate. Cheng et al. (2009a) found the maximum lipid contents, conversion ratio of sugar-to-biomass, and conversion ratio of sugar-to-lipids are achieved at different carbon-to-nitrogen ratios $(\mathrm{C} / \mathrm{N})$. This indicates the critical importance of the $\mathrm{C} / \mathrm{N}$ ratio and nitrogen concentration for microalga growth and accumulation of lipids. Additionally, high concentrations of nitrogen $\left(>1 \mathrm{~g} \mathrm{~L}^{-1}\right)$ leads to low efficiency of sugar consumption. Typically, $\mathrm{C} / \mathrm{N}$ in growth medium of 10 is used for lipid production in cultures of Chlorella spp. However, optimal values have been reported to be as high as a $\mathrm{C} / \mathrm{N}$ of 26 (Cheng et al. 2009a). Nitrogen $\left(\mathrm{NO}_{3}-\mathrm{N}\right)$ concentrations in the growth medium, by itself, influences the amount of lipid contents and variety of fatty acids in Chlorella vulgaris and $C$. sorokiniana at a concentration from 2.5 to $5.6 \mathrm{mg} \mathrm{L}^{-1}$, as optimal to achieve maximum lipids production (Cha et al. 2011). Shen et al. (2010) tested the influence of various concentrations of urea, yeast extract, and nitrate for growing $C$. protothecoides. Among the tested conditions, the highest yield of lipids and biomass were obtained at the low concentration of $0.33 \mathrm{~g} \mathrm{~L}^{-1}$ of $\mathrm{NO}_{3}-\mathrm{N}$ medium. Further, urea has been used as a nitrogen source in heterotrophic cultures of C. protothecoides, where optimal production of lipids occurred at a concentration of $3.6 \mathrm{~g} \mathrm{~L}^{-1}$ (Shi et al. 2002).

It is important to consider that some organic substrates can change the $\mathrm{pH}$ of the medium and other properties such as viscosity and gas-liquid transfer coefficient. When sodium or potassium salt of acetate is used as a substrate, $\mathrm{pH}$ increases. This happens because the remaining $\mathrm{Na}^{+}$or $\mathrm{K}^{+}$couples with hydroxyl ions $\left(\mathrm{OH}^{-}\right)$or other anions to form alkalis. This phenomenon also occurs if reactors are neutralized with alkali. Since metallic hydroxides are stronger than organic acids, the media must be neutralized or at least brought to a non-inhibitory $\mathrm{pH}$ by adding an acid, for example, acetic acid into the cycle (Ratledge et al. 2001).

\subsection{Oxygen Supply}

Oxygen is a key factor in heterotrophic cultivation of microalgae. Independent of the organic substrate or the microalgae species, growth rates are enhanced by higher levels of aeration (Griffiths et al. 1960). Limited oxygen in a culture reduces 
the specific growth rate of microalgae cells, lowering biomass productivity when cell density is high (Wu and Shi 2007). For example, De Swaaf et al. (2003a) showed that high cell density of Crypthecodinium cohnii cultivated on acetic acid was a result of high oxygen demand. To maintain aerobic conditions, a high stirring speed had to be maintained during a large part of the process, hence, oxygen transfer is likely to be a limiting factor during a commercial scale cultivation of algae at high density.

\subsection{Mixing, Viscosity, and Foam}

Mixing is one of the most important operations when cultivating microalgae. Mixing within the fermenter is necessary for uniformly distributing nutrients and for gas exchange. Viscosity of the medium affects circulation, increasing with cell concentration or production of viscous cellular material. Many fermenters achieve adequate mixing with impellers and baffles (Behrens 2005; Dorian 1995). An alternative approach is based on air lifting. Fermenters with an internal plenum or draft tube have true airlifting capacity. Air is supplied on one side of the plenum or draft tube, creating a density difference between the side that is aerated and the side that is not. To be effective, airlifting usually requires a substantial airflow rate because sparging of air must supply all the energy for mixing and mass movement within the fermenter. High viscosity in cultures requires higher impeller speed or airflow, which increases power consumption and operational costs (Behrens 2005; Dorian 1995). Adding commercial polysaccharide-hydrolyzing enzymes decreases viscosity, which leads to an immediate increase in the concentration of dissolved oxygen, allowing a reduction in the speed of stirrers (De Swaaf et al. 2003a). Foam control is implemented as in yeast or bacterial fermentations. Usually, this involves monitoring foam production in the culture with sensors and using antifoam agents (De Swaaf et al. 2003a).

\subsection{Temperature}

The less efficient heat transfer in large-scale reactors may result in temperature problems from the combination of biological heat production and power input for mixing (De Swaaf et al. 2003a). Further, slightly differences in temperature can affect the metabolism of a given strain. For instance, maintenance of $C$. protothecoides culture in $30 \mathrm{~L}$ fed-batch cultures at $32{ }^{\circ} \mathrm{C}$ for $84 \mathrm{~h}$ resulted in a $19.9 \%$ increase in lutein content but a $13.6 \%$ decrease in cell dry weight concentration, compared with cultivation at $28{ }^{\circ} \mathrm{C}$. Greater lutein production results from a combination of limited nitrogen and high temperature stress (Shi et al. 2002). 


\subsection{Light in Mixotrophic Cultivation}

Mixotrophic cultivation is better when executed in closed photo-bioreactors (Bassi et al. 2014; Chen et al. 2011). These cultivation systems have transparent walls and vessel shapes (tubular and flat plate photo-bioreactors) that maximize the exposure of cells to light (Bassi et al. 2014). During mixotrophic growth, inorganic carbon is fixed through photosynthesis that is influenced by illumination conditions. Organic compounds are assimilated through aerobic respiration that is affected by availability. In mixotrophic cultivation, among all the environmental factors that affect the regime, the amount and quality of light are most important. Light not only affects microalgae photosynthesis, productivity, cell composition, and metabolic pathways, but also determines the economic efficiency of the cultivation process (Chen et al. 1996; Chojnacka and Noworyta 2004; Li et al. 2012). Light intensity profoundly affects mixotrophic growth of microalgae, independent of the carbon source. It is possible to control light intensity in photo-bioreactors by varying the number of fluorescent lamps, irradiance, and distance between the lamps and bioreactor's surface. The optimum light intensity for microalgae biomass and desired product production is not only strain-dependent, but also relies on the suitability of other factors of the environment, especially temperature and nutrient supply (Li et al. 2012).

Assimilation of carbon also modifies the cell's response to light. For example, as mentioned on Sect. 3 of this review, the maximum photosynthetic rate, light saturation constant, dark respiration rate, and light compensation point of mixotrophic cultures of the cyanobacteria Spirulina platensis were significantly higher than these rates in photo-autotrophic cultivation. As expected, mixotrophic cultures grew faster and reached higher biomass than photo-autotrophic cultivation. The growth rate of photo-autotrophic cultures was more sensitive to light. The differences between the two cultures were also apparent in their responses to exposure to extreme high intensities of light of $3000 \mu \mathrm{mol}$ photon $\mathrm{m}^{2} \mathrm{~s}^{-1}$. Although both cultures recovered from the stress of high photon flux density, the mixotrophic culture recovered faster and reached higher biomass concentration (Vonshak et al. 2000). Most species that are capable of mixotrophic growth need light. Usually the lower limit is $\sim 50 \mu \mathrm{mol}$ photon $\mathrm{m}^{2} \mathrm{~s}^{-1}$. These photons are not used for the photosynthetic cycles, but rather for enzyme activation for substrate assimilation (Chojnacka and MarquezRocha 2004; Chojnacka and Noworyta 2004). It is important to know, in advance, if a desired growth process is truly heterotrophic, mixotrophic, or photo-heterotrophic. If even a marginal amount of light is needed, this has to be considered within the reactor design, since light sources have to be evenly integrated, leading to rising costs or declining yields (Kröger and Müller-Langer 2011).

Light wavelength also affects microalgal productivity and product profile. For example, Nannochloropsis sp., was grown in phototrophic and mixotrophic conditions, with glycerol as the carbon source, under three primary monochromatic light wavelengths (red, green, and blue) and white light as the control. Lamps with lightemitting diodes (LEDs) were used to control light chromatic properties. The maximum specific growth rate was higher on blue $>$ white $>$ green $>$ red lights. The intracellular fatty acid composition of these cultures varied with exposure to the 
different wavelengths and the absolute fatty acid content were significantly in favor of blue light at a wave length of $470 \mathrm{~nm}$ (Das et al. 2011).

Turbidity that reduces light penetration in the culture is an important parameter determining the suitability of a culture medium for mixotrophic (and photoautotrophic) microalgae growth (Wang et al. 2010). Turbidity is specifically important in growth medium based on wastewater. Turbidity is caused by suspended matter or impurities that interfere with the clarity of the water, leading to light scattering and absorption rather than transmitted in straight lines through a water body (Wang et al. 2010). The impurities responsible for turbidity include clay, silt, suspended inorganic and organic matter, soluble colored organic compounds, plankton, and other microorganisms. Diluting wastewater in growth media affects turbidity and nutrient concentrations in the microalgae cultures and is a common solution for cultivation of microalgae (Hu et al. 2013).

Mixotrophic growth is also controlled by the interaction between organic carbon and nitrogen. Similar to heterotrophic cultures, controlling the $\mathrm{C} / \mathrm{N}$ ratio is fundamental for optimizing reactor operations (Pagnanelli et al. 2014; Silaban et al. 2014). As in all photosynthetic processes, the supply of $\mathrm{CO}_{2}$ is essential for high productivity of algae. Ideally, $\mathrm{CO}_{2}$ comes from the air supplied to the culture, but the concentration is too low to make high productivity possible. Alternatively, the $\mathrm{CO}_{2}$ is a residual gas that bubbles through the water column. As gas bubbling already requires energy, it is important that the mass transfer of $\mathrm{CO}_{2}$ is efficient (Wijffels et al. 2010).

\subsection{Advances in Bioreactor Designs}

Fermenters are adapted for most heterotrophic cultivation at the laboratory and pilot scale. Also, fermenters are suitable for commercial production of high-value products, such as food supplements and pharmaceuticals, including EPA, DHA, lutein, phycocyanin, and astaxanthin (Barclay et al. 2013; Chen and Chen 2006). Fermenters are not ideal for mixotrophic cultivation. However, fermenters made of transparent materials are used in laboratories to expose the cultures to light. Conventional fermenters and photo-bioreactors are suitable at the laboratory or pilot scale, but are very expensive at the industrial scale. Therefore, hampering production of cheap products, such as biofuels at industrial scale (Molina Grima et al. 1999; Tabernero et al. 2012). According to the economic study by Tabernero et al. (2012), the dearth of suitable bioreactors is one of the reasons preventing a biofuel facility from profitability. To make biofuel and bulk chemicals production from heterotrophic microalgae, a significant innovation is required. The conventional fermentation technologies that work very well for producing pharmaceutical compounds or high-value food supplements are just too expensive. Innovative reactor design must consider the gigantic volume of culture that is needed. At this scale, prevention of contamination from unwanted microbial species and maintenance of good mixing and oxygen supply must be resolved at the same time. With our current knowledge, we are not there. 
A good example of innovative reactor designs is the OMEGA (Offshore Membrane Enclosures for Growing Algae) system developed by NASA for cultivating microalgae from wastewater contained in floating bag photo-bioreactors deployed in marine environments. This design eliminates competition with agriculture for water, fertilizer, and land. OMEGA bioreactors use the mechanical power of waves to mix the floating photo-bioreactors and use waste $\mathrm{CO}_{2}$-rich flue gas. The reactor's wall is in contact with seawater, which is an effective way to dissipate heat (Carney et al. 2014; Wiley et al. 2013).

For evaluating bioreactors designs, it is important to compare performance of the different systems under the same operational conditions for extended periods (Wijffels et al. 2010). To facilitate quick development of the technology, research at the laboratory scale, pilot scale, and demonstration scale should run in parallel with a good interchange of information. This implies that a technology developed in the laboratory can be tested under realistic conditions in less time and research at the laboratory scale can resolve problems that would be encountered at larger scales (Wijffels et al. 2010).

\subsection{Model-Based Bioprocess Optimization}

Several mathematical modeling approaches can be used to optimize productivity of microalgae cultivation. In general many of these model-based optimization approaches belong to chemical and bioprocess engineering disciplines. They involve the practices of experimental design, model development, process design, and optimization by analyzing physical and metabolic data. Model-based process optimization is an iteractive practice where experiments in the laboratory are used to improve models and vice versa (Koide et al. 2009; Mandenius and Brundin 2008). Examples of modeling approaches for microalgae include metabolic control analysis, neural network modelling, and the response surface method (Mandenius and Brundin 2008; Nwabueze 2010; Wang et al. 2009).

Model based process optimization has been used to increase productivity of heterotrophic and mixotrophic microalgae cultivation. For instance, the Box-Wilson central composite design (CCD) is a useful computational approach that is widely used in optimizing cultivation processes. CCD was used by $\mathrm{Hu}$ et al. (2013) to develop a quadratic mathematical model for predicting the optimum dilution rate and hydraulic retention time for mass production of and nutrient removal by Chlorella sp. from swine wastewater nutrients. The development of the CCD mathematical model is based on values of the response variables, such as biomass production and nutrient removal rates in real experiments. In another example, $\mathrm{Wu}$ and Shi (2007) developed a hybrid neural network model for heterotrophic growth of Chlorella sp. and used it to optimize operational parameters of carbon source concentration and feeding rate of fed-batch cultures. After optimizing the operational parameter, culture productivity increased from 0.613 to $1.020 \mathrm{~g} \mathrm{~L}^{-1} \mathrm{~h}^{-1}$. Metabolic network analysis discovered that in the two-stage culturing mode, $\mathrm{CO}_{2}$ released 
during heterotrophic growth was fixed by the still active Rubisco enzyme that catalyzes $\mathrm{CO}_{2}$ fixation. Thus, the two-stage culturing scheme provided higher carbon consumption efficiency and lower $\mathrm{CO}_{2}$ emission, compared with heterotrophic culturing. Considering the double benefits of increased lipid yield and higher $\mathrm{CO}_{2}$ fixation, the two-stage cultivation model shows promise in the microalgae-to-biofuel field. It will be very interesting to see how the same two-stage system affects the outcomes from non-sugar carbon sources (Xiong et al. 2010). Process performance values of the above examples are presented in Table 4.

\subsection{Use of Mixed Microbial Cultures and Microalgae Consortia}

Mixed microbial cultures has been proposed as an alternative to pure cultures to reduce reactor operation costs associated with sterilization and rigorous containment under heterotrophic conditions. The use of mixed microbial cultures has been investigated mainly for microalgae growth in wastewater or in open ponds.

The highly variable composition of wastewater can limit the growth potential of specific microalgae. Therefore, it is essential to select strains capable of growing in a variety of wastewater under different climatic conditions over year-long operations. Bhatnagar et al. (2011) develop a robust microalgal consortium (strains of Chlamydomonas sp., Chlorella sp., and Scenedesmus sp.) capable of mixotrophic growth on a variety of extracts of poultry manure and carpet industry wastewater. Higher biomass and lipid production were obtained in cultures with the consortium than with single strain cultures under the same conditions. In another example, a microalgae consortium cultivated in carpet industry wastewater in raceway ponds provided higher biomass productivity and removal of nitrogen and phosphorus than single strain cultures. The consortium was developed by growing pure cultures of wastewater microalgae isolates and mixing them together in equal quantities This consortium was used for wastewater treatment and biomass production and was robust enough to withstand competition and predation in open cultivation systems (Chinnasamy et al. 2010).

An innovative approach is to use mixed cultures of microalgae and bacteria to treat wastewater (de-Bashan et al. 2002b, 2004; Hernandez et al. 2006; Covarrubias et al. 2012; Cruz et al. 2013). This approach is used to increase production of biomass and metabolites and remove nutrients from wastewaters by immobilizing microalgae strains with plant growth-promoting bacteria that also promotes and positively affect the metabolisms of the microalgae. When $C$. vulgaris was immobilized in alginate beads with Azospirillum brasilense (a microalgae growth-promoting bacteria; MGPB) and grown autotrophically on synthetic wastewater growth medium, A. brasilense mitigated environmental stress for the microalgae and removed significantly more nitrogen and phosphorus from the wastewater (deBashan et al. 2002b; de-Bashan and Bashan 2010; Hernandez et al. 2006). In another study, major cell growth occurred at $\mathrm{pH} 8$ when A. brasilense was immobilized with 
Chlorella, compared to restricted growth when Chlorella was grown alone under autotrophic conditions (de-Bashan et al. 2005). In a similar study by Perez-Garcia et al. (2010), the growth of $C$. vulgaris, under mixotrophic regimes, was higher when A. brasilense was present in the alginate beads. These studies were repeated under heterotrophic conditions and all, regardless of the cultivation regime, showed greater production of useful metabolites, such as carbohydrates (mainly starch), fatty acids, lipids, and pigments (de-Bashan et al. 2002a; Choix et al. 2012a, b, 2014; Leyva et al. 2014, 2015). In a different approach, Zhou et al. (2013) used mixed cultures of low-lipid, fast-growing mixotrophic algae (Chlorella spp. Scenedesmus obliquus, and seven other less abundant microalgae) to treat wastewater and residues from hydrothermal liquefaction of biosolids. This mix was then used to produce biomass to feed the hydrothermal liquefaction reactor to produce biofuels. The oxygen provided during algal photosynthesis reduced the energy input for aerobic breakdown of wastewater contaminants and the $\mathrm{CO}_{2}$-rich gas and residues that were produced during liquefaction were recycled back to the algal-bacterial cultivation system for reuse. Itineration of the cycle leverages the nutrient content of wastewater into high bioenergy quantities, which can be many times the original wastewater energy content (Zhou et al. 2013).

\section{Harvesting and Downstream Processes}

Economical and feasible production of biofuels from heterotrophic and mixotrophic microalgae will only be possible if biofuel production is combined with production of bulk chemicals and animal feed ingredients. It is expected that the biofuel industry and the bulk chemical industry will operate in parallel on the same biomass. Therefore, research and development of downstream processes to separate the biomass into its different molecular fractions is of utmost importance. Downstream processes involve technologies related to biomass harvesting, cell disruption, and extraction and separation technologies all working on algal biomass as raw material (Fig. 1). Industrially useful compounds such as $\omega$-3-fatty acids, carbohydrates, pigments, vitamins, and proteins should maintain their functionality after downstream processing. At the same time, scalability, low energy costs, and ease of use also need to be taken into account.

Some successful techniques commonly used in downstream processes are briefly summarized. Biomass recovery is a key step, accounting from 20 to $30 \%$ of the total operation cost of biomass production. A comprehensive description of the different harvesting methods is reviewed by Molina Grima et al. (2003). They show that for large scale production, one of the best methods is centrifugation after flocculation of the cells. Once the biomass cake is recovered, a common and widely employed method to extract oil from biomass is supercritical fluid extraction (Tabernero et al. 2012). This method recovers lipids for food supplements, such as PUFAs, or for biodiesel. In the case of biodiesel production, transesterification of extracted lipids is the major method to produce biodiesel. Alkali-catalyzed trans- 
esterification is efficient, cheap, and scalable to commercial levels (Liang 2013). An innovative approach to improve lipid extraction from biomass is to use nanomaterials, such as $\mathrm{CaO}$ and $\mathrm{MgO}$, to extract lipids without cell disruption and as biocatalyst carriers or heterogeneous catalysts in lipid transesterification to biodiesel (Zhang et al. 2013b). Another suggested approach is to break the cells by combining a low-power electromagnetic field, a process known as quantum fracturing, and adding $\mathrm{CO}_{2}$ for reducing the increasing $\mathrm{pH}$ in this process. These conditions extract the lipids with high efficiency in a single-step without chemicals, no initial dewatering, and no heavy machinery (Tabernero et al. 2012).

Whole biomass transformation methods can be employed as an alternative to avoid extraction of specific compounds from biomass. Thermochemical conversion processes convert all compounds in biomass (lipids, proteins and carbohydrates) into fuel oil or gases in a single step. Thus, this kind of transformation maximizes utilization of microalgae biomass. Thermochemical processes typically include: gasification, combustion, pyrolysis, and liquefaction. While the first two processes transform algal biomass to various gas fuels, the latter two processes produce liquid fuels (Liang 2013). Among the different thermochemical conversion processes, hydrothermal liquefaction is particularly attractive. This process converts lipids, proteins and carbohydrates into oil fuel, so the oil yield is much higher than the lipid content of the algal feedstock, as opposite to transesterification that transformed only lipids (Zhou et al. 2013).

Some limitations in downstream processes can be handled during the upstream part of the process. One approach is to avoid energy and time-consuming product extraction from biomass by developing and designing direct catalytic "sun-toproduct pathways", in which photon energy and atmospheric $\mathrm{CO}_{2}$ is directly converted into compounds that do not need further transformation after biomass production. Examples of these products would be ethanol and hydrogen. Advanced synthetic biology strategies are already being used to re-design microalgae metabolism for direct synthesis of these kinds of "ready-to-use" compounds (Robertson et al. 2011; Yu et al. 2011).

\section{Market Products and Techno-economics}

The economics of industrial production can be potentially transformed by industrial biotechnology and integrated biorefineries that produce multiple streams of valuable products (Erickson et al. 2012). To create this potential, integrated biorefineries must efficiently and simultaneously convert a broad range of industrial biomass feedstocks into affordable biofuels, energy, and a wide range of chemicals and materials. These goals are met by integrating fuel and chemical production within a single operation. High value products become an economic driver that provides higher margins of revenue to support production of low-value products (fuels), leading to a profitable biorefinery operation that also has a positive energy balance (OECD 2011). The biorefinery scheme presented in Fig. 1 illustrates this concept. 
The following streams of compounds and products are derived from refined microalgae biomass (Bassi et al. 2014; Hudek et al. 2014; Wijffels et al. 2010).

- Lipids (triacylglycerides and isoprenoids) for biodiesel;

- Lipids ( $\omega-3$ fatty acids, DHA and EPA) for nutritional supplements;

- Lipids, hydrocarbons in general, as feedstock to produce bulk chemicals and fuels;

- Proteins (soluble proteins) for nutritional supplements and personal-care products;

- Proteins (insoluble proteins) for animal feeds;

- Carbohydrates (starch, glycogen, and cellulose) as feedstock to produce bulk chemicals and fuels (i.e. bioethanol);

- Pigments (chlorophyll, $\beta$-carotene, lutein, astaxanthin) for nutrient supplements and drugs; and

- Oxygen (from photosynthesis) for general use, such as aquaculture.

- Hydrogen (from photosynthesis) used as biofuel

Nowadays, commercially successful heterotrophic microalgae production is only done for specific niche markets of high-value products, such as pigments (astaxanthin and lutein) and $\omega$-3-fatty acids (DHA and EPA) using strains of Chlorella spp., Crypthecodinium spp., Haematococcus spp., Nitzschia spp., and Schizochytrium spp. (Barclay et al. 2013; Chen and Chen 2006). As best of authors' knowledge, only Chlorella spp. and Haematococcus pluvialis strains are cultured mixotrophycally for commercial purposes (Hudek et al. 2014). Nevertheless, successful commercialization of biofuels from heterotrophic and mixotrophic microalgae relies on an integrated biorefinery. A number of biofuels and added-value chemicals can be refined from heterotrophic and mixotrophic microalgae (Bassi et al. 2014). Specifically, heterotrophic microalgae is attractive for obtaining products from cellular storage compounds, such as lipids and starch, while mixotrophic microalgae is of interest for obtaining pigments, lipids, proteins and alkanes. Heterotrophic microalgae can potentially produce large amounts of hydrocarbons and polysaccharides that can be converted into organic building blocks (ethylene, propylene, adipic acid, and furanics) for polymers and plastics (Wijffels et al. 2013). Considering the average biomass composition obtained from microalgae under different growth modes (Table 3) and the streams of compounds obtained from biorefined biomass, it is possible to anticipate the potential of each growth mode to obtain certain products, as shown in Table 6.

Biorefinery techniques should initially focus on isolating proteins and lipids because these are the largest fractions of microalgae. Other fractions, such as carbohydrates and pigments, will add significant value to the total production process when separated (Vanthoor-Koopmans et al. 2013). The main bottleneck is to separate the different fractions without damaging one or more of the product fractions. Technologies to overcome these bottlenecks need to be developed, and they should be applicable for a variety of end products of sufficient quality at large quantities. To that end, developed techniques should be gentle, inexpensive, and consume less energy. Further, locating microalgae production facilities near waste treatment 


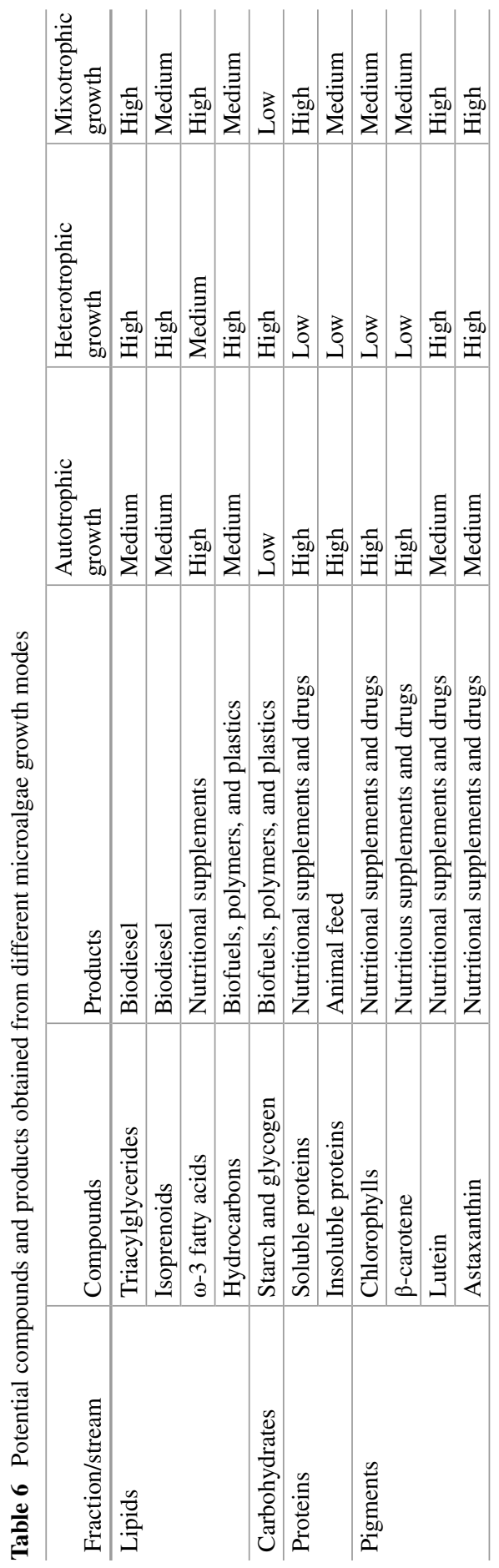


facilities, such as dairy and swine manure, food waste, or industrial organic waste could be environmentally effective and economically viable in the near future. However, heterotrophic growth is highly specific to the organic feed and microalgal species. Therefore, these waste streams have to be homogeneous and continuous if the product is biomass from a specific microalga with defined biomass composition (Kröger and Müller-Langer 2011).

Heterotrophic and mixotrophic microalgae cultivation open opportunities for the energy industry. For instance, biodiesel with good characteristics has been obtained from heterotrophic cultivation of $C$. protothecoides. For these reasons, this specific microalga is very attractive to the biodiesel production industry ( $\mathrm{Xu}$ et al. 2006). In an experimental series by Xu et al. (2006) and Miao and Wu (2006), oil extracted from heterotrophically grown microalgae was transesterified to biodiesel. The fuel was comparable to fossil diesel in density, viscosity, and heating value, and complied with the US standard for biodiesel (ASTM 6751). However, the quality of heterotrophic microalgal lipids regarding biodiesel is inferior to vegetable oil because it contains excessive free fatty acids. These fatty acids complicated the alkaline-transesterification process (Miao and Wu 2006).

Another strategy to produce biofuels from microalgae is to digest or ferment the whole biomass into methane or ethanol, respectively or process it into fuel gas (syngas, a mixture of $\mathrm{H}_{2}, \mathrm{CO}$, and $\mathrm{CO}_{2}$ ), hydrogen, or crude oil by thermo-chemical processes, such as gasification, liquefaction, and pyrolysis (Tabernero et al. 2012). Hydrogen is another compound that can be obtained by biological or thermochemical processes; however, this route is in the early stages of development (Wijffels et al. 2013). The disadvantage of using the whole biomass only for energy is that the value of more expensive compounds, such as proteins and pigments is lost (Tabernero et al. 2012).

\subsection{Techno-economic Analysis}

With current technologies, commercial success of either photo-autotrophic, heterotrophic, or mixotrophic microalgae biorefineries is unlikely. However, enormous opportunities exist to improve the current upstream and downstream technologies that can either reduce the production cost of valuable compounds or add more value. In the techno-economic analyses by Tabernero et al. (2012), a conservative and an optimized production cost of one kilogram of heterotrophic $C$. protothecoides biomass was US\$1.4 $\mathrm{kg}^{-1}$ and US\$1.19 $\mathrm{kg}^{-1}$, respectively (Table 7). These values are determined by dividing the yearly total production cost (US\$12.4 million year ${ }^{-1}$ ) by the biomass production capacity (22.7 million $\mathrm{kg} \mathrm{year}^{-1}$ ) and then adding an investment cost of US $\$ 0.93 \mathrm{~kg}^{-1}$ year $^{-1}$ for a conservative estimate or US\$0.68 kg $\mathrm{kg}^{-1}$ year $^{-1}$ for an optimized estimate. These values were estimated for a biorefinery producing biomass in 465 continuously stirred bioreactors each of $150,000 \mathrm{~L}$ and producing 10 million $\mathrm{L}$ year $^{-1}$ of biodiesel. Optimized estimates considers a higher percent of lipid recovered from biomass (from 55 to $100 \%$ ), a 
Table 7 Cost of biomass production, prices in United State dollars (USD) at a rate of Euro to dollar of 1.26 (October 2014)

\begin{tabular}{l|l|l|l}
\hline & Photo-autotrophic & Meterotrophic & Mixotrophic \\
\hline Conservative cost & US $\$ 5 \mathrm{~kg}^{-1}$ & US $\$ 1.4 \mathrm{~kg}^{-1}$ & Unknown \\
\hline Optimized cost & US\$0.5 kg & US $\$ 1.19 \mathrm{~kg}^{-1}$ & Unknown \\
\hline Reference & Wijffels et al. 2010 & Tabernero et al. 2012 & \\
\hline
\end{tabular}

an 100 ha of flat panel photobioreactors

bIn 465 continuous stirrer tank bioreactors (CSTR or fermenters) of 150,000 L, assuming oil content in biomass of $44 \%$

reduced number of bioreactors (from 465 to 257), and no oil extraction costs, based on a novel cell disruption technology using magnetism. In contrast, using a conservative approach, Wijffels et al. (2010) estimated that industrial photo-autotrophic cultivation in flat panel reactors can produce microalgal biomass at a cost of US\$11.3 $\mathrm{kg}^{-1}$ per hectare (all estimates are based on a conversion of $1.26 \mathrm{USD} /$ EUR of October 2014) The main costs of this feasibility analysis were power and labor. If the system is scaled up, labor cost is significantly reduced. A conservative cost for biomass production on $100 \mathrm{~h}$ of flat panel photo-bioreactors is about US $\$ 5 \mathrm{~kg}^{-1}$. This cost is acceptable for production of biomass for high-value compounds but unacceptable for production of biofuels. At this scale, more than $24 \%$ of the cost would be for energy, such as pumping water and sparging of air and $\mathrm{CO}_{2}$ in the system. However, production costs can be reduced by appropriate technologies. Wijffels et al. (2010) estimate an optimized cost of US $\$ 0.5 \mathrm{~kg}^{-1}$, considering the following technical aspects: free cultivation of $\mathrm{CO}_{2}$ and nutrients from wastes, reduction of energy input by $10 \%$, an increase of photosynthetic efficiency of the microalgae strain from 5 to $7 \%$, photo-bioreactors placed in a location with high levels of sunshine. The conservative and optimized costs of biomass production are summarized in Table 7. Estimates of production cost at the commercial scale of mixotrophic biomass were not found in the literature.

Once the cost of producing a kilogram of biomass is established, it is possible to analyze the total value of that kilogram of biomass after being refined into various products. Following the Wijffels et al. (2010) analysis, we assumed that microalgae biomass is refined into products for bulk chemical markets; these products and their market prices are similarly listed in Tables 8 and 9. Wijffels et al. (2010) and Xu et al. (2006) assumed different biomass compositions, depending on the growth mode of microalgae; a composition of $40 \%$ lipids, $50 \%$ proteins, and $10 \%$ carbohydrates was assumed for photo-autotrophic growth and a composition of $55 \%$ lipids, $10 \%$ protein, $15 \%$ carbohydrates, and $20 \%$ unusable compounds, such as ash and nucleic acids, was assumed for heterotrophic growth. In both analyses, $25 \%$ of the lipids fraction is used to produce bulk chemicals with a value of US\$2.5 $\mathrm{kg}^{-1}$ and the remaining $75 \%$ was for biodiesel production with a value of US $\$ 0.72 \mathrm{~kg}^{-1}$ (= US $\$ 0.63 \mathrm{~L}^{-1}$ ). The protein fraction was subdivided into a watersoluble fraction of $20 \%$ with a food value of US\$6.3 $\mathrm{kg}^{-1}$ and a water-insoluble fraction of $80 \%$ with feed value of US $\$ 0.95 \mathrm{~kg}^{-1}$. Finally, $100 \%$ of the carbohydrate 
Table 8 Value of biomass and profit obtained from photo-autotrophic microalgae

\begin{tabular}{|c|c|c|c|}
\hline Product & $\begin{array}{l}\text { Percentage of biomass } \\
\text { dry weight composition } \\
(\%)\end{array}$ & $\begin{array}{l}\text { Price per } \mathrm{kg} \\
\text { of product } \\
\left(\mathrm{US} \$ \mathrm{~kg}^{-1}\right)\end{array}$ & $\begin{array}{l}\text { Product value in } \\
1000 \mathrm{~kg} \text { of biomass } \\
\text { (US\$) }\end{array}$ \\
\hline Biofuels from lipids & 30 & 0.72 & $\$ 214$ \\
\hline Bulk chemicals from lipids & 10 & 2.52 & $\$ 252$ \\
\hline $\begin{array}{l}\text { Nutritious supplements from } \\
\text { soluble protein }\end{array}$ & 10 & 6.30 & $\$ 630$ \\
\hline Feeds from insoluble protein & 40 & 0.95 & $\$ 378$ \\
\hline $\begin{array}{l}\text { Bulk chemicals from } \\
\text { carbohydrates }\end{array}$ & 10 & 1.26 & $\$ 126$ \\
\hline Oxygen & - & 0.20 & $\$ 322$ \\
\hline $\mathrm{N}$ removal & - & 2.58 & $\$ 170$ \\
\hline Total & 100 & & $\$ 2,099$ \\
\hline $\begin{array}{l}\text { Conservative production } \\
\operatorname{cost}^{\mathrm{b}}\end{array}$ & & & $\$ 5,000$ \\
\hline Optimized production $\cos ^{\mathrm{b}}$ & & & $\$ 500$ \\
\hline NET PROFIT Conservative ${ }^{\mathrm{b}}$ & & & $-\$ 2,901$ \\
\hline NET PROFIT Optimized $^{c}$ & & & $+\$ 1,599$ \\
\hline
\end{tabular}

aProduct value in a $1000 \mathrm{~kg}$ of biomass $=\%$ of composition (in decimals) multiplied by the price of $\mathrm{kg}$ of product multiplied by a thousand

${ }^{b}$ From Table 7 multiplied by a thousand

${ }^{\mathrm{C}} \mathrm{Net}$ profit $=$ Total value of a $1000 \mathrm{~kg}$ of biomass minus production cost

fraction has a market value of US\$1.26 kg-1 and consist of cellular storage products, such as starch, glucans, and glycerol that can be used as chemical building blocks or production of energy. Besides these main products, there are additional by-products or valuable environmental services, such as reducing nutrients in waste streams and production of oxygen. In wastewater treatment, removal of nitrogen compounds by nitrification and denitrification is an expensive process. The cost of nitrogen removal is US\$2.52 $\mathrm{kg}^{-1}$. Microalgae contain $70 \mathrm{~kg}$ of nitrogen per $1000 \mathrm{~kg}$ of microalgae. If microalgae production is combined with wastewater treatment, we can save US $\$ 176$ for nitrification and denitrification per ton of microalgae produced. Photoautotrophic algae produces oxygen. Per ton of microalgae, $1600 \mathrm{~kg}$ of oxygen-rich gas is produced with an approximate value of US\$0.2 $\mathrm{kg}^{-1}$, resulting in US\$ 322 ton $^{-1}$. The percentage of biomass composition and the market price of those components or fractions are listed in Tables 8 and 9.

Analyses presented in Tables 8 and 9 show that, if microalgal biorefining is used, the total value of the biomass produced using photo-autotrophic or heterotrophic cultivation of microalgae (US\$2,099 and US $\$ 1,249$ per $1000 \mathrm{~kg}$, respectively) is higher than the optimized costs for microalgae production, but lower than the conservative costs. Considering that conservative costs were estimated with current technology and operating standards, a general analysis shows that heterotrophic cultivation is currently too expensive to be implemented in a sustainable and commercially feasible microalgae biorefinery. This high cost results mainly from the many bioreactors required by the plant and the yields of oil (Tabernero et al. 2012). 
Table 9 Value of biomass and profit obtained from heterotrophic microalgae

\begin{tabular}{|c|c|c|c|}
\hline Product & $\begin{array}{l}\text { Percentage of biomass } \\
\text { dry weight composition } \\
(\%)\end{array}$ & $\begin{array}{l}\text { Price per kg of } \\
\text { product (US\$/ } \\
\mathrm{kg})\end{array}$ & $\begin{array}{l}\text { Product value in } \\
1000 \mathrm{~kg} \text { of biomass } \\
\text { (US\$) }\end{array}$ \\
\hline Biofuels from lipids & 41 & 0.72 & $\$ 296$ \\
\hline Bulk chemicals from lipids & 14 & 2.52 & $\$ 348$ \\
\hline $\begin{array}{l}\text { Nutritious supplements } \\
\text { from soluble protein }\end{array}$ & 3 & 6.30 & $\$ 162$ \\
\hline $\begin{array}{l}\text { Feeds from insoluble } \\
\text { protein }\end{array}$ & 8 & 0.95 & $\$ 73$ \\
\hline $\begin{array}{l}\text { Bulk chemicals from } \\
\text { carbohydrates }\end{array}$ & 15 & 1.26 & $\$ 194$ \\
\hline Oxygen & & & $\$ 0$ \\
\hline $\mathrm{N}$ removal & & & $\$ 176$ \\
\hline Total & 100 & & $\$ 1,249$ \\
\hline $\begin{array}{l}\text { Conservative production } \\
\text { cost }^{\text {b }}\end{array}$ & & & $\$ 1,478$ \\
\hline Optimized production $\operatorname{cost}^{\mathrm{b}}$ & & & $\$ 1,196$ \\
\hline $\begin{array}{l}\text { NET PROFIT } \\
\text { Conservative }^{\text {b }}\end{array}$ & & & $-\$ 229$ \\
\hline NET PROFIT Optimized ${ }^{\mathrm{c}}$ & & & $+\$ 54$ \\
\hline
\end{tabular}

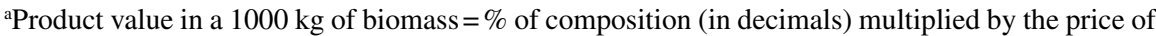
$\mathrm{kg}$ of product multiplied by a thousand

${ }^{b}$ From Table 7 multiplied by a thousand

${ }^{\mathrm{c}} \mathrm{Net}$ profit $=$ Total value of a $1000 \mathrm{~kg}$ of biomass minus production cost

Further, the analysis shows that heterotrophic biomass has a lower value than photoautotrophic biomass because it contains less soluble proteins, which is the biomass fraction with highest market value.

This analysis also illustrates the necessity to refine microalgae biomass into various products. If we assume an optimized heterotrophic biomass production cost of US $\$ 1.18 \mathrm{~kg}^{-1}$, the algae contains $55 \%$ lipids and that is possible to transform $100 \%$ of these lipids into biodiesel, the cost to produce $1 \mathrm{~kg}$ of biodiesel from heterotrophic microalgae would be US\$1.81 $\mathrm{kg}^{-1}$. This is more than double the US\$0.715 $\mathrm{kg}^{-1}$ the average market value of petroleum diesel in October 2014 in the United States (source: U.S. Energy Information Administration. $\sim \mathrm{US} \$ 0.715 \mathrm{~kg}^{-1}=\mathrm{US} \$ 0.63 \mathrm{~L}^{-1}$, considering a biodiesel density of $0.88 \mathrm{~kg} \mathrm{~L}^{-1}$ ). For this reason, refining microalgal biomass into different products is essential to increase the total value of the biomass. If microalgal biomass is refined, its total value approaches $U S \$ 1.25 \mathrm{~kg}^{-1}$ (Table 9), slightly higher than the total cost for microalgae production (US\$1.18 $\mathrm{kg}^{-1}$ ). These results reiterate that the economics of microalgal biofuel production would not be competitive with traditional fossil fuels if a large scale facility were to be built today (Davis et al. 2011). The crucial question is whether microalgal biomass can be produced at a cost below US\$1.18 or US\$0.5 $\mathrm{kg}^{-1}$, using heterotrophic or photo-autotrophic cultivation (Wijffels et al. 2010). 
Our analysis, combined with studies by Brentner et al. (2011), Davis et al. (2011), Kröger and Müller-Langer (2011), Tabernero et al. (2012), and Wijffels et al. (2010) demonstrate that economically feasible co-production of heterotrophic microalgae biofuels and other chemicals is possible if the current technology develops and significantly reduces production and refining cost. In every analysis, the two highest costs are attributed to bioreactor set up and harvesting costs. Therefore, new technological developments should focus mainly on these aspects of the bioprocess. Sections 3, 4, 5, and 6 of this chapter discuss in detail technological advances currently under development. A sensitivity analysis of a mathematical model of microalgae biomass production process pointed out that the content of valuable compounds in biomass is the most sensitive factor affecting costs of bioreactors operations. This means that, by increasing the content of valuable compounds in biomass, it is possible to effectively reduce operation costs. This emphasizes the importance of strain selection and development of the appropriate processes of cultivation and harvesting (Davis et al. 2011). Additionally, the performance and economic feasibility of a microalgal biorefinery will vary greatly with location, depending on the physical and technological environment in which it is located and the cost of labor at the site. For mixotrophic microalgae, parameters, such as ambient temperature and solar intensity can clearly affect the potential growth rate of microalgae and costs of bioreactors (Brentner et al. 2011).

Mixotrophic growth offers several features, which should be examined more closely. An increase in growth rate and production of valuable compounds by adding an organic substrate to a photo-autotrophic culture is possible. Therefore, the use of a mixotrophic regime is a logical step to stabilize and enhance the production process. High-production refineries will be built in the coming years and mixotrophy is a good strategy to minimize their default risk (Kröger and Müller-Langer 2011). It remains to be investigated if, and for which species, the addition of organic substrates provide a more robust and efficient operation. The possibilities of mixotrophy should also be considered when designing a microalgae production facility, since they may have an impact on the production rate and reliability and, therefore, economics (Kröger and Müller-Langer 2011).

\section{Final Remarks}

Heterotrophic and mixotrophic cultivation of microalgae offers an alternative to photo-autotrophic cultivation to improve the economic feasibility of microalgae biorefined products. Although microalgae are not yet cultivated at large scales for bulk applications, there are opportunities to develop this process in a sustainable way in the foreseeable future. With the current technologies in place, it remains unlikely, however, that the process will be developed for biodiesel as the sole endproduct from microalgae biomass because no biofuel production from microalgae comes close to current market prices of fossil fuels. Consequently, to develop a more sustainable and economically feasible process, all biomass components, such as proteins, lipids, and carbohydrates should be used to obtain compounds with high 
market value. Therefore, in biorefining of microalgae, the selective separation and use of the functional biomass components is of utmost importance. If, in addition, production of microalgae biomass is done on wastewater or industrial wastes as the primary substrate, without special production of growth medium ingredients, and the cultivation is done on a large scale at low costs, production of bulk chemicals and fuels from microalgae may become economically feasible (Wijffels et al. 2010).

For producing biofuels and bulk chemicals from lipids and carbohydrates, cultivating microalgae under heterotrophic and mixotrophic regimes offers great promise because high production of biomass and lipids or carbohydrates (but not proteins) can be expected, specially using fed-batch cultivation modes. Livestock manures, molasses, food wastes, and whey from the dairy industry were shown to be promising organic substrates for heterotrophic and mixotrophic cultivation. Further research on these substrates should analyze, in detail, yields, volumetric productivity, and product concentration values of different microalgal strains on these potential substrates.

To make heterotrophic and mixotrophic cultivation commercially viable, research should focus on:

- Lowering the cost of carbon substrates;

- Bio-prospecting or metabolically engineering microalgae strains able to assimilate multiple substrates, resist changing environmental conditions, and technical condition prevailing during industrial production and have fast growth;

- Determining the potential of consortia of microalgae or microalgae with bacteria in natural co-cultivation (microorganisms from similar habitat) or in synthetic biology assemblage (microorganisms not from the same origin);

- Design suitable bioreactors at industrial scales that are made of cheap materials adjusted to the needs of microalgae cultivation; and

- Optimizing downstream processes of transforming microalgal biomass to various produces, including fuels.

With intensive research and development currently underway in these fields, it is reasonable to anticipate that large-scale biofuel production combined with an industry of high-value products from heterotrophic and mixotrophic microalgae, probably fueled by waste products from other industries, will be a reality in the foreseeable future.

Acknowledgement We thank Ira Fogel of CIBNOR Mexico for editing the manuscript. Time for writing was provided by The Bashan Foundation, USA.

\section{Acronyms List}

$\begin{array}{ll}\mu^{\max } & \text { Maximum growth rate } \\ \text { BOD } & \text { Biological oxygen demand } \\ \text { C/N } & \text { Carbon to nitrogen ratio of growth media } \\ \text { CCD } & \text { Central composite design }\end{array}$




$\begin{array}{ll}\text { COD } & \text { Chemical oxygen demand } \\ \text { DHA } & \text { Docosahexaenoic acid } \\ \text { EC } & \text { Enzyme commission numer } \\ \text { EM pathway } & \text { Embden-Meyerhof pathway } \\ \text { EPA } & \text { Eicosapentaenoic acid } \\ \text { FBA } & \text { Flux balance analysis } \\ \text { HRT } & \text { Hydraulic retention time } \\ \text { LEDs } & \text { Light-emitting diodes } \\ \text { mRNA } & \text { Messenger RNA } \\ \text { OMEGA } & \text { Offshore Membrane Enclosures for Growing Algae } \\ \text { PCR } & \text { Polymerase chain reaction } \\ \text { PP pathway } & \text { Pentose phosphate pathway } \\ \text { PUFAs } & \text { Polyunsaturated fatty acids } \\ \text { SMN } & \text { Stoichiometric metabolic network } \\ \text { STR } & \text { Stirred tank bioreactor } \\ \text { TCA cycle } & \text { Tricarboxylic acid cycle } \\ \text { VSS } & \text { Dissolved volatile solids }\end{array}$

\section{References}

Andrade MR, Costa JAV (2007) Mixotrophic cultivation of microalga Spirulina platensis using molasses as organic substrate. Aquaculture 264:130-134

Aubert S, Gout E, Bligny R, Douce R (1994) Multiple effects of glycerol on plant cell metabolism. Phosphorus-31 nuclear magnetic resonance studies. J Biol Chem 269:21420-21427

Barclay W, Apt K (2013) Strategies for bioprospecting microalgae for potential commercial applications. In: Richmond A, Hu Q (eds) Handbook of microalgal culture: applied phycology and biotechnology, 2nd edn. Wiley-Blackwell, Chichester, pp 69-79

Barclay W, Apt K, Dong XD (2013) Commercial production of microalgae via fermentation. In: Richmond A, Hu Q (eds) Handbook of microalgal culture: applied phycology and biotechnology, 2nd edn. Wiley-Blackwell, Chichester, pp 134-145

Bassi A, Saxena P, Aguirre AM (2014) Mixotrophic algae cultivation for energy production and other applications. In: Bajpai R, Prokop A, Zappi M (eds) Algal biorefineries, Vol. 1. Cultivation of cells and products. Springer, New York, pp 177-202

Behrens PW (2005) Photobioreactor and fermentors: the light and the dark sides of the growing algae. In: Andersen RA (ed) Algal culturing techniques. Elsevier Academic Press, New York, pp 189-203

Bhatnagar A, Chinnasamy S, Singh M, Das KC (2011) Renewable biomass production by mixotrophic algae in the presence of various carbon sources and wastewaters. Appl Energy 88:3425-3431

Blifernez-Klassen O, Klassen V, Doebbe A, Kersting K, Grimm P, Wobbe L, Kruse O (2012) Cellulose degradation and assimilation by the unicellular phototrophic eukaryote Chlamydomonas reinhardtii. Nat Commun 3:1214

Bollman RC, Robinson GGC (1977) The kinetics of organic acid uptake by three Chlorophyta in axenic culture. J Phycol 13:1-5

Borowitzka MA (1999) Commercial production of microalgae: ponds, tanks, tubes and fermenters. J Biotechnol 70:313-321

Boyle NR, Morgan JA (2009) Flux balance analysis of primary metabolism in Chlamydomonas reinhardtii. BMC Syst Biol 3:4. doi:10.1186/1752-0509-3-4 
Brányiková I, Marsálková B, Doucha J, Brányik T, Bisová K, Zachleder V, Vitova M (2011) Microalgae - novel highly efficient starch producers. Biotechnol Bioeng 108:766-776

Brennan L, Owende P (2010) Biofuels from microalgae - a review of technologies for production, processing, and extractions of biofuels and co-products. Renew Sustain Energy Rev 14:557-577

Brentner LB, Eckelman MJ, Zimmerman JB (2011) Combinatorial life cycle assessment to inform process design of industrial production of algal biodiesel. Environ Sci Technol 45:7060-7067

Carney LT, Reinsch SS, Lane PD, Solberg OD, Jansen LS, Williams KP, Trent JD, Lane TW (2014) Microbiome analysis of a microalgal mass culture growing in municipal wastewater in a prototype OMEGA photobioreactor. Algal Res 4:52-61

Cha TS, Chen JW, Goh EG, Aziz A, Loh SH (2011) Differential regulation of fatty acid biosynthesis in two Chlorella species in response to nitrate treatments and the potential of binary blending microalgae oils for biodiesel application. Bioresour Technol 102:10633-10640

Chang RL, Ghamsari L, Manichaikul A, Hom EFY, Balaji S, Fu W, Shen Y, Hao T, Palsson BØ, Salehi-Ashtiani K, Papin JA (2011) Metabolic network reconstruction of Chlamydomonas offers insight into light-driven algal metabolism. Mol Syst Biol 7:518. doi:10.1038/msb.2011.52

Charles DF, Knowles D, Davis RS (2002) Protocols for the analysis of algal samples collected as part of the U.S. Geological Survey National Water-Quality Assessment Program. Report No. 02-06, Philadelphia

Cheirsilp B, Torpee S (2012) Enhanced growth and lipid production of microalgae under mixotrophic culture condition: effect of light intensity, glucose concentration and fed-batch cultivation. Bioresour Technol 110:510-516

Chen F (1996) High cell density culture of microalgae in heterotrophic growth. Trends Biotechnol 14:421-426

Chen GQ, Chen F (2006) Growing phototrophic cells without light. Biotechnol Lett 28:607-616

Chen F, Zhang Y, Guo S (1996) Growth and phycocyanin formation of Spirulina platensis in photoheterotrophic culture. Biotechnol Lett 18:603-608

Chen CY, Yeh KL, Aisyah R, Lee DJ, Chang JS (2011) Cultivation, photobioreactor design and harvesting of microalgae for biodiesel production: a critical review. Bioresour Technol 102:71-81

Cheng Y, Lu Y, Gao C, Wu Q (2009a) Alga-based biodiesel production and optimization using sugar cane as the feedstock. Energy Fuel 23:4166-4173

Cheng Y, Zhou W, Gao C, Lan K, Gao Y, Wu Q (2009b) Biodiesel production from Jerusalem artichoke (Helianthus Tuberosus L.) tuber by heterotrophic microalgae Chlorella protothecoides. J Chem Technol Biotechnol 84:777-781

Chi Z, Pyle D, Wen Z, Frear C, Chen S (2007) A laboratory study of producing docosahexaenoic acid from biodiesel-waste glycerol by microalgal fermentation. Process Biochem 42:1537-1545

Chinnasamy S, Bhatnagar A, Hunt RW, Das KC (2010) Microalgae cultivation in a wastewater dominated by carpet mill effluents for biofuel applications. Bioresour Technol 101:3097-3105

Choix FJ, de-Bashan LE, Bashan Y (2012a) Enhanced accumulation of starch and total carbohydrates in alginate-immobilized Chlorella spp. induced by Azospirillum brasilense: II. Heterotrophic conditions. Enzyme Microb Technol 51:300-309

Choix FJ, de-Bashan LE, Bashan Y (2012b) Enhanced accumulation of starch and total carbohydrates in alginate-immobilized Chlorella spp. induced by Azospirillum brasilense: I. Autotrophic conditions. Enzyme Microb Technol 51:294-299

Choix FJ, Bashan Y, Mendoza A, de-Bashan LE (2014) Enhanced activity of ADP glucose pyrophosphorylase and formation of starch induced by Azospirillum brasilense in Chlorella vulgaris. J Biotechnol 177:22-34

Chojnacka K, Marquez-Rocha F (2004) Kinetic and stoichiometric relationships of the energy and carbon metabolism in the culture of microalgae. Biotechnology 4:21-34

Chojnacka K, Noworyta A (2004) Evaluation of Spirulina sp. growth in photoautotrophic, heterotrophic and mixotrophic cultures. Enzyme Microb Technol 34:461-465 
Christenson L, Sims R (2011) Production and harvesting of microalgae for wastewater treatment, biofuels, and bioproducts. Biotechnol Adv 29:686-702

Covarrubias SA, de-Bashan LE, Moreno M, Bashan Y (2012) Alginate beads provide a beneficial physical barrier against native microorganisms in wastewater treated with immobilized bacteria and microalgae. Appl Microbiol Biotechnol 93:2669-2680

Cruz I, Bashan Y, Hernandez-Carmona G, de-Bashan LE (2013) Biological deterioration of alginate beads containing immobilized microalgae and bacteria during tertiary wastewater treatment. Appl Microbiol Biotechnol 97:9847-9858

Das P, Lei W, Aziz SS, Obbard JP (2011) Enhanced algae growth in both phototrophic and mixotrophic culture under blue light. Bioresour Technol 102:3883-3887

Davis R, Aden A, Pienkos PT (2011) Techno-economic analysis of autotrophic microalgae for fuel production. Appl Energy 88:3524-3531

Day JD, Edwards AP, Rodgers GA (1991) Development of an industrial-scale process for the heterotrophic production of a micro-algal mollusc feed. Bioresour Technol 38:245-249

De Swaaf ME, Sijtsma L, Pronk JT (2003a) High-cell-density fed-batch cultivation of the docosahexaenoic acid producing marine alga Crypthecodinium cohnii. Biotechnol Bioeng 81:666-672

De Swaaf ME, Pronk JT, Sijtsma L (2003b) Fed-batch cultivation of the docosahexaenoic-acidproducing marine alga Crypthecodinium cohnii on ethanol. Appl Microbiol Biotechnol 61:40-43

de-Bashan LE, Bashan Y (2010) Immobilized microalgae for removing pollutants: review of practical aspects. Bioresour Technol 101:1611-1627

de-Bashan LE, Bashan Y, Moreno M, Lebsky VK, Bustillos JJ (2002a) Increased pigment and lipid content, lipid variety, and cell and population size of the microalgae Chlorella spp. when coimmobilized in alginate beads with the microalgae-growth-promoting bacterium Azospirillum brasilense. Can J Microbiol 48:514-521

de-Bashan LE, Moreno M, Hernandez J-P, Bashan Y (2002b) Removal of ammonium and phosphorus ions from synthetic wastewater by the microalgae Chlorella vulgaris coimmobilized in alginate beads with the microalgae growth-promoting bacterium Azospirillum brasilense. Water Res 36:2941-2948

de-Bashan LE, Hernandez J-P, Morey T, Bashan Y (2004) Microalgae growth-promoting bacteria as "helpers" for microalgae: a novel approach for removing ammonium and phosphorus from municipal wastewater. Water Res 38:466-474

de-Bashan LE, Antoun H, Bashan Y (2005) Cultivation factors and population size control the uptake of nitrogen by the microalgae Chlorella vulgaris when interacting with the microalgae growth-promoting bacterium Azospirillum brasilense. FEMS Microbiol Ecol 54:197-203

Dorian PM (1995) Bioprocess engineering principles. Academic, London

Dragone G, Fernandes BD, Abreu AP, Vicente AA, Teixeira JA (2011) Nutrient limitation as a strategy for increasing starch accumulation in microalgae. Appl Energy 88:3331-3335

Droop MR (1974) Heterotrophy of carbon. In: Stewart WDP (ed) Algal physiology and biochemistry. Blackwell Scientific, Oxford, pp 530-559

Ducat DC, Avelar-Rivas JA, Way JC, Silvera PA (2012) Rerouting carbon flux to enhance photosynthetic productivity. Appl Environ Microbiol 78:2660-2668

Erickson B, Nelson WP (2012) Perspective on opportunities in industrial biotechnology in renewable chemicals. Biotechnol J 7:176-185

Espinosa-Gonzalez I, Parashar A, Bressler DC (2014) Heterotrophic growth and lipid accumulation of Chlorella protothecoides in whey permeate, a dairy by-product stream, for biofuel production. Bioresour Technol 155:170-176

Gao C, Zhai Y, Ding Y, Wu Q (2010) Application of sweet sorghum for biodiesel production by heterotrophic microalga Chlorella protothecoides. Appl Energy 87:756-761

Gao Z, Zhao H, Li Z, Tan X, Lu X (2012) Photosynthetic production of ethanol from carbon dioxide in genetically engineered cyanobacteria. Energy Environ Sci 5:9857-9865

García-Ferris C, De Los RA, Ascaso C, Moreno J (1996) Correlated biochemical and ultrastructural changes in nitrogen-starved Euglena gracilis. J Phycol 32:953-963 
Geider RJ, Osborne BA (1989) Respiration and microalgal growth: a review of the quantitative relationship between dark respiration and growth. New Phytol 112:327-341

Girard J, Roy M, Hafsa MB, Gagnon J, Faucheux N, Heitz M, Tremblay R, Deschênes J (2014) Mixotrophic cultivation of green microalgae Scenedesmus obliquus on cheese whey permeate for biodiesel production. Algal Res 5:241-248

Göksan T, Ak I, Gökpinar S (2010) An alternative approach to the traditional mixotrophic cultures of Haematococcus pluvialis Flotow (Chlorophyceae). J Microbiol Biotechnol 20:1276-1282

Graverholt OS, Eriksen NT (2007) Heterotrophic high-cell-density fed-batch and continuous-flow cultures of Galdieria sulphuraria and production of phycocyanin. Appl Microbiol Biotechnol 77:69-75

Griffiths DJ, Thresher CL, Street HE (1960) The heterotrophic nutrition of Chlorella vulgaris (brannon no. 1 strain): with two figures in the text. Ann Bot 24:1-11

Hawkins RL (1999) Utilization of xylose for growth by the eukaryotic alga, Chlorella. Curr Microbiol 38:360-363

Heredia-Arroyo T, Wei W, Hu B (2010) Oil accumulation via heterotrophic/mixotrophic chlorella protothecoides. Appl Biochem Biotechnol 162:1978-1995

Hernandez JP, de-Bashan LE, Bashan Y (2006) Starvation enhances phosphorus removal from wastewater by the microalgae Chlorella spp. co-immobilized with Azospirillum brasilense. Enzyme Microb Technol 38:190-198

Hillebrand H, Sommer U (1999) The nutrient stoichiometry of benthic microalgal growth: redfield proportions are optimal. Limnol Oceanogr 44:440-446

Hirano A, Ueda R, Hirayama S, Ogushi Y (1997) $\mathrm{CO}_{2}$ fixation and ethanol production with microalgal photosynthesis and intracellular anaerobic fermentation. Energy 22:137-142

Hong SJ, Lee CG (2007) Evaluation of central metabolism based on a genomic database of Synechocystis PCC6803. Biotechnol Bioprocess Eng 12:165-173

Hsieh CH, Wu WT (2009) Cultivation of microalgae for oil production with a cultivation strategy of urea limitation. Bioresour Technol 100:3921-3926

Hu B, Min M, Zhou W, Li Y, Mohr M, Cheng Y, Lei H, Liu Y, Lin X, Chen P, Ruan R (2012) Influence of exogenous $\mathrm{CO}_{2}$ on biomass and lipid accumulation of microalgae Auxenochlorella protothecoides cultivated in concentrated municipal wastewater. Appl Biochem Biotechnol 166:1661-1673

Hu B, Zhou W, Min M, Du Z, Chen P, Ma X, Liu Y, Lei H, Shi J, Ruan R (2013) Development of an effective acidogenically digested swine manure-based algal system for improved wastewater treatment and biofuel and feed production. Appl Energy 107:255-263

Hudek KL, Davis LC, Ibbini J, Erickson LE (2014) Commercial products from algae. In: Bajpai R, Prokop A, Zappi M (eds) Algal biorefineries, Vol. 1. Cultivation of cells and products. Springer, New York, pp 275-295

Illman AM, Scragg AH, Shales SW (2000) Increase in Chlorella strains calorific values when grown in low nitrogen medium. Enzyme Microb Technol 27:631-635

John RP, Anisha GS, Nampoothiri KM, Pandey A (2011) Micro and macroalgal biomass: a renewable source for bioethanol. Bioresour Technol 102:186-193

Kanehisa M, Goto S (2000) KEGG: Kyoto encyclopedia of genes and genomes. Nucleic Acids Res 28:27-30. Database website: http://www.genome.jp/kegg/

Kang R, Wang J, Shi D, Cong W, Cai Z, Ouyang F (2004) Interactions between organic and inorganic carbon sources during mixotrophic cultivation of Synechococcus sp. Biotechnol Lett 26:1429-1432

Khozin-Goldberg I, Cohen Z (2006) Effect of phosphate starvation on lipid and fatty acid composition of the eustigmatophyte Monodus subterraneus. Phytochemistry 67:696-701

Khozin-Goldberg I, Cohen Z (2011) Unraveling algal lipid metabolism: recent advances in gene identification. Biochimie 93:91-100

Koide T, Lee Pang W, Baliga NS (2009) The role of predictive modelling in rationally reengineering biological systems. Nat Rev Microbiol 7:297-305

Kröger M, Müller-Langer F (2011) Impact of heterotrophic and mixotrophic growth of microalgae on the production of future biofuels. Biofuels 2:145-151 
Lebsky VK, Gonzalez-Bashan LE, Bashan Y (2001) Ultrastructure of interaction in alginate beads between the microalga Chlorella vulgaris with its natural associative bacterium Phyllobacterium myrsinacearum and with the plant growth-promoting bacterium Azospirillum brasilense. Can J Microbiol 47:1-8

Lee SK, Chou H, Ham TS, Lee TS, Keasling JD (2008) Metabolic engineering of microorganisms for biofuels production: from bugs to synthetic biology to fuels. Curr Opin Biotechnol 19:556-563

León-Bañares R, González-Ballester D, Galván A, Fernández E (2004) Transgenic microalgae as green cell-factories. Trends Biotechnol 22:45-52

Leyva LA, Bashan Y, Mendoza A, de-Bashan LE (2014) Accumulation of fatty acids in Chlorella vulgaris under heterotrophic conditions in relation to activity of acetyl-CoA carboxylase, temperature, and co-immobilization with Azospirillum brasilense. Naturwissenschaften 101:819-830

Leyva LA, Bashan Y, de-Bashan LE (2015) Activity of acetyl-CoA carboxylase is not directly linked to accumulation of lipids when Chlorella vulgaris is co-immobilized with Azospirillum brasilense in alginate under autotrophic and heterotrophic conditions. Ann Microbiol 65:339-349.

Li X, Xu H, Wu Q (2007) Large-scale biodiesel production from microalga Chlorella protothecoides through heterotrophic cultivation in bioreactors. Biotechnol Bioeng 98:764-771

Li Y, Horsman M, Wang B, Wu N, Lan CQ (2008) Effects of nitrogen sources on cell growth and lipid accumulation of green alga Neochloris oleoabundans. Appl Microbiol Biotechnol 81:629-636

Li Y, Zhou W, Hu B, Min M, Chen P, Ruan RR (2011) Integration of algae cultivation as biodiesel production feedstock with municipal wastewater treatment: strains screening and significance evaluation of environmental factors. Bioresour Technol 102:10861-10867

Li Y, Zhou W, Hu B, Min M, Chen P, Ruan RR (2012) Effect of light intensity on algal biomass accumulation and biodiesel production for mixotrophic strains Chlorella kessleri and Chlorella protothecoides cultivated in highly concentrated municipal wastewater. Biotechnol Bioeng 109:2222-2229

Liang Y (2013) Producing liquid transportation fuels from heterotrophic microalgae. Appl Energy 104:860-868

Liang Y, Sarkany N, Cui Y (2009) Biomass and lipid productivities of Chlorella vulgaris under autotrophic, heterotrophic and mixotrophic growth conditions. Biotechnol Lett 31:1043-1049

Lloyd D (1974) Dark respiration. In: Stewart WDP (ed) Algal physiology and biochemistry. Blackwell Scientific Publications, Oxford, pp 505-529

Lu Y, Zhai Y, Liu M, Wu Q (2010) Biodiesel production from algal oil using cassava (Manihot esculenta Crantz) as feedstock. J Appl Phycol 22:573-578

Mandenius CF, Brundin A (2008) Bioprocess optimization using design-of-experiments methodology. Biotechnol Prog 24:1191-1203

Marquez FJ, Sasaki K, Kakizono T, Nishio N, Nagai S (1993) Growth characteristics of Spirulina platensis in mixotrophic and heterotrophic conditions. J Ferment Bioeng 76:408-410

Merzlyak MN, Chivkunova OB, Gorelova OA, Reshetnikova IV, Solovchenko AE, KhozinGoldberg I, Cohen Z (2007) Effect of nitrogen starvation on optical properties, pigments and arachidonic acid content of the unicellular green alga Parietochloris incisa (trebouxiophyceae) chlorophyta. J Phycol 43:833-843

Miao X, Wu Q (2006) Biodiesel production from heterotrophic microalgal oil. Bioresour Technol 97:841-846

Molina Grima E, Fernández FGA, García Camacho F, Chisti Y (1999) Photobioreactors: light regime, mass transfer, and scaleup. J Biotechnol 70:231-247

Molina Grima E, Belarbi E, Fernández FGA, Robles Medina A, Chisti Y (2003) Recovery of microalgal biomass and metabolites: process options and economics. Biotechnol Adv 20:491-515

Neilson AH, Lewin RA (1974) The uptake and utilization of organic carbon by algae: an essay in comparative biochemistry. Phycologia 13:227-264 
Nwabueze TU (2010) Basic steps in adapting response surface methodology as mathematical modelling for bioprocess optimisation in the food systems. Int J Food Sci Technol 45:1768-1776

Oberhardt MA, Chavali AK, Papin JA (2009) Flux balance analysis: interrogating genome-scale metabolic networks. Method Mol Biol 500:61-80

OECD (2011) Future prospects for industrial biotechnology. OECD Publishing, Paris, http://dx. doi.org/10.1787/9789264126633-en

Ogbonna JC, Yoshizawa H, Tanaka H (2000) Treatment of high strength organic wastewater by a mixed culture of photosynthetic microorganisms. J Appl Phycol 12:277-284

Ogbonna JC, Ichige E, Tanaka H (2002) Interactions between photoautotrophic and heterotrophic metabolism in photoheterotrophic cultures of Euglena gracilis. Appl Microbiol Biotechnol 58:532-538

Orth JD, Thiele I, Palsson BO (2010) What is flux balance analysis? Nat Biotechnol 28:245-248

Pagnanelli F, Altimari P, Trabucco F, Toro L (2014) Mixotrophic growth of Chlorella vulgaris and Nannochloropsis oculata: interaction between glucose and nitrate. J Chem Technol Biotechnol 89:652-661

Pal D, Khozin-Goldberg I, Cohen Z, Boussiba S (2011) The effect of light, salinity, and nitrogen availability on lipid production by Nannochloropsis sp. Appl Microbiol Biotechnol 90:1429-1441

Park KC, Whitney C, McNichol JC, Dickinson KE, MacQuarrie S, Skrupski BP, Zou J, Wilson KE, O'Leary SJB, McGinn PJ (2012) Mixotrophic and photoautotrophic cultivation of 14 microalgae isolates from Saskatchewan, Canada: potential applications for wastewater remediation for biofuel production. J Appl Phycol 24:339-348

Perez-Garcia O, de-Bashan LE, Hernandez J, Bashan Y (2010) Efficiency of growth and nutrient uptake from wastewater by heterotrophic, autotrophic, and mixotrophic cultivation of Chlorella vulgaris immobilized with Azospirillum brasilense. J Phycol 46:800-812

Perez-Garcia O, Bashan Y, Puente ME (2011a) Organic carbon supplementation of sterilized municipal wastewater is essential for heterotrophic growth and removing ammonium by the microalga Chlorella vulgaris. J Phycol 47:190-199

Perez-Garcia O, Escalante FME, de-Bashan LE, Bashan Y (2011b) Heterotrophic cultures of microalgae: metabolism and potential products. Water Res 45:11-36

Perez-Garcia O, Villas-Boas SG, Swift S, Chandran K, Singhal N (2014) Clarifying the regulation of $\mathrm{NO} / \mathrm{N}_{2} \mathrm{O}$ production in Nitrosomonas europaea during anoxic-oxic transition via flux balance analysis of a metabolic network model. Water Res 60:267-277

Pipes WO, Gotaas HB (1960) Utilization of organic matter by Chlorella grown in sewage. Appl Microbiol 8:163-169

Pleissner D, Lam WC, Sun Z, Lin CSK (2013) Food waste as nutrient source in heterotrophic microalgae cultivation. Bioresour Technol 137:139-146

Pulz O (2001) Photobioreactors: production systems for phototrophic microorganisms. Appl Microbiol Biotechnol 57:287-293

Radakovits R, Jinkerson RE, Fuerstenberg SI, Tae H, Settlage RE, Boore JL, Posewitz MC (2012) Draft genome sequence and genetic transformation of the oleaginous alga Nannochloropsis gaditana. Nat Commun 3:686. doi:10.1038/ncomms 1688

Ratledge C, Wynn JP (2002) The biochemistry and molecular biology of lipid accumulation in oleaginous microorganisms. Adv Appl Microbiol 51:1-51

Ratledge C, Kanagachandran K, Anderson AJ, Grantham DJ, Stephenson JC (2001) Production of docosahexaenoic acid by Crypthecodinium cohnii grown in a $\mathrm{pH}$-auxostat culture with acetic acid as principal carbon source. Lipids 36:1241-1246

Raven JA (1976) The quantitative role of 'dark' respiratory processes in heterotrophic and photolithotrophic plant growth. Ann Bot 40:587-602

Rawat I, Ranjith Kumar R, Mutanda T, Bux F (2011) Dual role of microalgae: phycoremediation of domestic wastewater and biomass production for sustainable biofuels production. Appl Energy 88:3411-3424

Richmond A (1986) Cell response to environmental factors. In: Richmond A (ed) Handbook for microalgal mass culture. CRC Press, Boca Raton, pp 69-99 
Robertson DE, Jacobson SA, Morgan F, Berry D, Church GM, Afeyan NB (2011) A new dawn for industrial photosynthesis. Photosynth Res 107:269-277

Rodolfi L, Zittelli GC, Bassi N, Padovani G, Biondi N, Bonini G, Tredici MR (2009) Microalgae for oil: strain selection, induction of lipid synthesis and outdoor mass cultivation in a low-cost photobioreactor. Biotechnol Bioeng 102:100-112

Rosenberg JN, Kobayashi N, Barnes A, Noel EA, Betenbaugh MJ, Oyler GA (2014) Comparative analyses of three Chlorella species in response to light and sugar reveal distinctive lipid accumulation patterns in the microalga C. sorokiniana. PLoS One 9:e92460. doi:10.1371/journal. pone.009246

Sheehan J, Dunahay T, Benemann JR, Roessler P (1998) A look back a the U.S. Department of Energy's Aquatic Species Program-Biodiesel from algae. U.S. Department of Energy, Golden, CO. Report No. NREL/TP-580-24190

Shen Y, Yuan W, Pei Z, Mao E (2010) Heterotrophic culture of Chlorella protothecoides in various nitrogen sources for lipid production. Appl Biochem Biotechnol 160:1674-1684

Shi X, Jiang Y, Chen F (2002) High-yield production of lutein by the green microalga Chlorella protothecoides in heterotrophic fed-batch culture. Biotechnol Prog 18:723-727

Silaban A, Bai R, Gutierrez-Wing MT, Negulescu II, Rusch KA (2014) Effect of organic carbon, $\mathrm{C}: \mathrm{N}$ ratio and light on the growth and lipid productivity of microalgae/cyanobacteria coculture. Eng Life Sci 14:47-56

Solovchenko AE, Khozin-Goldberg I, Didi-Cohen S, Cohen Z, Merzlyak MN (2008) Effects of light intensity and nitrogen starvation on growth, total fatty acids and arachidonic acid in the green microalga Parietochloris incisa. J Appl Phycol 20:245-251

Subashchandrabose SR, Ramakrishnan B, Megharaj M, Venkateswarlu K, Naidu R (2013) Mixotrophic cyanobacteria and microalgae as distinctive biological agents for organic pollutant degradation. Environ Int 51:59-72

Tabernero A, Martín del Valle EM, Galán MA (2012) Evaluating the industrial potential of biodiesel from a microalgae heterotrophic culture: scale-up and economics. Biochem Eng J 63:104-115

Ueda R, Hirayama S, Sugata K, Nakayama H (1996) Process for the production of ethanol from microalgae. US Patent 5,578,472

Ueno Y, Kurano N, Miyachi S (1998) Ethanol production by dark fermentation in the marine green alga, Chlorococcum littorale. J Ferment Bioeng 86:38-43

Valderrama LT, Del Campo CM, Rodriguez CM, de-Bashan LE, Bashan Y (2002) Treatment of recalcitrant wastewater from ethanol and citric acid production using the microalga Chlorella vulgaris and the macrophyte Lemna minuscule. Water Res 36:4185-4192

Vanthoor-Koopmans M, Wijffels RH, Barbosa MJ, Eppink MHM (2013) Biorefinery of microalgae for food and fuel. Bioresour Technol 135:142-149

Vonshak A, Cheung SM, Chen F (2000) Mixotrophic growth modifies the response of Spirulina (Arthrospira) platensis (Cyanobacteria) cells to light. J Phycol 36:675-679

Wan M, Liu P, Xia J, Rosenberg JN, Oyler GA, Betenbaugh MJ, Nie Z, Qiu G (2011) The effect of mixotrophy on microalgal growth, lipid content, and expression levels of three pathway genes in Chlorella sorokiniana. Appl Microbiol Biotechnol 91:835-844

Wang Y, Chu J, Zhuang Y, Wang Y, Xia J, Zhang S (2009) Industrial bioprocess control and optimization in the context of systems biotechnology. Biotechnol Adv 27:989-995

Wang L, Li Y, Chen P, Min M, Chen Y, Zhu J, Ruan RR (2010) Anaerobic digested dairy manure as a nutrient supplement for cultivation of oil-rich green microalgae Chlorella sp. Bioresour Technol 101:2623-2628

Wang J, Yang H, Wang F (2014) Mixotrophic cultivation of microalgae for biodiesel production: status and prospects. Appl Biochem Biotechnol 172:3307-3329

Wijffels RH, Barbosa MJ, Eppink MHM (2010) Microalgae for the production of bulk chemicals and biofuels. Biofuels Bioprod Biorefin 4:287-295

Wijffels RH, Kruse O, Hellingwerf KJ (2013) Potential of industrial biotechnology with cyanobacteria and eukaryotic microalgae. Curr Opin Biotechnol 24:405-413 
Wiley PE, Harris L, Reinsch SS, Tozzi S (2013) Microalgae cultivation using offshore membrane enclosures for growing algae. J Sustain Bioenergy Syst 3:18-32

Wu Z, Shi X (2007) Optimization for high-density cultivation of heterotrophic Chlorella based on a hybrid neural network model. Lett Appl Microbiol 44:13-18

Xiong W, Li X, Xiang J, Wu Q (2008) High-density fermentation of microalga Chlorella protothecoides in bioreactor for microbio-diesel production. Appl Microbiol Biotechnol 78:29-36

Xiong W, Gao C, Yan D, Wu C, Wu Q (2010) Double $\mathrm{CO}_{2}$ fixation in photosynthesis-fermentation model enhances algal lipid synthesis for biodiesel production. Bioresour Technol 101:2287-2293

Xu H, Miao X, Wu Q (2006) High quality biodiesel production from a microalga Chlorella protothecoides by heterotrophic growth in fermenters. J Biotechnol 126:499-507

Yan D, Lu Y, Chen Y, Wu Q (2011) Waste molasses alone displaces glucose-based medium for microalgal fermentation towards cost-saving biodiesel production. Bioresour Technol 102:6487-6493

Yang C, Hua Q, Shimizu K (2000) Energetics and carbon metabolism during growth of microalgal cells under photoautotrophic, mixotrophic and cyclic light-autotrophic/dark-heterotrophic conditions. Biochem Eng J 6:87-102

Yang C, Hua Q, Shimizu K (2002) Integration of the information from gene expression and metabolic fluxes for the analysis of the regulatory mechanisms in Synechocystis. Appl Microbiol Biotechnol 58:813-822

Yen HW, Chang JT (2013) A two-stage cultivation process for the growth enhancement of Chlorella vulgaris. Bioprocess Biosyst Eng 36:1797-1801

Yu WL, Ansari W, Schoepp NG, Hannon MJ, Mayfield SP, Burkart MD (2011) Modifications of the metabolic pathways of lipid and triacylglycerol production in microalgae. Microb Cell Fact 10:91. doi:10.1186/1475-2859-10-91

Zhang X, Chen F, Johns MR (1999) Kinetic models for heterotrophic growth of Chlamydomonas reinhardtii in batch and fed-batch cultures. Process Biochem 35:385-389

Zhang TY, Wu YH, Zhu SF, Li FM, Hu HY (2013a) Isolation and heterotrophic cultivation of mixotrophic microalgae strains for domestic wastewater treatment and lipid production under dark condition. Bioresour Technol 149:586-589

Zhang XL, Yan S, Tyagi RD, Surampalli RY (2013b) Biodiesel production from heterotrophic microalgae through transesterification and nanotechnology application in the production. Renew Sustain Energy Rev 26:216-223

Zhang K, Sun B, She X, Zhao F, Cao Y, Ren D, Lu J (2014) Lipid production and composition of fatty acids in Chlorella vulgaris cultured using different methods: photoautotrophic, heterotrophic, and pure and mixed conditions. Ann Microbiol 64:1239-1246

Zheng Y, Chi Z, Lucker B, Chen S (2012) Two-stage heterotrophic and phototrophic culture strategy for algal biomass and lipid production. Bioresour Technol 103:484-488

Zhou Y, Schideman L, Yu G, Zhang Y (2013) A synergistic combination of algal wastewater treatment and hydrothermal biofuel production maximized by nutrient and carbon recycling. Energy Environ Sci 6:3765-3779 